Arkivoc

Free to Authors and Readers
A Platinum Open Access Journal for Organic Chemistry

Paper

DOAJ Seal
Arkivoc 2021, part iv, 206-229

\title{
Total Synthesis of the Chlorinated Indigo-N-Glycosides Akashin A, B and C
}

\author{
Alexander T. Pfretzschner and Carlo Unverzagt* \\ University of Bayreuth, Bioorganic Chemistry, Universitätsstraße 30, 95447 Bayreuth, Germany \\ Email: carlo.unverzagt@uni-bayreuth.de
}

Received 09-23-2020

Accepted 11-06-2020

Published on line 12-05-2020

\section{Abstract}

A total synthesis of the indigo-derived natural products akashin A-C was developed. The key step was the $N$ glycosylation of soluble $N$-benzylated-indigos with a suitably protected viosaminyl trichloroacetimidate. This donor was obtained from D-galactose. Unusual intermediates in the glycosylation reaction of $N$-benzylindigo (O-glycosides) were observed. The final transformations yielding akashin A required the adjustment of the reaction conditions to preserve the sensitive chlorinated indigo scaffold. Akashin A was converted to akashin $B$ and $C$, all of which display an unusually high dextrarotatory optical rotation thus establishing the Dconfiguration in the sugar part of the natural akashins $A-C$.

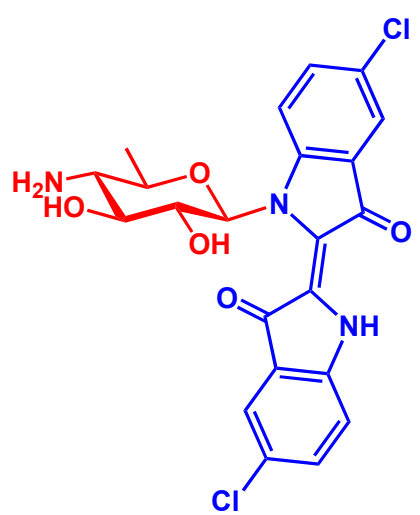

Akashin A

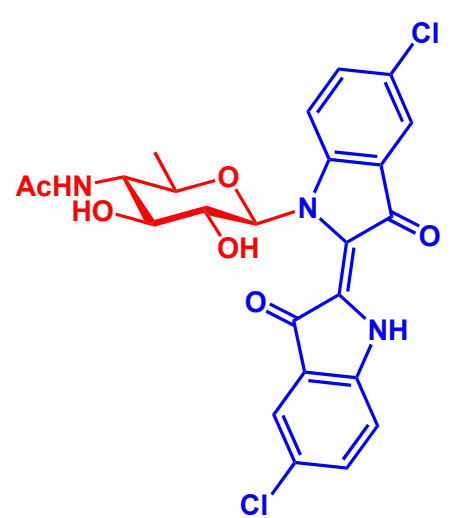

Akashin B

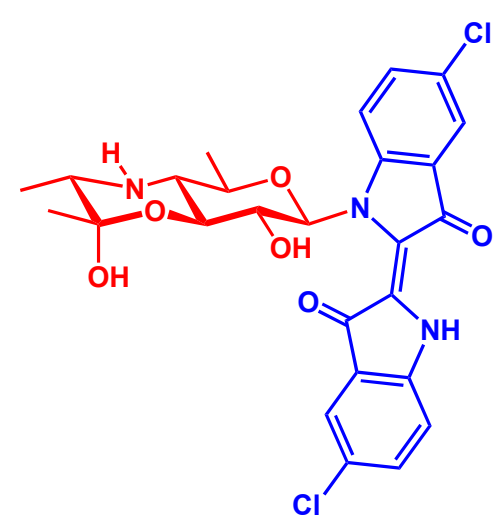

Akashin C

Keywords: Indigo, $\mathrm{N}$-glycoside, glycosylation, protecting groups, natural products 


\section{Introduction}

Heterocyclic $N$-glycosides are part of many natural products with pharmacological relevance. For example, the $\mathrm{N}$-glyosylated natural products rebeccamycin, UCN-01, staurosporine and derivatives, all containing bisindole aglycons, ${ }^{1-6}$ exhibit a strong cytotoxic activity. In the case of an $\mathrm{N}$-glycosylated derivate of isoindigo named NATURA $^{7-8}$ and of $N$-( $\beta$-D-rhamnosyl)indirubin ${ }^{9-10}$ anti-proliferative activities were found. No bioactive analogues of indigo were known until 2002, when Laatsch et al. ${ }^{11-12}$ reported the isolation of akashin A (1), B (2) and C (3) from terrestric Streptomyces sp. GW 48/1497 (Figure 1). The akashins are colourful dyes with unusually high optical rotations. In contrast to pharmacologically inactive indigo the akashins show a remarkable cancerostatic activity against various human cancer cell lines. Akashin A was recently also discovered in the marine derived Streptomyces sp. strain F001. ${ }^{13}$

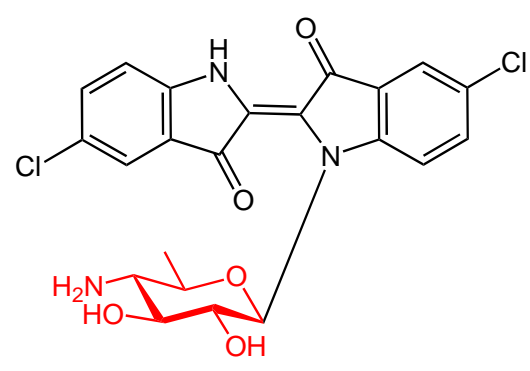

akashin A (1)

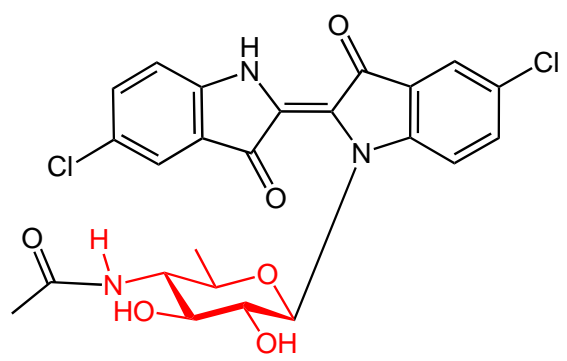

akashin B (2)

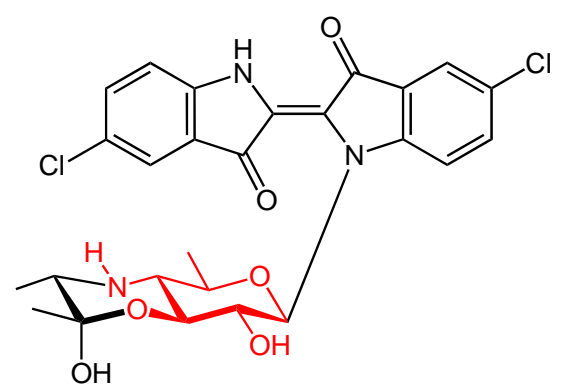

akashin C (3)

Scheme 1. The akashins A-C are natural products from Streptomyces containing a dichloroindigo aglycon $N$ glycosidically linked to the rare deoxy sugar viosamine (red).

The akashins A-C were reported to be composed of dichloroindigo $\mathrm{N}$-glycosidically linked to 4-amino-4,6dideoxy-glucopyranose (viosamine) ${ }^{14}$ with a hitherto undetermined absolute configuration. This rare carbohydrate is present as D-viosamine in the tetrasaccharide antigen of the Bacillius anthracis exosporium glycoprotein $\mathrm{Bcl} .{ }^{15}$ Additionally, also the enantiomer L-viosamine is part of natural products and antibiotics like amicetin, ${ }^{16}$ norplicacetin, ${ }^{17}$ tallysomicin ${ }^{18}$ and the family of calicheamicins. ${ }^{19}$

Several approaches were developed for the synthesis of $N$-glycosylated indole derivatives comprising the indole-indolin-method by Preobrazhenskaya ${ }^{[13]}$ and the direct acid-mediated glycosylation of substituted indoles $^{[14]}$. For more than 100 years the preparation of synthetic indigo is known by dimerization of indoxyl in the presence of oxygen ${ }^{[15]}$. In analogy, a conceivable synthesis of akashins might be accomplished by the $N$ glycosylation of a chlorinated 3-indolyl acetate with suitable viosaminyldonor followed by a heterodimerization with chlorinated 3-indolyl acetate. Furthermore, a direct glycosylation of indigo or its derivatives could also be considered. Since the solubility of indigos is very low the use of derivatives for glycosylation has practical advantages. In particular $\mathrm{N}$-benzylindigo ${ }^{20}$ and also dehydroindigo ${ }^{21}$ were shown by the Langer group to serve as suitable acceptors in glycosylation reactions.

Here we report the first total synthesis of akashin A and its subsequent derivatization to akashin B and C. 


\section{Results and Discussion}

After a number of unsuccessful attempts to obtain soluble derivatives of indigo for glycosylation reactions we adopted the strategy developed by Langer ${ }^{21}$ using $N$-benzylindigo 6 in combination with glycosyltrichloroacetimidates. The benzyl group of the corresponding indigo $\mathrm{N}$-glycosides can be removed under oxidative conditions. However the formation of orthoester-like amide acetals in the glycosylation of 6 required the use of pivaloyl groups ${ }^{22}$ in the glycosyl donor, which could not be removed without affecting the indigo scaffold. ${ }^{21}$ As a model compound to demonstrate the $\mathrm{N}$-glycosylation of $\mathrm{N}$-benzylindigo 6 and the subsequent complete deprotection we selected the indigo glucoside 4 (Figure 2).

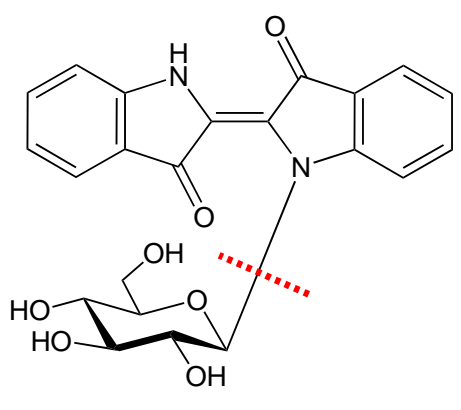

4

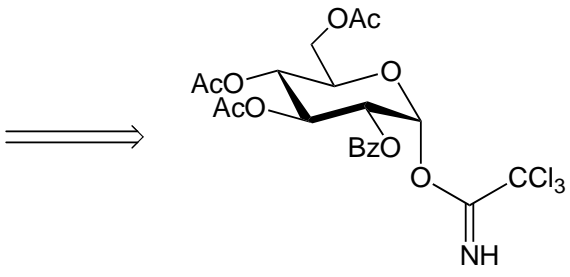

5

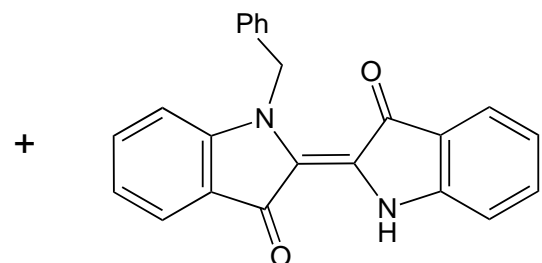

6

Scheme 2. Retrosynthesis of $N$ - $\beta$-D-glucopyranosyl-indigo 4 as a model compound.

In view of the previous synthesis of tryptophan $\mathrm{N}$-glucoside ${ }^{23}$ we chose donor $\mathbf{5}$ with a benzoyl group at O2. In analogy to 2-pivaloyl groups a benzoate should also reduce the amount of orthoester-like side products during glycosylation and permit cleavage under relatively mild conditions. ${ }^{23}$

The required trichloroacetimidate ${ }^{24}$ building block 5 (Figure 3) was synthesized in three steps from 1,3,4,6tetraacetyl-glucose $7 .{ }^{25}$ After benzoylation of $\mathrm{OH}-2$ the anomeric acetate of compound 7 a was cleaved with hydrazine acetate and the intermediate hemiacetal $\mathbf{7 b}$ was converted to the imidate $\mathbf{5}$ using DBU and trichloroacetonitrile in an overall yield of 75\%. $N$-Benzylindigo 6 was obtained from indigo 8 in $48 \%$ yield accompanied by only small amounts of the green by-product $N, N^{\prime}$-dibenzylindigo.

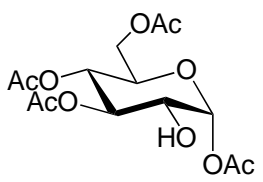

7

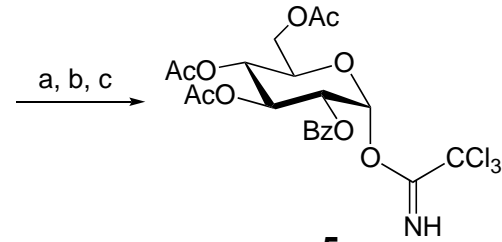

5

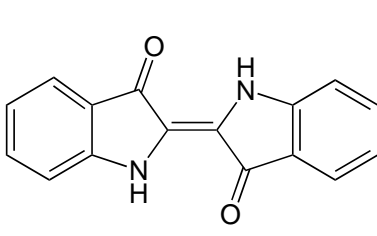

8
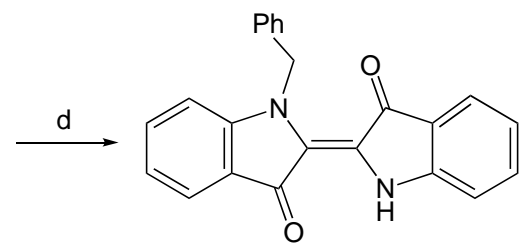

6

Scheme 3. Synthesis of 5 and 6 . Reagents and conditions: a) $\mathrm{BzCl}$, pyridine, $\mathrm{CH}_{2} \mathrm{Cl}_{2}$ (99\%); b) hydrazine acetate, $\operatorname{DMF}(80 \%)$; c) $\mathrm{Cl}_{3} \mathrm{CCN}, \mathrm{DBU}, \mathrm{CH}_{2} \mathrm{Cl}_{2}, 0^{\circ} \mathrm{C}(90 \%)$; d) $\mathrm{NaH}, \mathrm{DMF}, \mathrm{BnBr}(48 \%)$.

The glycosylation of $\mathrm{N}$-benzylindigo 6 with the benzoylated donor 5 using TMSOTf furnished the desired indigo- $N$-glucoside 9 in 23\% yield (Figure 4). Notably, the reaction proceeded in two steps accompanied by a colour change of the product spots on TLC. At low temperatures a red compound formed initially, which disappeared at higher temperatures and led to a new turquoise spot. As proposed by Langer ${ }^{20}$ an $O$-glycoside 
(9a, red) appeared first followed by rearrangement to the stable $N$-glycoside (9, turquoise). Variation of the glycosylation conditions did not increase the yield significantly. However, some of the starting material $\mathbf{6}$ could be recovered. The oxidative removal of the benzyl group was investigated next. Using a heated mixture of toluene/HOAc (1:1) in the presence of air led to the slow debenzylation of 9 over 12-18h and furnished the debenzylated intermediate $\mathbf{9 b}$ (see supporting information) in a yield of $61 \%$. Subsequently, the ester groups of 9a were removed using dilute $\mathrm{NaOMe} / \mathrm{MeOH}$ ( $\mathrm{c}=0.01 \mathrm{~mol} / \mathrm{l}$ ) furnishing the desired $\mathrm{N}$-glycoside 4 in $51 \%$ yield. Higher concentrations of base or elevated temperatures resulted in the loss of the blue colour of the indigo core and led to inferior yields.

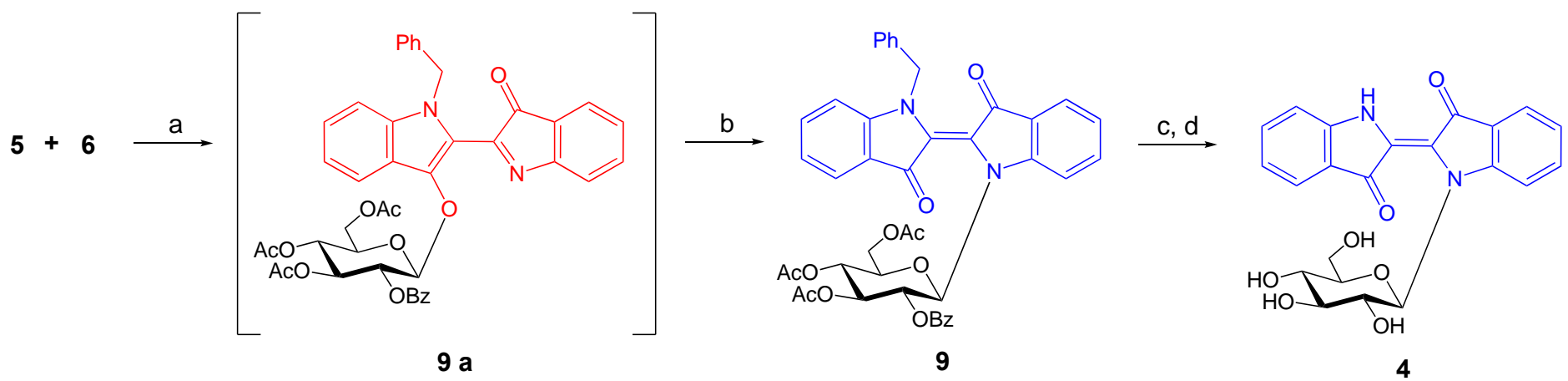

Scheme 4. Synthesis of $\mathrm{N}$-glucosyl indigo 4. Reagents and conditions: a) TMSOTf, $4 \AA \mathrm{AS}, \mathrm{CH}_{2} \mathrm{Cl}_{2},-18^{\circ} \mathrm{C}, 3 \mathrm{~h}$; b) TMSOTf, $4 \AA$ MS, $\mathrm{CH}_{2} \mathrm{Cl}_{2}$, RT, 10-12 h (23\% over two steps); c) toluene/HOAc (1:1), $50{ }^{\circ} \mathrm{C}$, air, $12-18 \mathrm{~h},(61 \%) ; \mathrm{d}$ ) i. $\mathrm{NaOMe} / \mathrm{MeOH}, 11 \mathrm{~h}$; ii. Amberlyst $15\left(\mathrm{H}^{+}\right),(51 \%)$.

Following the synthetic route to $\mathrm{N}$-glucosylated indigo 4 the synthesis of akashin A was planned based on the building blocks $\mathbf{1 0}$ and $\mathbf{1 1}$. The viosaminyldonor $\mathbf{1 0}$ was conceived to contain benzoates, an azido group as a precursor for the free amine and an activation as a trichloroacetimidate. As an acceptor the benzylated 5,5'dichloroindigo 11 was considered. The other akashins B (2) and C (3) should be accessible by chemical transformation of akashin A 1 (Scheme 5).

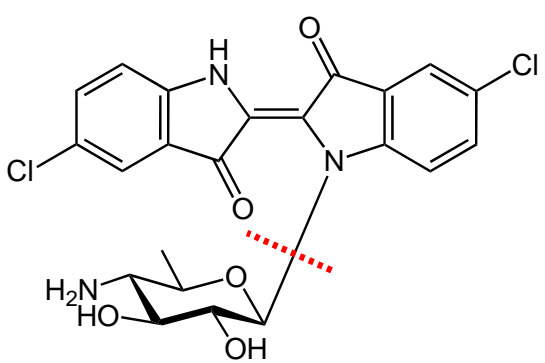

akashin $\mathrm{A}(\mathbf{1})$
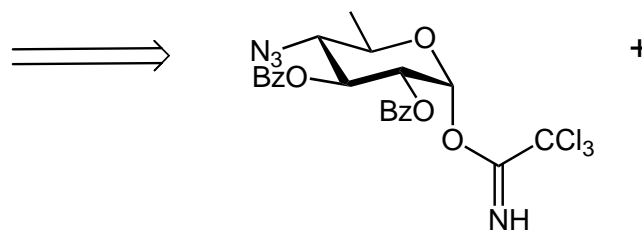

10<smiles>O=C1C(=C2C(=O)c3cc(Cl)ccc3N2Cc2ccccc2)Nc2ccc(Cl)cc21</smiles>

11

Scheme 5. Retrosynthesis of akashin A.

The synthesis of the D-gluco-configurated viosaminyldonor 10 required the introduction of an equatorial azide and deoxygenation of $\mathrm{OH}-6$ when starting from D-galactose (Figure 6). After 4,6-benzylidenation of Dgalactose and subsequent perbenzoylation it was found that the reactivity of tribenzoyl-4,6-benzylidene-Dgalactose $12^{26}$ in the following reactions was depending on the anomeric configuration. Thus, both anomers 
were separated by flash chromatography $(12 \alpha: 12 \beta=87: 13)$ and individual syntheses were carried out with each anomer.

After acidic removal of the benzylidene group $(12 \alpha-1,12 \beta-1$ the liberated primary alcohol was selectively converted to a halide. Reduction of the bromides using various methods was not successful. However, catalytic hydrogenation of the primary iodide $13 \alpha$ gave fucoside $14 \alpha$ in yields of up to $82 \%$ depending on the reaction volume. In contrast, the maximum yield of the corresponding beta anomer $14 \beta$ was only $35 \%$. By recycling the recoverable starting material $\mathbf{1 3 \beta}$ and the Pd-catalyst the overall yield could be improved. The equatorial azido function was introduced efficiently by formation of a triflate and subsequent nucleophilic substitution at C-4 using sodium azide in DMF. Both azides $15 \alpha, \beta$ were selectively deprotected at the anomeric position with hydrazine hydrate (10a) and converted to the desired viosaminyl-trichloroacetimidate $10 .{ }^{27}$ The overall yields (9 steps) were $33 \%$ via the $\alpha$-anomer $12 \alpha$ vs. $17 \%$ from the $\beta$-anomer $\mathbf{1 2} \beta$.

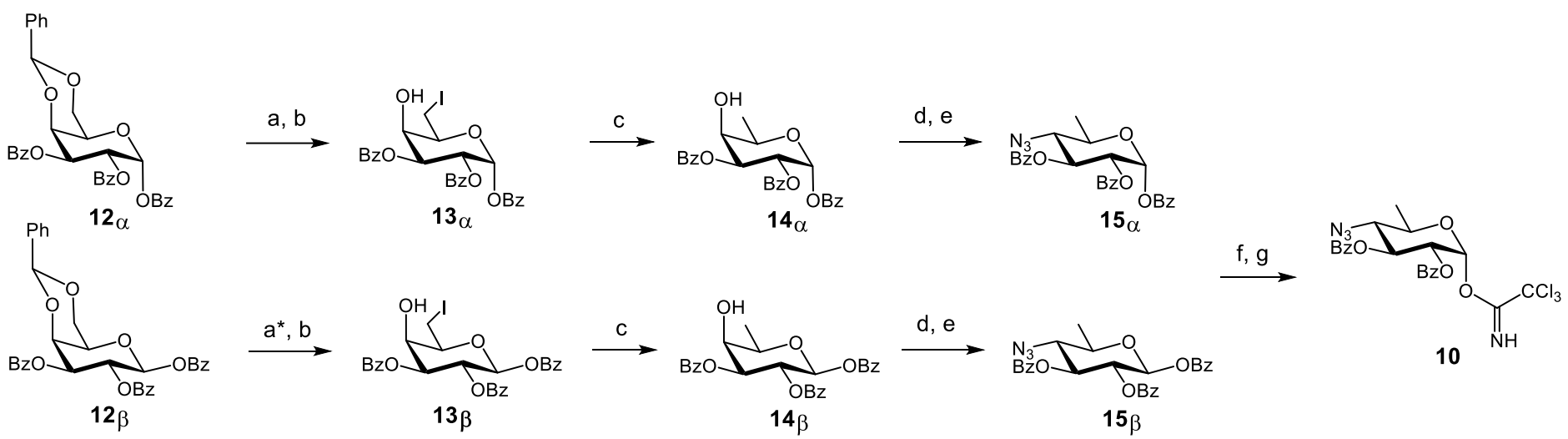

Scheme 6. Synthesis of donor 10. Reagents and conditions: a) p- $\mathrm{TsOH}-\mathrm{H}_{2} \mathrm{O}$, acetonitrile, $\left.\mathrm{MeOH}, 1 \mathrm{~d}(74 \%) ; a^{*}\right)$ $2 \mathrm{~N} \mathrm{HCl}$, acetonitrile, $\mathrm{MeOH}, 80^{\circ} \mathrm{C}, 2 \mathrm{~h}(81 \%) ;$ b) $\left.\mathrm{NIS}, \mathrm{PPh}_{3}, \mathrm{DMF}, 0-50{ }^{\circ} \mathrm{C}, 1 \mathrm{~d}, 13 \alpha(73 \%), 13 \beta(85 \%) ; \mathrm{c}\right) \mathrm{H}, \mathrm{Pd} / \mathrm{C}$ (10\%), MeOH/HOAc (10:1), $14 \alpha$ (56-83\%), $14 \beta$ (35\%); d) $\mathrm{Tf}_{2} \mathrm{O}$, pyridine, $\mathrm{CH}_{2} \mathrm{Cl}_{2},-18{ }^{\circ} \mathrm{C}, 2 \mathrm{~h}$; e) $\mathrm{NaN}_{3}, \mathrm{DMF}, 30$ $\min , 15 \alpha$ (92\%), $15 \beta$ (84\%) over two steps; f) hydrazine acetate, DMF, RT, 3-6 h; g) $\mathrm{Cl}_{3} \mathrm{CCN}, \mathrm{DBU}, \mathrm{CH}_{2} \mathrm{Cl}_{2}, 0{ }^{\circ} \mathrm{C}, 2$ h, (95\% over two steps).

5,5'-Dichloroindigo $19^{28}$ was generated by the classic method via oxidative dimerization of indoxyl to indigo in basic solution (Figure 7). ${ }^{29}$ The required 3-acetoxy-5-chloroindole $18^{30}$ was prepared by reacting 4chloroanthranilic acid 16 and chloroacetic acid to 4-chlorophenylglycine-2-carboxylic acid 17. Subsequent ring closure was effected by acetic anhydride giving 1,3-diacetyl-5-chloroindole. The cleavage of both acetyl groups in $\mathrm{NaOH}$ under argon followed by selective O-acetylation of the intermediate indoxyl provided 18 in $67 \%$ yield over two steps. The dichloroindigo 19 (73\%) was obtained by oxidative dimerization of 18 under basic conditions. Due to the low solubility of 19 NMR analysis was carried out after reduction ${ }^{31}$ to the corresponding vat indigo 19v. N-Benzylation of 19 gave the selectively protected dichloroindigo derivative $1159 \%$. 


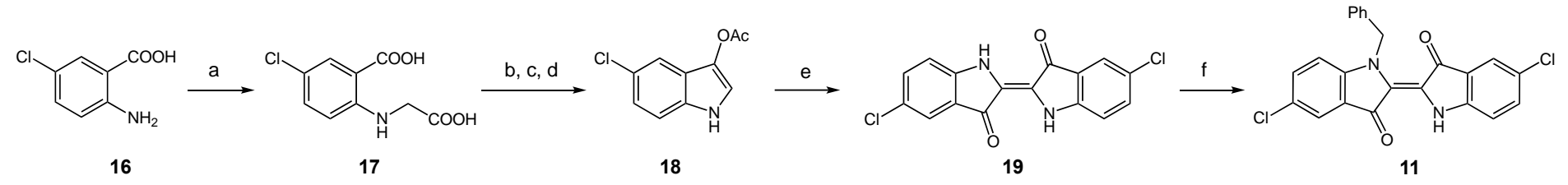

Scheme 7. Synthesis of $\mathrm{N}$-benzyl-5,5'-dichloroindigo 11. Reagents and conditions: a) chloroacetic acid, $\mathrm{Na}_{2} \mathrm{CO}_{3}$, reflux, $6 \mathrm{~h}$ (66\%); b) $\mathrm{Ac}_{2} \mathrm{O}, \mathrm{NaOAc}$, reflux, $\left.3 \mathrm{~h},(63 \%) ; \mathrm{c}\right) \mathrm{NaOH}$, reflux, argon, $30 \mathrm{~min}$; d) $\mathrm{Ac}_{2} \mathrm{O}, 0^{\circ} \mathrm{C}, \operatorname{argon}, 45$ min, (67\% over two steps); e) $\mathrm{NaOH}, \mathrm{MeOH}$, air, (73\%); f) NaH, DMF, BnBr, 2 h, (59\%).

$\mathrm{N}$-benzyl-5,5'-dichloroindigo $\mathbf{1 1}$ was subsequently reacted with imidate $\mathbf{1 0}$ in the presence of TMSOTf as an activator (Scheme 8). By using less concentrated reaction conditions (18 $\mathrm{mM} 10$ and 11) the yield of the indigo- $N$-glycoside 20 was raised to 45\%. Unreacted $N$-benzylindigo 11 (54\%) was recovered and reused. The oxidative removal of the $\mathrm{N}$-benzyl group of 20 proceeded smoothly over a period of $18 \mathrm{~h}$. After purification by flash chromatography the indigo glycoside $\mathbf{2 1}$ was obtained in a yield of $60 \%$.

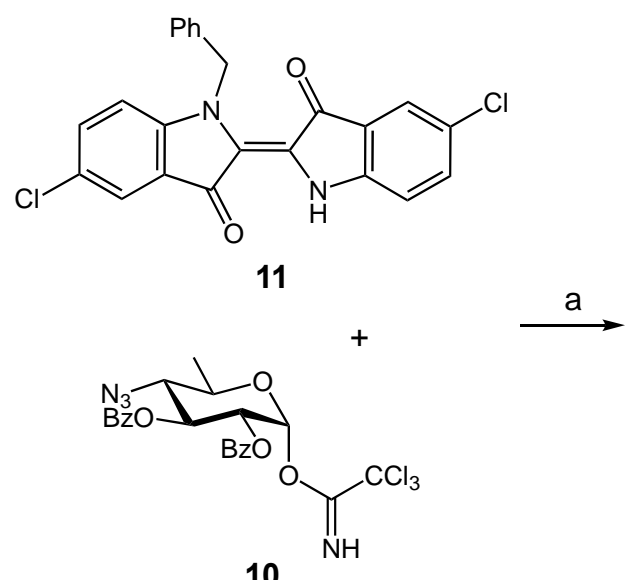

10

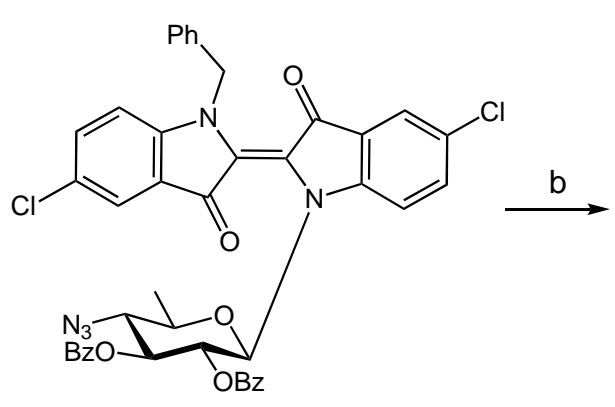

20

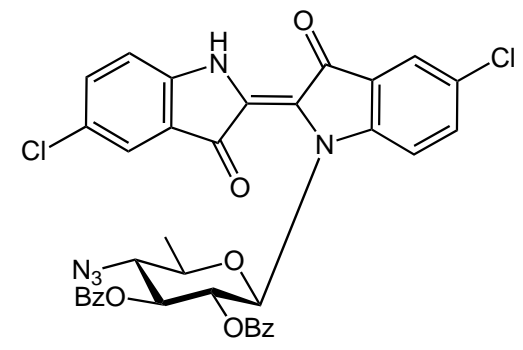

21

Scheme 8. Synthesis of the dichloroindigo- $N$-glycoside 21. Reagents and conditions: a) TMSOTf, $4 \AA$ MS, $\mathrm{CH}_{2} \mathrm{Cl}_{2},-18{ }^{\circ} \mathrm{C}$ to RT (15 h), (45\%); b) toluene/HOAC (1:1), $60{ }^{\circ} \mathrm{C}$, air, $12-18 \mathrm{~h},(60 \%)$.

As observed before in the synthesis of 4 (Scheme 4 ) the $N$-glycosylation of the $N$-benzylated dichloroindigo 11 initially also gave a red intermediate at low temperatures (Scheme 9). Upon warming this intermediate disappeared and was converted to the $\mathbf{N}$-glycoside $\mathbf{2 0}$ visible as a turquoise spot on TLC. We decided to isolate the red intermediate and determine its structure. Thus, a glycosylation reaction using 10 and 11 was kept at $-18{ }^{\circ} \mathrm{C}$ and quenched with $\mathrm{Et}_{3} \mathrm{~N}$ after $2 \mathrm{~h}$. Under slightly basic conditions the red intermediate 20a was sufficiently stable for flash chromatography in the presence of $\mathrm{Et}_{3} \mathrm{~N}$ added to the solvents. In the course of this purification a second red compound (20b) was isolated, which nearly comigrated with the turquoise spot of the $\mathrm{N}$-glycoside $\mathbf{2 1}$ on TLC. 


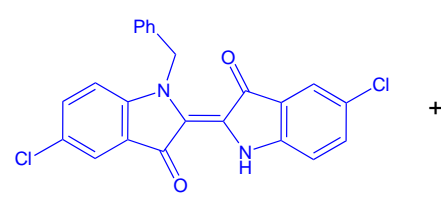

11

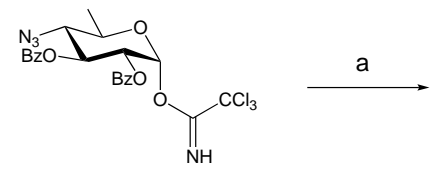

10

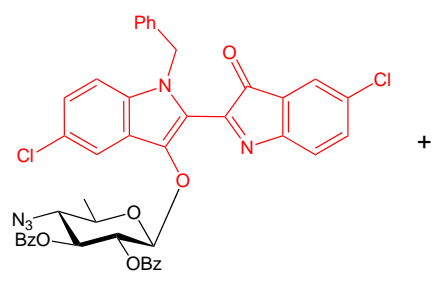

$20 a$

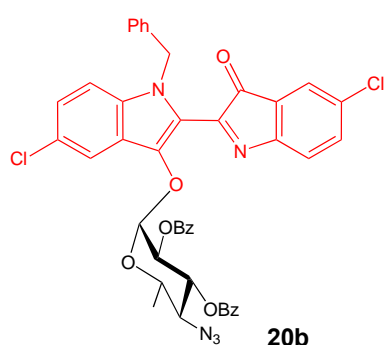

Scheme 9. Synthesis of the reaction intermediates 20a ( $\beta$-O-glycoside) and 20b ( $\alpha$-O-glycoside). Reagents and conditions: a) TMSOTf, $4 \AA \mathrm{MS}, \mathrm{CH}_{2} \mathrm{Cl}_{2},-18^{\circ} \mathrm{C}, 2 \mathrm{~h}$.

The structures of the red indigo glycosides were determined by a set of 2D-NMR experiments (HH-COSY, HMQC, HMQC-COSY, NOESY, HMBC). ${ }^{32}$ In the HMBC spectrum of 20a (Scheme 10) the most relevant cross peaks were correlating $\mathrm{C}-3$ ' with the anomeric proton $\mathrm{H}-1$ " and $\mathrm{C}-2$ ' with the benzylic $\mathrm{H}-8$ ' thus localizing the glycoside at the oxygen of the benzylated half of the indigo. A strong NOESY cross peak was correlating $\mathrm{H}-8$ 'a with $\mathrm{H}-\mathbf{7}^{\prime}$. The $\mathrm{O}$-glycoside was further supported by the ${ }^{13} \mathrm{C}-\mathrm{NMR}$ signal for the anomeric carbon $\mathrm{C}-1^{11}$ of $21 \mathrm{a}$ located at $101.4 \mathrm{ppm}$ ( $\mathrm{N}$-glycoside 20: $\mathrm{C}-1^{\prime \prime}: 85.3 \mathrm{ppm}$ ). The $\beta$-linkages of 20a and 20 were deduced from the large coupling constants for $\mathrm{H}-1^{\prime \prime}$ of $20 \mathrm{a}\left(J_{1^{\prime \prime}, 2^{\prime \prime}}=8.0 \mathrm{~Hz}\right.$ at $\left.5.3 \mathrm{ppm}\right)$ and $\mathrm{H}-1^{\prime \prime}$ of $20\left(J_{1^{\prime \prime}, 2^{\prime \prime}}=9.1 \mathrm{~Hz}\right.$ at $\left.6.0 \mathrm{ppm}\right)$. The NMR results showed that 20a is an $O$-glycoside thus confirming the mechanistic proposal that indigo $O$ glycosides occur as intermediates in the synthesis of benzylated indigo $\mathrm{N}$-glycosides. ${ }^{20}$

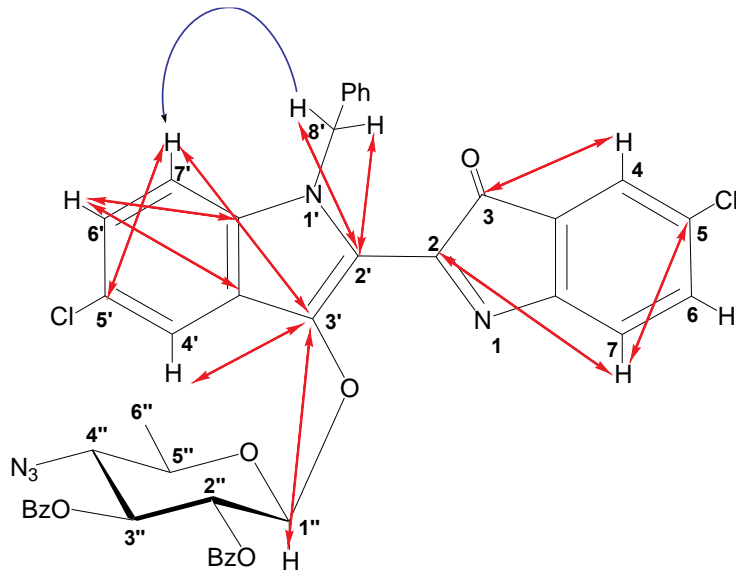

$20 \mathrm{a}$

Scheme 10. HMBC (red)- and NOESY (blue)-correlations found for the red O-glycosidically linked indigo intermediate 20a.

The ${ }^{1} \mathrm{H}-\mathrm{NMR}$ spectra of $\mathbf{2 0 \mathrm { b }}$ also indicated an $\mathrm{O}$-glycoside $\left({ }^{13} \mathrm{C}-\mathrm{NMR}\right.$ signal at $99.3 \mathrm{ppm}$ for $\left.\mathrm{C}-\mathrm{1}^{\prime \prime}\right)$, however, with a small anomeric coupling constant $\left(J_{1 ", 2 "}=3.4 \mathrm{~Hz}\right.$ for $\left.\mathrm{H}-1^{\prime \prime}\right)$ corresponding to an $\alpha$-glycoside. We first assumed an orthoester but in the ${ }^{13} \mathrm{C}-\mathrm{NMR}$ spectrum of $20 \mathrm{~b}$ the carbonyl groups of both benzoates were present. Furthermore, the chemical shift of H-2 in 20b was very similar to 20a (20a H-2": 5.38 ppm: 20b H-2": $5,35 \mathrm{ppm}$ ) thus ruling out an orthoester structure for $\mathbf{2 0 b}$.

The final steps in the synthesis of akashin A required the conversion of the azido function of $\mathbf{2 1}$ to a primary amine and the removal of the benzoates (Scheme 11). We found that both steps could be performed simultaneously using propanedithiol as a reductant under basic conditions. For best results thoroughly 
degassed and dried solvents were used under an argon atmosphere. When adding propanedithiol ${ }^{33}$ to a methanolic solution of $\mathbf{2 1}$ containing DIPEA the blue colour of the starting material vanished immediately. The pale yellow colour of the solution indicated the formation of a leukoindigo. Under these conditions the combined reduction of the azide and the debenzoylation took about $4 \mathrm{~d}$ until completion. At this point reoxidation of the indigo was initiated by acidification with $1 \%$ HOAc in dichloromethane accompanied by vigorous stirring of the reaction mixture in an open flask. The entire reaction sequence needed to be carried out under strict exclusion of light to prevent extensive radical dehalogenation of the reduced dichloroindigo. ${ }^{31}$ In the presence of light the corresponding indigo $\mathrm{N}$-glycosides with only one chloroatom or completely dehalogenated indigo were formed. After purification by solid phase extraction (C18) and size exclusion chromatography the intensely blue coloured akashin A (1) was isolated in yields up to $73 \%$.

The selective $\mathrm{N}$-acetylation of $\mathbf{1}$ was performed using a mixture of acetic anhydride and water ${ }^{34}$ in methanol (Scheme 11). After purification by solid phase extraction akashin B (2) was obtained in nearly quantitative yield. The conversion of 1 to akashin C (3) was achieved by condensing diacetyl ${ }^{35}$ (butane-2,3dione) with 1 to a cyclic imine/hemiketal intermediate followed by a selective reduction of the imine with $\mathrm{NaCNBH}_{3}$. Both steps were carried out in a one-pot procedure and gave akashin $\mathrm{C}(3)$ in $59 \%$ yield after flash chromatography. The axial orientation of the $\mathrm{OH}$-group of the cyclic hemiketal in the final product $\mathbf{3}$ is stabilized by the anomeric effect. In the reductive amination step the formation of the sterically less hindered equatorial methyl group is favored.

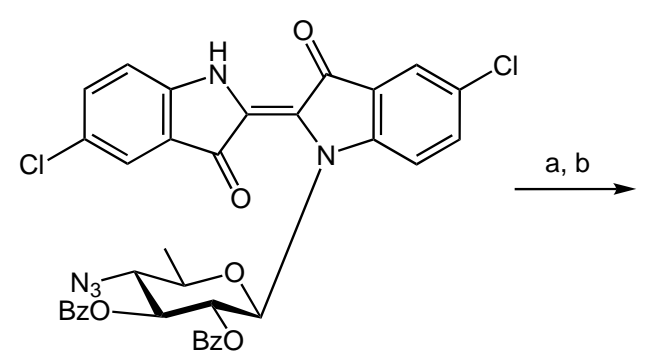

21

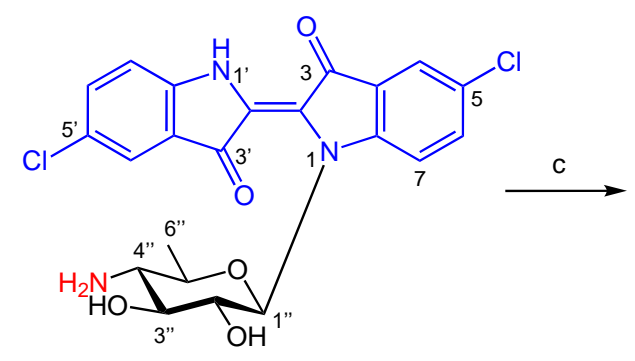

akashin $\mathrm{A}(\mathbf{1})$

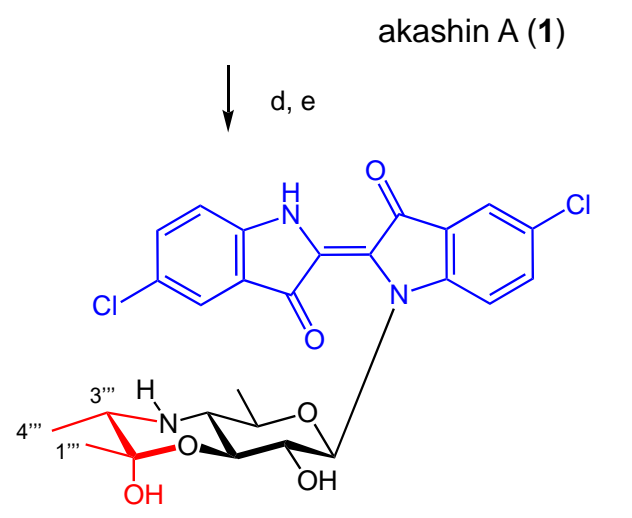

akashin C (3)

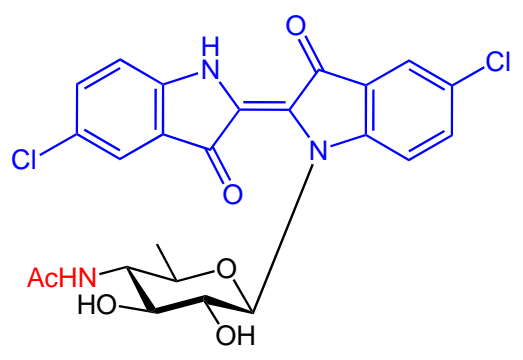

akashin B (2)

akashin

Scheme 11. Final steps in the synthesis of akashin A, B and C. Reagents and conditions: a) propanedithiol, DIPEA, MeOH, exclusion of light, 3-4 d, argon; b) $\mathrm{CH}_{2} \mathrm{Cl}_{2} / \mathrm{AcOH}$ (99:1), air, 60-90 min, (39-73\% over two steps); c) $\mathrm{Ac}_{2} \mathrm{O}, \mathrm{MeOH}, \mathrm{H}_{2} \mathrm{O}, 30-90 \mathrm{~min}$, (quantitative); d) diacetyl, camphorsulfonic acid (CSA), trimethylorthoformate, $\mathrm{MeOH}, \mathrm{RT}, 2-3 \mathrm{~h}$; e) $\mathrm{NaCNBH}_{3} ; \mathrm{MeOH}, \mathrm{RT}, 20-30$ min, (59\% over two steps).

The NMR signals of the synthetic akashins A-C (1-3) were assigned by an extensive set of NMR experiments $\left({ }^{1} \mathrm{H}, \mathrm{JMOD},(\mathrm{HH}-\mathrm{COSY}, \mathrm{HMQC}, \mathrm{HMQC}-\mathrm{COSY})\right.$ and compared with the data reported for the 
isolated natural compounds. ${ }^{11}$ The assignment of the resonances was in line with the data reported previously. Only minor differences were found for the neutral akashin B (2) where the position of the reported ${ }^{1} \mathrm{H}$-signals were deviating only up to $\left(\Delta \delta^{\max }\right) 0.06 \mathrm{ppm}$ and the ${ }^{13} \mathrm{C}$ signals up to $0.7 \mathrm{ppm}$ in $\mathrm{DMSO}-\mathrm{D}_{6}$. The chemical shifts of akashin A (1) containing a primary amino group were less consistent with the reported data of the ${ }^{1} \mathrm{H}$-spectra $\left(\Delta \delta^{\max }=-0.5 \mathrm{ppm}\right.$ for $\mathrm{H}-4^{\prime \prime}$ of 1$)$ and the ${ }^{13} \mathrm{C}$-spectra $\left(\Delta \delta^{\max }=+4.2 \mathrm{ppm}\right.$ for $\mathrm{C}-5^{\prime \prime}$ of 1$)$. In contrast, for akashin $\mathrm{C}(3)$ with a secondary amine the comparison of the NMR data was quite satisfactory for protons $\left({ }^{1} \mathrm{H}: \Delta \delta^{\max }=-0.15 \mathrm{ppm}\right.$ for $\mathrm{H}-3^{\prime \prime}$ of 3$)$ and ${ }^{13} \mathrm{C}(\Delta \delta=-1.7 \pm 0.4 \mathrm{ppm}$ for all signals of 3$)$. The ${ }^{1} \mathrm{H}-\mathrm{NMR}$ shifts of the sugar part of 1 were significantly affected by adding some hydrochloric acid to the NMR sample of synthetic 1 . Under these conditions the deviation $(\Delta \delta)$ of the most affected sugar signal $(\mathrm{H}-4$ " of 1$)$ was lowered from $-0.5 \mathrm{ppm}$ to $+0.11 \mathrm{ppm}$. The higher similarity of the ${ }^{1} \mathrm{H}-\mathrm{NMR}$ spectra of isolated akashin $\mathrm{A}$ and synthetic akashin $\mathrm{A}(\mathbf{1})+\mathrm{HCl}$ indicated that the isolated natural product $\mathbf{1}$ was presumably measured as an ammonium salt.

The isolated akashins A-C have in common an unusually high specific optical rotation with values of several thousand degrees $\left(+2560^{\circ}\right.$ to $\left.+3100^{\circ}\right)$ requiring the measurement at very low concentrations $(\sim 10$ $\mu \mathrm{g} / \mathrm{mL})$. We also found very high positive values $\left(+1143^{\circ}\right.$ to $\left.+4138^{\circ}\right)$ for the synthetic akashins A-C (1-3). It can be assumed that the chiral properties of the akashins are depending on the stereochemistry of the sugar part. Vice versa the very high positive optical rotations of both the synthetic and the isolated akashins indicate that the absolute configuration of the sugar moiety is identical. Thus, the viosamine part of the natural akashins is D-configurated.

\section{Conclusions}

In conclusion we have developed a short synthesis for the natural dichloroindigo $\mathrm{N}$-glycosides akashin A-C. The key building blocks were a benzyl-protected dichloroindigo and a suitably protected D-viosaminyl donor. Surprisingly an $\alpha$ - and a $\beta$-linked O-glycoside were isolated as intermediates of the $N$-glycosylation reaction. The identity of the synthetic and the isolated akashins was confirmed by NMR. Furthermore, the Dconfiguration of the dideoxy sugar viosamine in the natural products was established by optical rotation.

\section{Experimental Section}

General. Unless otherwise noted, chemicals were obtained from commercial suppliers and used without further purification. Methanol was dried with Mg-turnings ( $5 \mathrm{~g} / \mathrm{L}$ ) and distilled under argon. Dichloromethane was dried over phosphorous pentoxide, distilled and kept over $3 \AA$ molecular sieves. Dimethylformamide and pyridine were p.a. quality, acetonitrile was HPLC grade (>99.9\%). TLC was performed on precoated silica gel plates (Macherey-Nagel, Alugram Sil G/UV254, silica layer thickness: $0.25 \mathrm{~mm}$ ). Fluorescent compounds were detected using a UV illuminator (254 nm). Glycosylated compounds were detected using a 1:1 solution of $0.2 \%$ of resorcinol monomethyl ether (3-methoxyphenol) and $2 \mathrm{M}$ sulfuric acid in ethanol followed by heating. Flash chromatography was carried out on Merck silica gel (0.040-0.063 mm). For solid phase extraction Waters SepPak Vac C18 cartridges were used. Optical rotations were determined in $1 \mathrm{dm}$ cells ( $1 \mathrm{~mL}$ ) in a Perkin-Elmer 241 polarimeter at $589 \mathrm{~nm}$. UV measurements were performed with a Specord 200 Jena Analytik spectrophotometer. ESI-mass spectra were recorded on a Micromass LCT ESI-TOF mass spectrometer coupled to an Agilent HP 1100 HPLC with a photodiode array UV detector. The following reversed phase columns were 
used for LC-MS: YMC-Pack Pro C8, S-3 $\mu \mathrm{m}(50 \times 2.1 \mathrm{~mm})$, YMC-Pack Pro C18, S-3 $\mu \mathrm{m}(50 \times 2.1 \mathrm{~mm})$. Eluent A, water $(0.1 \%$ formic acid); eluent $B$, acetonitrile $(0.1 \%$ formic acid). El-mass spectra were recorded on a Finnigan MS 8500 mass spectrometer. ${ }^{1} \mathrm{H}$ and ${ }^{13} \mathrm{C}$ NMR spectra were recorded on Jeol JNM-EX-270, Bruker Avance-360 and on Bruker Avance DRX-500 spectrometers. [D6]-DMSO $\left(\delta\left({ }^{1} \mathrm{H}\right)=2.49 \mathrm{ppm}, \delta\left({ }^{13} \mathrm{C}\right)=39.5 \mathrm{ppm}\right)$, $\mathrm{CD}_{3} \mathrm{OD}\left(\delta\left({ }^{1} \mathrm{H}\right)=3.35 \mathrm{ppm}, \delta\left({ }^{13} \mathrm{C}\right)=49.3 \mathrm{ppm}\right)$ and $\mathrm{D}_{2} \mathrm{O}$ were used as solvents, the internal standard was [D $\mathrm{D}_{6}$ DMSO. Chemical shifts are given in ppm relative to $\mathrm{SiMe}_{4}\left(\delta\left({ }^{1} \mathrm{H}\right)=0 \mathrm{ppm}\right)$, coupling constants are given in Hertz. ${ }^{1} \mathrm{H}$, Jmod, HH-COSY, HMQC-COSY, NOESY and HMBC experiments were used for assignment. Proton and carbon atoms of the indigo glycosides are assigned as shown below:

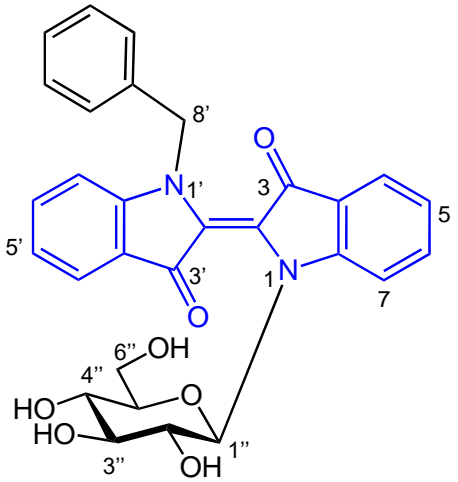

4

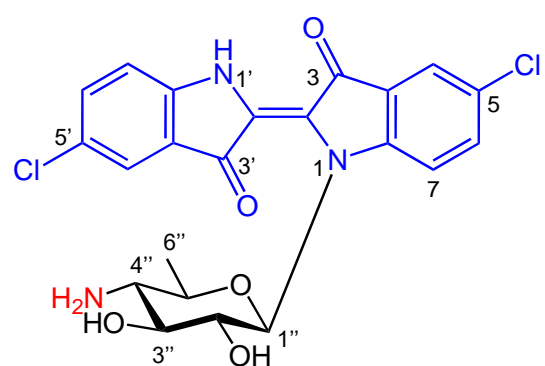

akashin $A(1)$

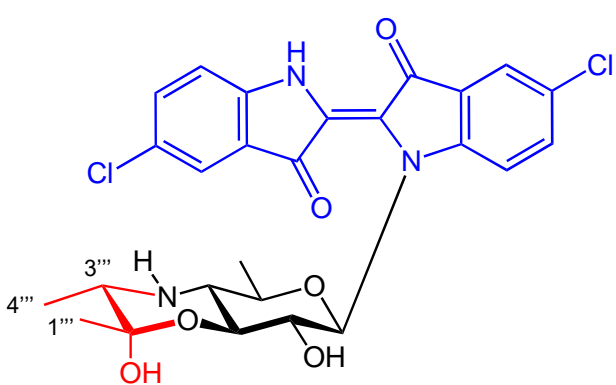

akashin C (3)

Scheme 12. NMR assignment of indigo glycosides.

3,4,6-Tri-O-acetyl-2-O-benzoyl- $\alpha$-D-glucopyranosyl-trichloroacetimidate (5). $20 \mathrm{~g}$ of 1,3,4,6-tetraacetylglucose 7 (57.5 mmol, $1.0 \mathrm{eq})$ were dissolved in dichloromethane $(200 \mathrm{~mL})$ and pyridine $(24 \mathrm{~mL})$. The solution was cooled to $0^{\circ} \mathrm{C}$ followed by dropwise addition of of benzoylchloride $(17 \mathrm{~mL}, 146 \mathrm{mmol})$ and subsequent stirring for $2.5 \mathrm{~h}$ at room temperature. The reaction was stopped by adding saturated potassium hydrogen carbonate $(100 \mathrm{~mL})$ and crushed ice $(350 \mathrm{~g})$. The mixture was stirred for further $1.5 \mathrm{~h}$. The aqueous phase was extracted with dichloromethane $(2 \times 200 \mathrm{~mL})$ and the combined organic layers were extracted with $2 \mathrm{~N}$ hydrochloric acid $(2 \times 200 \mathrm{~mL})$ and saturated potassium hydrogen carbonate $(200 \mathrm{~mL})$. The organic phase was dried over $\mathrm{MgSO}_{4}$, concentrated in vacuo and purified by flash chromatography $(7 \times 18 \mathrm{~cm}$, eluent: cyclohexane/ethyl acetate $9: 1 \rightarrow 5: 1 \rightarrow 2: 1 \rightarrow 1: 1$ ). Yield of 1,3,4,6-tetra-O-acetyl-2-O-benzoyl- $\alpha-D-$ glucopyranoside 7a: $25.9 \mathrm{~g}(99 \%, \alpha: \beta=9: 1) . R_{f}=0.49$ (cyclohexane/ethyl acetate $\left.2: 1\right) \cdot[\alpha]_{D} 22=+113.5^{\circ}(c=$ 1.02, dichloromethane). ESI/MS: $m / z 475.24[\mathrm{M}+\mathrm{Na}]^{+}$, (calcd for $\mathrm{C}_{19} \mathrm{H}_{24} \mathrm{O}_{11} \mathrm{Na}$, 475.12). ${ }^{1} \mathrm{H}-\mathrm{NMR}\left(270 \mathrm{MHz},\left[\mathrm{D}_{6}\right]-\right.$ DMSO) $\alpha$-anomer: $\delta=7.86\left(\mathrm{~d}, 2 \mathrm{H}, J=7.6 \mathrm{~Hz}, \mathrm{H}_{o}-\mathrm{Ar}\right.$ ), 7.69 (dd, $1 \mathrm{H}, J=7.6 \mathrm{~Hz}, \mathrm{H}_{\mathrm{p}}$-Ar), $7.54(\mathrm{dd}, 2 \mathrm{H}, J=7.6 \mathrm{~Hz}$, $\mathrm{H}_{m}-\mathrm{Ar}$ ), $6.35\left(\mathrm{~d}, 1 \mathrm{H}, J_{1,2}=3.6 \mathrm{~Hz}, \mathrm{H}-1\right), 5.53\left(\mathrm{dd}, 1 \mathrm{H}, J_{2,3}=J_{3,4}=9.9 \mathrm{~Hz}, \mathrm{H}-3\right), 5.28\left(\mathrm{dd}, 1 \mathrm{H}, J_{1,2}=3.6 \mathrm{~Hz}, J_{2,3}=9.9\right.$ $\mathrm{Hz}, \mathrm{H}-2$ ), 5.20 (dd, $\left.1 \mathrm{H}, J_{3,4}=J_{4,5}=9.9 \mathrm{~Hz}, \mathrm{H}-4\right), 4.25-4.18(\mathrm{~m}, 2 \mathrm{H}, \mathrm{H}-5, \mathrm{H}-6 \mathrm{a}), 4.07-4.01$ (m, $\left.1 \mathrm{H}, \mathrm{H}-6 \mathrm{~b}\right), 2.17$ (s, 3H, Ac), $2.03(\mathrm{~s}, 6 \mathrm{H}, 2 \mathrm{Ac}), 1.93(\mathrm{~s}, 3 \mathrm{H}, \mathrm{Ac}) .{ }^{13} \mathrm{C}-\mathrm{NMR}$ (68 MHz, [D6]-DMSO): $\delta=170.6,170.3,169.7,169.5$ (CO-Ac), 165.2 (CO-Bz), 134.5 ( $\mathrm{C}_{p}-\mathrm{Ar}$ ), 129.8 ( $\mathrm{C}_{m}-\mathrm{Ar}$ ), 129.5 ( $\mathrm{C}_{o}-\mathrm{Ar}$ ), 128.9 ( $\left.\mathrm{C}_{i}-\mathrm{Ar}\right), 88.9$ (C-1), 70.3 (C-5), 69.9 (C-2), 69.8 (C-3), 67.7 (C-4), 61.9 (C-6), 21.1, 20.9, 20.8 (AC).

To a stirred solution of tetraacetyl-2-benzoyl- $\alpha$-D-glucose $7 \mathrm{a}(8.70 \mathrm{~g}, 19.4 \mathrm{mmol}, 1.0 \mathrm{eq})$ in $\mathrm{N}, \mathrm{N}$ dimethylformamide $(25 \mathrm{~mL})$ was added hydrazine acetate $(2.67 \mathrm{~g}, 29 \mathrm{mmol}, 1.5 \mathrm{eq})$ of over 15 minutes. After 90 minutes the reaction was stopped by addition of acetone $(12 \mathrm{~mL})$ and stirred until the excess of hydrazine acetate was dissolved. The solution was diluted with dichloromethane $(400 \mathrm{~mL})$ and extracted with water and 
saturated potassium hydrogen carbonate. The organic layer was dried over $\mathrm{MgSO}_{4}$, concentrated in vacuo and purified by flash chromatography $(5 \times 18 \mathrm{~cm}$, eluent: cyclohexane/acetone $3: 1 \rightarrow 2: 1)$. Yield of 3,4,6-tri-Oacetyl-2-O-benzoyl-D-glucopyranose 7b: $6.22 \mathrm{~g}$ (80\%). $R_{f}=0.40$ (cyclohexane/acetone 2:1). $[\alpha]_{\mathrm{D}}{ }^{26}=+115.3^{\circ}(\mathrm{c}$ $=0.52$, dichloromethane). ESI/MS: $m / z 433.20[\mathrm{M}+\mathrm{Na}]^{+}$(calcd for $\left.\mathrm{C}_{19} \mathrm{H}_{22} \mathrm{O}_{10} \mathrm{Na}, 433.11\right) .{ }^{1} \mathrm{H} \mathrm{NMR}(270 \mathrm{MHz}$, [D6]-DMSO) $\alpha$-anomer: $\delta=7.90\left(\mathrm{~d}, 2 \mathrm{H}, J=7.6 \mathrm{~Hz}, \mathrm{H}_{o}\right.$-Ar), 7.69 (dd, $1 \mathrm{H}, J=7.6 \mathrm{~Hz}, \mathrm{H}_{p}$-Ar), 7.54 (dd, $2 \mathrm{H}, J=7.6$ $\mathrm{Hz}, \mathrm{H}_{m}-\mathrm{Ar}$ ), $7.38\left(\mathrm{~d}, 1 \mathrm{H}, J_{\mathrm{OH}, 1}=4.9 \mathrm{~Hz}, \mathrm{OH}\right), 5.57\left(\mathrm{dd}, 1 \mathrm{H}, J_{2,3}=J_{3,4}=9.9 \mathrm{~Hz}, \mathrm{H}-3\right), 5.43-5.39(\mathrm{~m}, 1 \mathrm{H}, \mathrm{H}-1), 5.02$ (dd, $\left.1 \mathrm{H}, J_{3,4}=J_{4,5}=9.9 \mathrm{~Hz}, \mathrm{H}-4\right), 4.93\left(\mathrm{dd}, 1 \mathrm{H}, J_{1,2}=4.9 \mathrm{~Hz}, J_{2,3}=9.9 \mathrm{~Hz}, \mathrm{H}-2\right), 4.25-4.11(\mathrm{~m}, 2 \mathrm{H}, \mathrm{H}-5, \mathrm{H}-6 \mathrm{a}), 4.10-$ 4.00 (m, 1H, H-6b), 2.02 (s, 6H, 2Ac), 1.91 (s, 3H, Ac). ${ }^{13} \mathrm{C} \mathrm{NMR} \mathrm{(68} \mathrm{MHz,} \mathrm{[D6]-DMSO):} \delta=170.7,170.3,169.9$ (CO-Ac), 165.5 (CO-Bz), 134.4 ( $\left.\mathrm{C}_{p}-\mathrm{Ar}\right), 129.8$ ( $\mathrm{C}_{m}-\mathrm{Ar}$ ), 129.4 ( $\left.\mathrm{C}_{o}-\mathrm{Ar}\right), 129.4\left(\mathrm{C}_{i}-\mathrm{Ar}\right), 88.6$ (C-1), 72.5 (C-2), 69.9 (C3), 68.8 (C-4), 67.0 (C-5), 62.7 (C-6), 21.1, 21.0, 20.9 (Ac).

Hemiacetal 7b (11.65 g, $28.4 \mathrm{mmol}, 1.0 \mathrm{eq})$ was dissolved in absolute dichloromethane $(160 \mathrm{~mL})$ and cooled to $0^{\circ} \mathrm{C}$. Trichloroacetonitrile $(28.5 \mathrm{~mL}, 283.9 \mathrm{mmol}, 10 \mathrm{eq})$ was added followed by DBU $(0.85 \mathrm{~mL}, 5.68 \mathrm{mmol}$, $0.2 \mathrm{eq})$. After 60 minutes the solvent was concentrated in vacuo $\left(20^{\circ} \mathrm{C}\right)$ and the residue was purified by flash chromatography $(7.5 \times 11 \mathrm{~cm}$, eluent: cyclohexane/acetone 5:1). Yield of imidate 5: $14.20 \mathrm{~g}(90.3 \%)$.

$\mathrm{R}_{\mathrm{f}}=0.52$ (cyclohexane/ethyl acetate 2:1). $[\alpha]_{\mathrm{D}}^{28}=-5.5^{\circ}$ (c = 0.53, dichloromethane). ESI/MS: $\mathrm{m} / \mathrm{z} 576.14$ $\left[\left({ }^{35} \mathrm{Cl}\right) \mathrm{M}+\mathrm{Na}\right]^{+}, \quad 578.14 \quad\left[\left({ }^{37} \mathrm{Cl}\right) \mathrm{M}+\mathrm{Na}\right]^{+}$(calcd for $\left.\mathrm{C}_{21} \mathrm{H}_{22} \mathrm{Cl}_{3} \mathrm{NO}_{10} \mathrm{Na}, \quad 576.02\right) .{ }^{1} \mathrm{H}-\mathrm{NMR} \quad\left(270 \mathrm{MHz},\left[\mathrm{D}_{6}\right]-\right.$ DMSO): $\delta=9.96$ (s, $1 \mathrm{H}, \mathrm{NH}$ ), 7.88 (d, 2H, $J=7.4 \mathrm{~Hz}, \mathrm{H}_{o}-\mathrm{Ar}$ ), 7.67 (dd, $1 \mathrm{H}, J=7.4 \mathrm{~Hz}, \mathrm{H}_{p}-\mathrm{Ar}$ ), 7.52 (dd, $2 \mathrm{H}, J=7.4$ $\mathrm{Hz}, \mathrm{H}_{m}-\mathrm{Ar}$ ), 6.62 (bs, $\left.1 \mathrm{H}, \mathrm{H}-1\right), 5.62$ (dd, $1 \mathrm{H}, J_{2,3}=J_{3,4}=9.9 \mathrm{~Hz}, \mathrm{H}-3$ ), 5.40 (dd, $1 \mathrm{H}, J_{1,2}=2.3 \mathrm{~Hz}, J_{2,3}=9.9 \mathrm{~Hz}, \mathrm{H}-2$ ), $5.26\left(\mathrm{dd}, 1 \mathrm{H}, J_{3,4}=J_{4,5}=9.9 \mathrm{~Hz}, \mathrm{H}-4\right), 4.27-4.08(\mathrm{~m}, 3 \mathrm{H}, \mathrm{H}-5, \mathrm{H}-6 \mathrm{a}, \mathrm{H}-6 \mathrm{~b}), 2.03(\mathrm{~s}, 6 \mathrm{H}, 2 \mathrm{xAc}), 1.93(\mathrm{~s}, 3 \mathrm{H}, \mathrm{Ac}) .{ }^{13} \mathrm{C}-$ NMR (68 MHz, [D 6 -DMSO): $\delta=170.5,170.2,169.8$ (CO-Ac), 165.1 (CO-Bz), 134.6 (C $\left.c_{p}-A r\right), 129.8$ (C $\left.\mathrm{C}_{m}-\mathrm{Ar}\right), 129.5$ ( $\mathrm{C}_{0}-\mathrm{Ar}$ ), 128.8 (Ci-Ar), 92.5 (C-1), 70.5 (C-2, C-5), 69.7 (C-3), 67.7 (C-4), 61.9 (C-6), 21.1, 20.8 (AC).

$\mathbf{N}$-Benzylindigo (6). Indigo $8(1 \mathrm{~g}, 3.81 \mathrm{mmol}, 1.0 \mathrm{eq})$ was suspended in absolute $\mathrm{N}, \mathrm{N}$-dimethylformamide $(25 \mathrm{~mL}), \mathrm{NaH}$ (60\% in oil suspension, $100 \mathrm{mg}, 2.5 \mathrm{mmol}, 0.66$ eq.) was added and the suspension stirred at room temperature. After 30 minutes further $\mathrm{NaH}$ (60\% in oil suspension, $100 \mathrm{mg}, 2.5 \mathrm{mmol}, 0.66$ eq.) was added. After $2 \mathrm{~h}$ benzylbromide $(540 \mu \mathrm{L}, 4.55 \mathrm{mmol}, 1.2 \mathrm{eq})$ was added dropwise, and the reaction was stirred for $1 \mathrm{~h}$ at room temperature. Subsequently, the reaction mixture was concentrated in vacuo $\left(60^{\circ} \mathrm{C}\right)$, the residue was dissolved in dichloromethane $(200 \mathrm{~mL})$ and extracted with water $(3 \mathrm{x})$. The organic layer was dried over $\mathrm{MgSO}_{4}$, concentrated in vacuo and purified by flash chromatography $(3.5 \mathrm{~cm} \times 28 \mathrm{~cm}$, eluent: toluene). Yield of 6: $641 \mathrm{mg}(48 \%)$, blue solid. $R_{f}=0.57$ (toluene/ethyl acetate 15:1). UV/VIS: $\lambda_{\max }(\lg \varepsilon)=626 \mathrm{~nm}(3.89)$ in dichloromethane. El/MS $(70 \mathrm{eV}): \mathrm{m} / z(\%)=352(54)[\mathrm{M}]^{+}$, (calcd for $\left.\mathrm{C}_{23} \mathrm{H}_{16} \mathrm{~N}_{2} \mathrm{O}_{2}, 352.12\right) .{ }^{1} \mathrm{H}-\mathrm{NMR}(270 \mathrm{MHz}$,

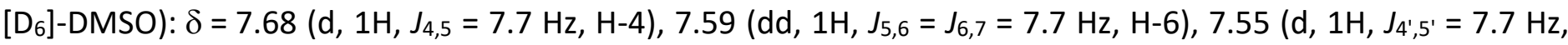
$\left.\mathrm{H}-4^{\prime}\right), 7.50\left(\mathrm{dd}, 1 \mathrm{H}, J_{5^{\prime}, 6^{\prime}}=J_{6^{\prime}, 7^{\prime}}=7.7 \mathrm{~Hz}, \mathrm{H}-6^{\prime}\right), 7.37\left(\mathrm{~d}, 1 \mathrm{H}, J_{6,7}=7.7 \mathrm{~Hz}, \mathrm{H}-7\right), 7.35\left(\mathrm{~d}, 1 \mathrm{H}, J_{6^{\prime}, 7^{\prime}}=7.7 \mathrm{~Hz}, \mathrm{H}-7^{\prime}\right)$, 7.26-7.16 (m, 3H, $\mathrm{H}_{m}-\mathrm{Ar}, \mathrm{H}_{p^{-}}-\mathrm{Ar}$ ), 7.12-7.07 (m, 2H, $\mathrm{H}_{o}-\mathrm{Ar}$ ), 7.06 (dd, $\left.1 \mathrm{H}, J_{4,5}=7.7 \mathrm{~Hz}, \mathrm{H}-5\right), 6.94$ (dd, $1 \mathrm{H}, J_{4^{\prime}, 5^{\prime}}=$ $\left.7.7 \mathrm{~Hz}, \mathrm{H}-5^{\prime}\right), 5.78\left(\mathrm{~s}, 2 \mathrm{H}, \mathrm{H}-8^{\prime}\right) .{ }^{13} \mathrm{C}-\mathrm{NMR}\left(68 \mathrm{MHz},\left[\mathrm{D}_{6}\right]-\mathrm{DMSO}\right): \delta=188.8\left(\mathrm{C}_{\mathrm{q}^{-}} 3^{\prime}\right), 187.2\left(\mathrm{C}_{\mathrm{q}^{-}}-3\right), 153.3,152.5\left(\mathrm{C}_{\mathrm{q}^{-}}\right.$ 7a, $\left.C_{q^{-}}-7 a^{\prime}\right), 138.3$ ( $\left.C_{i}-A r\right), 136.7,136.5$ (C-6, C-6'), 129.1, 127.7, 127.3 (C-Ar), $125.0\left(C_{q^{-}} 2^{\prime}\right), 124.4,124.0$ (C-4, C$\left.4^{\prime}\right), 123.3\left(C_{q}-2\right), 121.7,121.0$ (C-5, $\left.C-5 '\right), 121.2$ ( $\left.C_{q}-3 a^{\prime}\right), 119.6$ ( $\left.C_{q}-3 a\right), 113.8\left(C-7^{\prime}\right), 112.7(C-7), 50.3\left(C_{2}-8{ }^{\prime}\right)$.

$\mathbf{N}$-[(2,3,4-Tri-O-acetyl-2-O-benzoyl- $\beta$-D-glucopyranosyl)- $\mathbf{N}$ '-benzyl]-indigo (9). Under an inert gas imidate 5 (764 mg, $1.38 \mathrm{mmol}, 1.0 \mathrm{eq}$ ), $N$-benzylindigo 6 (500 mg, $1.42 \mathrm{mmol}, 1.03 \mathrm{eq}$ ) and molecular sieves $4 \AA$ (1.0 g) were suspended in absolute dichloromethane $(10 \mathrm{~mL})$, stirred for 30 minutes at room temperature and cooled to $-18{ }^{\circ} \mathrm{C}$. The reaction was started by adding TMSOTf $(100 \mu \mathrm{L}, 0.55 \mathrm{mmol}, 0.4 \mathrm{eq})$. After $3 \mathrm{~h}$ at $-18{ }^{\circ} \mathrm{C}$ the suspension was slowly warmed to room temperature over $12 \mathrm{~h}$. The reaction was quenched by adding saturated potassium hydrogen carbonate $(10 \mathrm{~mL})$. After 10 minutes the mixture was filtered over Celite and washed with dichloromethane. The organic filtrate was extracted with water, saturated potassium hydrogen carbonate and brine. The organic layer was dried $\left(\mathrm{MgSO}_{4}\right)$ and filtered. The filtrate was concentrated in vacuo 
and the residue purified by flash chromatography $(5 \times 20 \mathrm{~cm}$, solvent: toluene/ethyl acetate 15:1 $\rightarrow 12: 1 \rightarrow$ 9:1). Unreacted $\mathrm{N}$-benzylindigo 6 was recovered $124 \mathrm{mg}(0.35 \mathrm{mmol}, 25 \%)$. Yield of 9: $232 \mathrm{mg}(22.6 \%)$, blue solid. $R_{f}=0.27$ (cyclohexane/ethyl acetate 1.5:1). UV/VIS: $\lambda$ max $(\lg \varepsilon)=630 \mathrm{~nm}$ (3.80) in dichloromethane. $[\alpha]_{D^{23}}=+5428.6^{\circ}$ ( $c=7.0 \mu \mathrm{g} / \mathrm{mL}$, dichloromethane). ESI/MS: $\mathrm{m} / z 745.76[\mathrm{M}+\mathrm{H}]^{+}$(calcd for $\mathrm{C}_{42} \mathrm{H}_{37} \mathrm{~N}_{2} \mathrm{O}_{11}$, 745.24). ${ }^{1} \mathrm{H}-\mathrm{NMR}\left(360 \mathrm{MHz},\left[\mathrm{D}_{6}\right]-\mathrm{DMSO}\right): \delta=7.73$ (d, $\left.1 \mathrm{H}, J_{6,7}=7.9 \mathrm{~Hz}, \mathrm{H}-7\right), 7.69\left(\mathrm{~d}, 1 \mathrm{H}, J_{4^{\prime}, 5^{\prime}}=7.6 \mathrm{~Hz}, \mathrm{H}-4^{\prime}\right), 7.69$ (dd, $2 \mathrm{H}, J=7.9 \mathrm{~Hz}, \mathrm{H}-6, \mathrm{H}_{p}-\mathrm{Ar}$ ), 7.54 (dd, $2 \mathrm{H}, J=8.0 \mathrm{~Hz}, \mathrm{H}_{m}-\mathrm{Ar}$ ), 7.47 (d, 1H, J4,5 = 7.3 Hz, H-4), 7.40 (dd, $2 \mathrm{H}, J=$ $8.0 \mathrm{~Hz}, \mathrm{H}-6^{\prime}, \mathrm{H}_{p^{-}}-\mathrm{Ar}$ ), 7.35-7.32 (m, $\left.4 \mathrm{H}, \mathrm{H}_{o^{-}}-\mathrm{Ar}\right), 7.11$ (dd, $\left.1 \mathrm{H}, J_{4,5}=7.3 \mathrm{~Hz}, J_{5,6}=7.9 \mathrm{~Hz}, \mathrm{H}-5\right), 7.03\left(\mathrm{dd}, 1 \mathrm{H}, J_{4^{\prime}, 5^{\prime}}=\right.$ $\left.7.6 \mathrm{~Hz}, J_{5^{\prime}, 6^{\prime}}=8.0 \mathrm{~Hz}, \mathrm{H}-5^{\prime}\right), 6.92\left(\mathrm{dd}, 2 \mathrm{H}, J=7.7 \mathrm{~Hz}, \mathrm{H}_{m}-\mathrm{Ar}\right), 6.66\left(\mathrm{~d}, 1 \mathrm{H}, J_{6^{\prime}, 7^{\prime}}=8.0 \mathrm{~Hz}, \mathrm{H}-7^{\prime}\right), 6.01\left(\mathrm{~d}, 1 \mathrm{H}, J_{1^{\prime \prime}, 2^{\prime \prime}}=\right.$ $\left.8.7 \mathrm{~Hz}, \mathrm{H}-1^{\prime \prime}\right), 5.74-5.68\left(\mathrm{~m}, 2 \mathrm{H}, \mathrm{H}-2^{\prime \prime}, \mathrm{H}-3^{\prime \prime}\right), 5.33\left(\mathrm{dd}, 1 \mathrm{H}, J_{3^{\prime \prime}} 4^{\prime \prime}=J_{4^{\prime \prime}, 5^{\prime \prime}}=9.4 \mathrm{~Hz}, \mathrm{H}-4^{\prime \prime}\right), 5.02\left(\mathrm{~d}, 1 \mathrm{H}, J_{\text {gem }}=16.8\right.$ $\left.\mathrm{Hz}, \mathrm{H}-8^{\prime}\right), 4.71\left(\mathrm{~d}, 1 \mathrm{H}, J_{\text {gem }}=16.8 \mathrm{~Hz}, \mathrm{H}-8^{\prime}\right)$, 4.31-4.26 (m, 1H, H-5"), 4.23-4.14 (m, 1H, H-6a"), 4.08-4.02 (m, $1 \mathrm{H}$, H-6b"), $2.22(\mathrm{~s}, 3 \mathrm{H}, \mathrm{Ac}), 2.03(\mathrm{~s}, 3 \mathrm{H}, \mathrm{Ac}), 1.74(\mathrm{~s}, 3 \mathrm{H}, \mathrm{Ac}) .{ }^{13} \mathrm{C}-\mathrm{NMR}\left(90 \mathrm{MHz},\left[\mathrm{D}_{6}\right]-\mathrm{DMSO}\right): \delta=185.1\left(\mathrm{C}_{\mathrm{q}^{-}} 3^{\prime}\right)$,

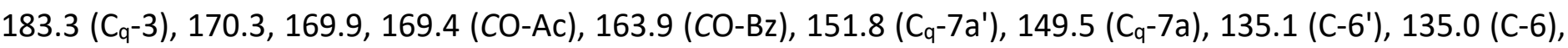

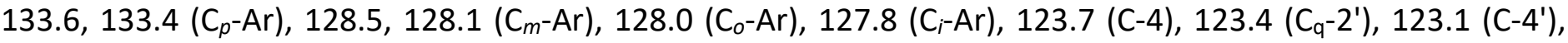
122.2 (C-5), 121.7 ( $\mathrm{C}_{\mathrm{q}}-2$ ), 121.4 (C-5'), 119.0 (C-3a, C-3a'), 115.7 (C-7), 112.7 (C-7'), 87.5 (C-1"), 74.1 (C-5"), 73.1 (C-3"), 68.1 (C-2"), 68.6 (C-4"), 61.4 (C-6"), 53.5 ( $\mathrm{CH}_{2}-8$ '), 20.6, 20.4, 20.2 (Ac).

$\boldsymbol{N}$-(2,3,4-Tri-O-acetyl-2-O-benzoyl- $\boldsymbol{\beta}$-D-glucopyranosyl)-indigo (9b). Glycoside 9 (320 mg, $0.43 \mathrm{mmol}, 1.0$ eq) was dissolved in toluene/acetic acid $(8 \mathrm{~mL}, 1: 1)$ and stirred in an open flask for $28 \mathrm{~h}$ at $50^{\circ} \mathrm{C}$. The solution was diluted with dichloromethane $(150 \mathrm{~mL})$ and extracted with water and saturated potassium hydrogen carbonate. The organic layer was dried over $\mathrm{MgSO}_{4}$, concentrated in vacuo and purified by flash chromatography ( $5 \times 18 \mathrm{~cm}$, eluent: toluene/ethyl acetate $15: 1 \rightarrow 12: 1)$. Yield: $173 \mathrm{mg}(61.4 \%)$, blue solid. $\mathrm{R}_{\mathrm{f}}=$ 0.41 (toluene/ethyl acetate 5:1). UV/VIS: $\lambda_{\max }(\lg \varepsilon)=600 \mathrm{~nm}(4.04)$ in dichloromethane. $[\alpha]_{D}^{23}=+2328.2^{\circ}(c=$ $3.9 \mu \mathrm{g} / \mathrm{mL}$, dichloromethane). ESI/MS: $\mathrm{m} / z 655.30[\mathrm{M}+\mathrm{H}]^{+}$(calcd for $\left.\mathrm{C}_{35} \mathrm{H}_{31} \mathrm{~N}_{2} \mathrm{O}_{11}, 655.19\right) .{ }^{1} \mathrm{H}-\mathrm{NMR}(360 \mathrm{MHz}$, [D6]-DMSO): $\delta=10.75$ (s, $1 \mathrm{H}, \mathrm{N}^{\prime} H$ ), $7.76\left(\mathrm{~d}, 1 \mathrm{H}, J_{6,7}=7.5 \mathrm{~Hz}, \mathrm{H}-7\right.$ ), 7.68 (dd, $\left.1 \mathrm{H}, J_{5,6}=J_{6,7}=7.5 \mathrm{~Hz}, \mathrm{H}-6\right), 7.67$ (d, $\left.1 \mathrm{H}, J_{4^{\prime}, 5^{\prime}}=7.5 \mathrm{~Hz}, \mathrm{H}-4^{\prime}\right), 7.57-7.50\left(\mathrm{~m}, 5 \mathrm{H}, \mathrm{H}-4, \mathrm{H}-6^{\prime}, \mathrm{H}_{o^{-}}-\mathrm{Ar}, \mathrm{H}_{p^{-}}-\mathrm{Ar}\right), 7.34\left(\mathrm{~d}, 1 \mathrm{H}, J_{6^{\prime}, 7^{\prime}}=7.6 \mathrm{~Hz}, \mathrm{H}-7^{\prime}\right), 7.30$ (dd, $1 \mathrm{H}, J$ $=7.6 \mathrm{~Hz}, \mathrm{H}_{m}-\mathrm{Ar}$ ), $7.14\left(\mathrm{dd}, 1 \mathrm{H}, J_{4,5}=J_{5,6}=7.5 \mathrm{~Hz}, \mathrm{H}-5\right), 7.00\left(\mathrm{dd}, 1 \mathrm{H}, J_{4^{\prime}, 5^{\prime}}=J_{5^{\prime}, 6^{\prime}}=7.6 \mathrm{~Hz}, \mathrm{H}-5^{\prime}\right), 6.56\left(\mathrm{~d}, 1 \mathrm{H}, J_{1^{\prime \prime}, 2^{\prime \prime}}=\right.$ $\left.9.1 \mathrm{~Hz}, \mathrm{H}-1^{\prime \prime}\right), 5.60$ (dd, $\left.1 \mathrm{H}, J_{1^{\prime \prime}, 2^{\prime \prime}}=J_{2^{\prime \prime}, 3^{\prime \prime}}=9.1 \mathrm{~Hz}, \mathrm{H}-2^{\prime \prime}\right), 5.52\left(\mathrm{dd}, 1 \mathrm{H}, J_{2^{\prime \prime}, 3^{\prime \prime}}=J_{3^{\prime \prime}} 4^{\prime \prime}=9.1 \mathrm{~Hz}, \mathrm{H}-3^{\prime \prime}\right), 5.36$ (dd, $1 \mathrm{H}$, $\left.J_{3^{\prime \prime}, 4^{\prime \prime}}=J_{4^{\prime \prime}, 5^{\prime \prime}}=9.1 \mathrm{~Hz}, \mathrm{H}-4^{\prime \prime}\right), 4.34-4.28\left(\mathrm{~m}, 2 \mathrm{H}, \mathrm{H}-6 \mathrm{a}^{\prime \prime}, \mathrm{H}-6 \mathrm{~b}^{\prime \prime}\right), 4.23-4.17\left(\mathrm{~m}, 1 \mathrm{H}, \mathrm{H}-5^{\prime \prime}\right), 2.13$ (s, 3H, Ac), $2.04(\mathrm{~s}$, $3 \mathrm{H}, \mathrm{Ac}), 1.78(\mathrm{~s}, 3 \mathrm{H}, \mathrm{Ac}) .{ }^{13} \mathrm{C}-\mathrm{NMR}\left(90 \mathrm{MHz},\left[\mathrm{D}_{6}\right]-\mathrm{DMSO}\right): \delta=186.9\left(\mathrm{C}_{\mathrm{q}}-3^{\prime}\right), 186.8\left(\mathrm{C}_{\mathrm{q}}-3\right), 170.1,169.4$ (CO-Ac), 163.9 (CO-Bz), 152.4 ( $\left.C_{q^{-}}-7 a^{\prime}\right), 149.8$ ( $\left.C_{q}-7 a\right), 136.5$ (C-6'), $135.7(C-6), 133.6\left(C_{p}-A r\right), 129.0\left(C_{m}-A r\right), 128.6\left(C_{o}-A r\right)$, 127.9 ( $\left.C_{i}-A r\right), 124.6$ ( $\left.C_{q}-2^{\prime}\right), 124.2$ (C-4'), 123.3 (C-4), 122.6 (C-5), $122.1\left(C_{q}-2\right), 120.8$ (C-5'), 119.0 (C-3a, C-3a'), 116.2 (C-7), 113.3 (C-7'), 85.1 (C-1"), 74.2 (C-5"), 73.0 (C-3"), 68.7 (C-2"), 68.0 (C-4"), 61.9 (C-6"), 20.6, 20.4, 20.2 (AC).

$\boldsymbol{N}$ - $\boldsymbol{\beta}$-D-Glucopyranosyl)-indigo (4). Under an argon atmosphere indigo $9 \mathrm{~b}$ (179 mg, $0.27 \mathrm{mmol}, 1.0$ eq) was dissolved in absolute methanol $(9 \mathrm{~mL})$. NaOMe in $\mathrm{MeOH}(9 \mathrm{~mL}, \mathrm{c}=0.01 \mathrm{~mol} / \mathrm{L})$ was added and the reaction was stirred for $11 \mathrm{~h}$ at room temperature. The reaction was stopped by neutralization with Amberlyst $15\left(\mathrm{H}^{+}\right)$. The ion exchange resin was removed by filtration over Celite and washed with methanol. The filtrate was concentrated and purified in three aliquots by solid phase extraction (SepPak- $\mathrm{C}_{18}, 2 \mathrm{~g}$ ) with a gradient of 5-15\% acetonitrile in water in 1\%-steps. The product eluted between $12-15 \%$ acetonitrile and was lyophilized. Yield: $58 \mathrm{mg}$ (50.7\%), blue solid. $\mathrm{R}_{\mathrm{f}}$ : 0.25 (dichloromethane/methanol 10:1). UV/VIS: $\lambda_{\max }(\lg \varepsilon)=606 \mathrm{~nm}(4.08)$ in methanol. $[\alpha]_{D}{ }^{27}=+2258^{\circ}\left(c=6.2 \mu \mathrm{g} / \mathrm{mL}\right.$, methanol). ESI/MS: $\mathrm{m} / z 425.07[\mathrm{M}+\mathrm{H}]^{+}$(calcd for $\mathrm{C}_{22} \mathrm{H}_{21} \mathrm{~N}_{2} \mathrm{O}_{7}$, 425.13). ${ }^{1} \mathrm{H}-\mathrm{NMR}\left(500 \mathrm{MHz},\left[\mathrm{D}_{6}\right]-\mathrm{DMSO}\right.$ with $\left.\mathrm{D}_{2} \mathrm{O}\right): \delta=7.68\left(\mathrm{~d}, 1 \mathrm{H}, J_{4,5}=7.5 \mathrm{~Hz}, \mathrm{H}-4\right), 7.59\left(\mathrm{~d}, 1 \mathrm{H}, J_{6,7}=7.5 \mathrm{~Hz}\right.$, $\mathrm{H}-7$ ), $7.56\left(\mathrm{~d}, 1 \mathrm{H}, J_{4^{\prime}, 5^{\prime}}=7.7 \mathrm{~Hz}, \mathrm{H}-4^{\prime}\right), 7.55\left(\mathrm{dd}, 1 \mathrm{H}, J_{5,6}=J_{6,7}=7.5 \mathrm{~Hz}, \mathrm{H}-6\right), 7.51\left(\mathrm{~d}, 1 \mathrm{H}, J_{5^{\prime}, 6^{\prime}}=J_{6^{\prime}, 7^{\prime}}=7.7 \mathrm{~Hz}, \mathrm{H}-6^{\prime}\right)$, $7.33\left(\mathrm{~d}, 1 \mathrm{H}, J_{6^{\prime}, 7^{\prime}}=7.7 \mathrm{~Hz}, \mathrm{H}-7^{\prime}\right), 7.12\left(\mathrm{dd}, 1 \mathrm{H}, J_{4,5}=J_{5,6}=7.5 \mathrm{~Hz}, \mathrm{H}-5\right), 6.95\left(\mathrm{dd}, 1 \mathrm{H}, J_{4^{\prime}, 5^{\prime}}=J_{5^{\prime}, 6^{\prime}}=7.7 \mathrm{~Hz}, \mathrm{H}-5^{\prime}\right)$, $5.87\left(\mathrm{~d}, 1 \mathrm{H}, J_{1 ", 2 "}=9.1 \mathrm{~Hz}, \mathrm{H}-1^{\prime \prime}\right), 3.80\left(\mathrm{dd}, 1 \mathrm{H}, J_{5^{\prime \prime}, 6 \mathrm{a}}<1.0 \mathrm{~Hz}, J_{6 a ", 6 b^{\prime \prime}}=11.3 \mathrm{~Hz}, \mathrm{H}-6 \mathrm{a}^{\prime \prime}\right), 3.66(\mathrm{dd}, 1 \mathrm{H}$, 
$\left.J_{5^{\prime \prime}, 6 b^{\prime \prime}}=4.9 \mathrm{~Hz}, J_{6 \mathrm{a}^{\prime \prime}, 6 \mathrm{~b}^{\prime \prime}}=11.3 \mathrm{~Hz}, \mathrm{H}-6 \mathrm{~b}^{\prime \prime}\right), 3.51$ (dd, $\left.1 \mathrm{H}, J_{1^{\prime \prime}, 2^{\prime \prime}}=J_{2^{\prime \prime}, 3^{\prime \prime}}=9.1 \mathrm{~Hz}, \mathrm{H}-2^{\prime \prime}\right), 3.47-3.39\left(\mathrm{~m}, 1 \mathrm{H}, \mathrm{H}-5^{\prime \prime}\right), 3.31$ $\left(\mathrm{dd}, 1 \mathrm{H}, J_{3^{\prime \prime}, 4^{\prime \prime}}=J_{4^{\prime \prime}, 5^{\prime \prime}}=9.1 \mathrm{~Hz}, \mathrm{H}-4^{\prime \prime}\right), 3.19$ (dd, $\left.1 \mathrm{H}, J_{2^{\prime \prime}, 3^{\prime \prime}}=J_{3^{\prime \prime}, 4^{\prime \prime}}=9.1 \mathrm{~Hz}, \mathrm{H}-3^{\prime \prime}\right) .{ }^{1} \mathrm{H}-\mathrm{NMR}\left(360 \mathrm{MHz},\left[\mathrm{D}_{6}\right]-\mathrm{DMSO} \delta\right.$

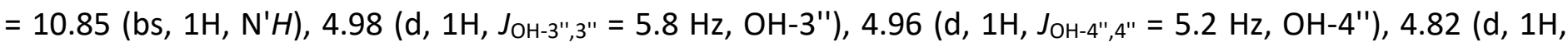
$\left.J_{\mathrm{OH}-2^{\prime \prime 2} 2^{\prime \prime}}=6.8 \mathrm{~Hz}, \mathrm{OH}-2^{\prime \prime}\right), 4.68\left(\mathrm{t}, 1 \mathrm{H}, \mathrm{J}_{\mathrm{OH}-6^{\prime \prime}, 6^{\prime \prime}}=5.7 \mathrm{~Hz}, \mathrm{OH}-6^{\prime \prime}\right) .{ }^{13} \mathrm{C}-\mathrm{NMR}\left(126 \mathrm{MHz},\left[\mathrm{D}_{6}\right]-\mathrm{DMSO}\right.$ with $\left.\mathrm{D}_{2} \mathrm{O}\right): \delta=188.2\left(\mathrm{C}_{\mathrm{q}}-3^{\prime}\right), 186.5\left(\mathrm{C}_{\mathrm{q}}-3\right), 152.1\left(\mathrm{C}_{\mathrm{q}^{-}}-7 \mathrm{a}^{\prime}\right), 150.8\left(\mathrm{C}_{\mathrm{q}^{-}}-7 \mathrm{a}\right), 136.0\left(\mathrm{C}-6^{\prime}\right), 135.1$ (C-6), 124.0 (C-4'), 123.9 $\left(C_{q}-2\right), 123.6\left(C_{q^{-}} 2^{\prime}\right), 123.2 \quad(C-4), 122.7\left(C_{q}-3 a\right), 121.7(C-5), 120.3(C-5), 119.1\left(C_{q}-3 a^{\prime}\right), 116.6(C-7), 113.1(C-$ 7'), 88.5 (C-1"), 80.7 (C-5"), 78.0 (C-3"), 70.2, 70.1 (C-2", C-4"), 61.4 (C-6").

1,2,3-Tri-O-benzoyl-4,6-O-benzylidene-D-galactopyranose (12). The synthesis of 12 was carried out following the procedure of Gros and Deulofeu. ${ }^{26}$ The anomers obtained after benzoylation of 4,6-benzylidene-Dgalactose $(10 \mathrm{~g})$ were separated by flash chromatography $(7.5 \times 12 \mathrm{~cm}$, eluent: cyclohexane/ethyl acetate 9:1 $\rightarrow 6: 1 \rightarrow 5: 1 \rightarrow 3: 1 \rightarrow 2: 1 \rightarrow 1: 1$ ). $\alpha$-anomer $12 \alpha$ : yield: $18.88 \mathrm{~g}$ (87\%). $\mathrm{R}_{\mathrm{f}}=0.62$ (dichloromethane/methanol 2:1). $[\alpha]_{D}{ }^{24}=-79.2^{\circ}$ ( $c=0.50$, dichloromethane). ESI/MS: $m / z 603.21[\mathrm{M}+\mathrm{Na}]^{+}$(calcd for $\left.\mathrm{C}_{34} \mathrm{H}_{28} \mathrm{O}{ }_{9} \mathrm{Na}, 603.16\right)$ ${ }^{1} \mathrm{H}-\mathrm{NMR}\left(270 \mathrm{MHz},\left[\mathrm{D}_{6}\right]-\mathrm{DMSO}\right): \delta=8.14(\mathrm{~d}, 2 \mathrm{H}, J=7.3 \mathrm{~Hz}, \mathrm{H}-\mathrm{Ar}$ ), 7.88 (d, 2H, J= $7.3 \mathrm{~Hz}, \mathrm{H}-\mathrm{Ar}$ ), 7.74 (d, $2 \mathrm{H}, J=$ $7.3 \mathrm{~Hz}, \mathrm{H}-\mathrm{Ar}$ ), 7.62 (dd, 3H, J = 7.7 Hz, H-Ar), 7.57-7.33 (m, 10H, H-Ar), 6.79 (d, $1 \mathrm{H}, J_{1,2}=3.4 \mathrm{~Hz}, \mathrm{H}-1$ ), 6.01 (dd, $\left.1 \mathrm{H}, J_{2,3}=10.9 \mathrm{~Hz}, J_{3,4}=3.1 \mathrm{~Hz}, \mathrm{H}-3\right), 5.80$ (dd, $\left.1 \mathrm{H}, J_{1,2}=3.4 \mathrm{~Hz}, J_{2,3}=10.9 \mathrm{~Hz}, \mathrm{H}-2\right), 5.78(\mathrm{~s}, 1 \mathrm{H}, \mathrm{H}-7), 4.87$ (dd, $\left.1 \mathrm{H}, J_{3,4}=J_{4,5}=3.1 \mathrm{~Hz}, \mathrm{H}-4\right), 4.41-4.39(\mathrm{~m}, 1 \mathrm{H}, \mathrm{H}-5), 4.22-4.14(\mathrm{~m}, 2 \mathrm{H}, \mathrm{H}-6 \mathrm{a}, \mathrm{H}-6 \mathrm{~b}) .{ }^{13} \mathrm{C}-\mathrm{NMR}\left(68 \mathrm{MHz},\left[\mathrm{D}_{6}\right]-\right.$

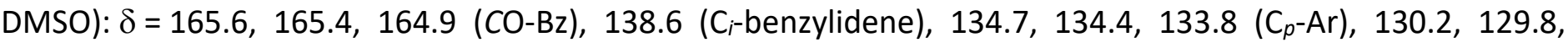
129.7, 129.6, 129.5 (C-Ar), 129.4 (C $\left.C_{-}-A r\right), 129.3$ (C-Ar), 129.2 (C $\left.C_{-}-A r\right), 129.1$ (C-Ar), 129.0 (C C $\left._{-}-A r\right), 128.8,126.6$ (CAr), 100.1 (C-7), 91.4 (C-1), 74.0 (C-4), 69.3 (C-3), 68.8 (C-6), 68.0 (C-2), 65.5 (C-5).

$\beta$-anomer 12 $\beta$ : yield: $2.72 \mathrm{~g}(13 \%) . \mathrm{R}_{\mathrm{f}}=0.49$ (dichloromethane/methanol $\left.2: 1\right) .[\alpha]_{\mathrm{D}}{ }^{24}=-14.2^{\circ}(\mathrm{c}=0.50$, dichloromethane). ${ }^{1} \mathrm{H}-\mathrm{NMR}\left(270 \mathrm{MHz},\left[\mathrm{D}_{6}\right]-\mathrm{DMSO}\right): \delta=8.00-7.80(\mathrm{~m}, 6 \mathrm{H}, \mathrm{H}-\mathrm{Ar}), 7.70-7.32(\mathrm{~m}, 14 \mathrm{H}, \mathrm{H}-\mathrm{Ar}), 6.45$ $\left(d, 1 \mathrm{H}, J_{1,2}=7.5 \mathrm{~Hz}, \mathrm{H}-1\right), 5.92\left(\mathrm{dd}, 1 \mathrm{H}, J_{3,2}=10.5 \mathrm{~Hz}, J_{3,4}=2.9 \mathrm{~Hz}, \mathrm{H}-3\right.$ ), 5.84 (dd, $1 \mathrm{H}, J_{1,2}=7.5 \mathrm{~Hz}, J_{2,3}=10.5 \mathrm{~Hz}$, $\mathrm{H}-2$ ), $5.76(\mathrm{~s}, 1 \mathrm{H}, \mathrm{H}-7), 4.79$ (dd, $\left.1 \mathrm{H}, J_{3,4}=J_{4,5}=2.9 \mathrm{~Hz}, \mathrm{H}-4\right), 4.27-4.18$ (m, 3H, H-5, H-6a, H-6b). ${ }^{13} \mathrm{C}-\mathrm{NMR}(68$ $\mathrm{MHz},\left[\mathrm{D}_{6}\right]$-DMSO): $\delta=165.6,165.5,164.7$ (CO-Bz), 138.6 ( $\mathrm{C}_{i}$-benzylidene), 134.8, 134.4, 133.4 ( $\mathrm{C}_{p}$-Ar), 129.9, 129.8, 129.7, 129.6, 129.4, (C-Ar), 129.3 (Ci-Ar), 129.2 (C-Ar), 129.1 (Ci-Ar), 128.7 (C-Ar), 128.6 (Ci-Ar), 126.5 (CAr), 100.1 (C-7), 92.9 (C-1), 73.6 (C-4), 72.1 (C-3), 69.3 (C-2), 68.5 (C-6), 67.6 (C-5).

1,2,3-Tri-O-benzoyl-6-deoxy-6-iodo- $\alpha$-D-galactopyranose (13 $\alpha$ ). Tribenzoyl-4,6-O-benzylidene- $\alpha-D$-galactose $12 \alpha(16.45 \mathrm{~g}, 28.4 \mathrm{mmol}, 1.0 \mathrm{eq})$ was dissolved in dry acetonitrile $(165 \mathrm{~mL})$ and absolute methanol $(107 \mathrm{~mL})$. $3.34 \mathrm{~g}(17.6 \mathrm{mmol}, 0.62 \mathrm{eq})$ of $p$-toluenesulfonic acid monohydrate in $34 \mathrm{~mL}$ of absolute methanol were added over 10 minutes. After $3 \mathrm{~h}$ of stirring $1.67 \mathrm{~g}(8.8 \mathrm{mmol}, 0.31 \mathrm{eq})$ of $p$-toluenesulfonic acid monohydrate in $17 \mathrm{~mL}$ of absolute methanol were added. After $21 \mathrm{~h}$ the reaction was quenched by adding $4.2 \mathrm{~mL}$ of triethylamine. The reaction mixture was concentrated in vacuo and the residue was purified by flash chromatography $(7.5 \times 16 \mathrm{~cm}$, eluent: cyclohexane/ethyl acetate $6: 1 \rightarrow 5: 1 \rightarrow 3: 1 \rightarrow 1: 1 \rightarrow 1: 2)$. Yield of 1,2,3tri-O-benzoyl- $\alpha$-D-galactopyranose $(\mathbf{1 2} \alpha-\mathbf{1}): 10.35 \mathrm{~g}$ (74.1\%). $\mathrm{R}_{\mathrm{f}}=0.24$ (cyclohexane/ethyl acetate 1.5:1). $[\alpha]_{D}{ }^{26}=+27^{\circ}$ (c = 0.50, dichloromethane). ESI/MS: $m / z 515.29[\mathrm{M}+\mathrm{Na}]^{+}$(calcd for $\left.\mathrm{C}_{27} \mathrm{H}_{24} \mathrm{O}_{9} \mathrm{Na}, 515.13\right) .{ }^{1} \mathrm{H}-$ NMR (270 MHz, [D 6 -DMSO): $\delta=8.09$ (d, 2H, J=7.9 Hz, H-Ar), 8.01 (d, 2H, J= 7.9 Hz, H-Ar), 7.77-7.69 (m, 3H, $\mathrm{H}-\mathrm{Ar}$ ), 7.66-7.43 (m, 6H, H-Ar), 7.37 (dd, $2 \mathrm{H}, J=7.7 \mathrm{~Hz}, \mathrm{H}-\mathrm{Ar}), 6.65\left(\mathrm{~d}, 1 \mathrm{H}, J_{1,2}=3.4 \mathrm{~Hz}, \mathrm{H}-1\right), 5.81\left(\mathrm{dd}, 1 \mathrm{H}, J_{1,2}=\right.$ $\left.3.4 \mathrm{~Hz}, J_{2,3}=10.5 \mathrm{~Hz}, \mathrm{H}-2\right), 5.70-5.73(\mathrm{~m}, 1 \mathrm{H}, \mathrm{OH}-4), 5.68\left(\mathrm{dd}, 1 \mathrm{H}, J_{2,3}=10.5 \mathrm{~Hz}, J_{3,4}=2.4 \mathrm{~Hz}, \mathrm{H}-3\right), 4.81(\mathrm{t}, 1 \mathrm{H}$, $\left.J_{\mathrm{OH}-6,6}=5.6 \mathrm{~Hz}, \mathrm{OH}-6\right), 4.44-4.33(\mathrm{~m}, 1 \mathrm{H}, \mathrm{H}-4), 4.21\left(\mathrm{dd}, 1 \mathrm{H}, J_{4,5}=J_{5,6}=6.0 \mathrm{~Hz}, \mathrm{H}-5\right), 3.69-3.52(\mathrm{~m}, 2 \mathrm{H}, \mathrm{H}-6 \mathrm{a}, \mathrm{H}-$ 6b). ${ }^{13} \mathrm{C}-\mathrm{NMR}$ (68 MHz, [D6]-DMSO): $\delta=165.8,165.6,164.9$ (CO-Bz), 134.7, 134.3, 134.1 (C $\left.\mathrm{C}_{p}-\mathrm{Ar}\right), 130.1$ (C-Ar), 130.0 ( $C_{i}$-Ar), 129.9, 129.6, 129.5 (C-Ar), 129.4 (C $\left.{ }_{i}-A r\right), 129.3,129.2$ (C-Ar), 129.2 (C C $^{-A r}$ ), 91.1 (C-1), 74.8 (C-5), 71.9 (C-3), 68.5 (C-2), 66.5 (C-4), 60.4 (C-6).

$3.0 \mathrm{~g}(6.09 \mathrm{mmol}, 1.0 \mathrm{eq})$ of tribenzoyl- $\alpha$-D-galactose $(12 \alpha-1)$ and $4.11 \mathrm{~g}(18.27 \mathrm{mmol}, 3.0 \mathrm{eq})$ of $\mathrm{N}$ iodosuccinimide were dissolved in $60 \mathrm{~mL}$ of absolute $\mathrm{N}, \mathrm{N}$-dimethylformamide and cooled to $0^{\circ} \mathrm{C} .6 .39 \mathrm{~g}$ 
(24.36 mmol, $4.0 \mathrm{eq}$ ) of triphenylphosphine were added over 20 minutes, and the reaction mixture was heated to $55^{\circ} \mathrm{C}$. After stirring for $24 \mathrm{~h}$ the reaction was stopped by adding of $15 \mathrm{~mL}$ of methanol. The organic layer was concentrated in vacuo $\left(60^{\circ} \mathrm{C}\right)$, diluted with dichloromethane, washed with water, dried $\left(\mathrm{MgSO}_{4}\right)$ and filtered. The filtrate was concentrated and the residue purified by flash chromatography $(5 \times 20 \mathrm{~cm}$, eluent: cyclohexane/ethyl acetate 9:1 $\rightarrow 6: 1$ ). Yield of $13 \alpha: 2.69 \mathrm{~g}$ (73.3\%). $\mathrm{R}_{\mathrm{f}}=0.40$ (cyclohexane/ethyl acetate $3: 1$ ). $[\alpha]_{D}{ }^{24}=-142.9^{\circ}$ (c = 0.50, dichloromethane). ESI/MS: $m / z 602.79[\mathrm{M}+\mathrm{H}]^{+}$, (calcd for $\left.\mathrm{C}_{27} \mathrm{H}_{24} \mathrm{lO} \mathrm{O}_{8}, 603.05\right) .{ }^{1} \mathrm{H}-$ NMR (270 MHz, [D 6 -DMSO): $\delta=8.11(\mathrm{~d}, 2 \mathrm{H}, J=7.3 \mathrm{~Hz}, \mathrm{H}-\mathrm{Ar}), 7.95$ (d, 2H, J=7.3 Hz, H-Ar), 7.79-7.71 (m, 3H, $\mathrm{H}-\mathrm{Ar}$ ), 7.60 (dd, 3H, J = 7.5 Hz, H-Ar), 7.58-7.52 (m, 1H, H-Ar), 7.47 (dd, 2H, J = 7.5 Hz, H-Ar), 7.37 (dd, $2 \mathrm{H}, J=$ $7.7 \mathrm{~Hz}, \mathrm{H}-\mathrm{Ar}$ ), $6.64\left(\mathrm{~d}, 1 \mathrm{H}, J_{1,2}=3.4 \mathrm{~Hz}, \mathrm{H}-1\right), 5.97\left(\mathrm{~d}, 1 \mathrm{H}, J_{\mathrm{OH}-4,4}=5.6 \mathrm{~Hz}, \mathrm{OH}-4\right), 5.80\left(\mathrm{dd}, 1 \mathrm{H}, J_{1,2}=3.4 \mathrm{~Hz}\right.$, $\left.J_{2,3}=10.6 \mathrm{~Hz}, \mathrm{H}-2\right), 5.73\left(\mathrm{dd}, 1 \mathrm{H}, J_{2,3}=10.6 \mathrm{~Hz}, J_{3,4}=1.9 \mathrm{~Hz}, \mathrm{H}-3\right.$ ), 4.53 (ddd, $1 \mathrm{H}, J_{3,4}=1.9 \mathrm{~Hz}, J_{4,5}=J_{\mathrm{OH}-4,4}=5.5$ $\mathrm{Hz}, \mathrm{H}-4), 4.45-4.41(\mathrm{~m}, 1 \mathrm{H}, \mathrm{H}-5), 3.43\left(\mathrm{dd}, 1 \mathrm{H}, J_{5,6 \mathrm{a}}=6.1 \mathrm{~Hz}, J_{6 a, 6 b}=10.0 \mathrm{~Hz}, \mathrm{H}-6 \mathrm{a}\right), 3.32\left(\mathrm{dd}, 1 \mathrm{H}, J_{5,6 b}=7.7 \mathrm{~Hz}\right.$, $\left.J_{6 a, 6 b}=10.0 \mathrm{~Hz}, \mathrm{H}-6 \mathrm{~b}\right) .{ }^{13} \mathrm{C}-\mathrm{NMR}\left(68 \mathrm{MHz},\left[\mathrm{D}_{6}\right]-\mathrm{DMSO}\right): \delta=165.8,165.5,164.8$ (CO-Bz), 134.7, 134.3. $134.1\left(\mathrm{C}_{p^{-}}\right.$ Ar), 130.2, 129.9 (C-Ar), 129.8 (C C $_{-}$Ar), 129.6, 129.5, 129.3 (C-Ar), 129.2 (Ci-Ar), 90.8 (C-1), 74.7 (C-5), 71.6 (C-3), 68.0 (C-2), 67.5 (C-4), 3.8 (C-6).

\section{1,2,3-Tri-O-benzoyl-6-deoxy-6-iodo- $\beta$-D-galactopyranose (13 $\beta$ ). Tribenzoyl-4,6-O-benzylidene- $\beta$-D-galactose} $12 \beta$ (5.0 g, $8.6 \mathrm{mmol}, 1.0 \mathrm{eq})$ was dissolved in acetonitrile $(50 \mathrm{~mL})$ and methanol $(50 \mathrm{~mL})$ and heated to $80{ }^{\circ} \mathrm{C}$. Hydrochloric acid $(5.0 \mathrm{~mL}, 2 \mathrm{~N})$ was added dropwise and the reaction was stirred under reflux for $2 \mathrm{~h}$. The reaction was stopped by adding triethylamine $(4.2 \mathrm{~mL})$. The organic layer was concentrated in vacuo, diluted with dichloromethane, washed with water, dried $\left(\mathrm{MgSO}_{4}\right)$ and filtered. The filtrate was concentrated in vacuo and the residue purified by flash chromatography $(5 \times 25 \mathrm{~cm}$, eluent: cyclohexane/ethyl acetate $6: 1 \rightarrow 1: 1)$. Yield of 1,2,3-tri-O-benzoyl- $\beta$-D-galactopyranose (12 $\beta-1)$ : $3.44 \mathrm{~g}(81.2 \%)$. $\mathrm{R}_{\mathrm{f}}=0.57$ (cyclohexane/acetone 1:1). $[\alpha]_{D}{ }^{26}=-247.2^{\circ}$ (c = 0.54, dichloromethane). ${ }^{1} \mathrm{H}-\mathrm{NMR}\left(270 \mathrm{MHz},\left[\mathrm{D}_{6}\right]-\mathrm{DMSO}\right): \delta=7.91-7.82(\mathrm{~m}, 6 \mathrm{H}, \mathrm{H}-\mathrm{Ar}), 7.70-$ 7.38 (m, 9H, H-Ar), 6.28 (d, $\left.1 \mathrm{H}, J_{1,2}=8.4 \mathrm{~Hz}, \mathrm{H}-1\right), 5.84$ (dd, $\left.1 \mathrm{H}, J_{1,2}=8.4 \mathrm{~Hz}, J_{2,3}=10.2 \mathrm{~Hz}, \mathrm{H}-2\right), 5.59\left(\mathrm{~d}, 1 \mathrm{H}, J_{\mathrm{OH}-}\right.$ $4,4=5.5 \mathrm{~Hz}, \mathrm{OH}-4), 5.53\left(\mathrm{dd}, 1 \mathrm{H}, J_{2,3}=10.2 \mathrm{~Hz}, J_{3,4}=3.0 \mathrm{~Hz}, \mathrm{H}-3\right), 4.88\left(\mathrm{t}, 1 \mathrm{H}, \mathrm{J}_{\mathrm{OH}-6,6}=5.7 \mathrm{~Hz}, \mathrm{OH}-6\right), 4.27(\mathrm{dd}, 1 \mathrm{H}$, $\left.J_{\mathrm{OH}-4,4}=5.5 \mathrm{~Hz}, J_{3,4}=3.0 \mathrm{~Hz}, \mathrm{H}-4\right), 4.05\left(\mathrm{dd}, 1 \mathrm{H}, J_{5,6}=8.6 \mathrm{~Hz}, \mathrm{H}-5\right), 3.70-3.52(\mathrm{~m}, 2 \mathrm{H}, \mathrm{H}-6 \mathrm{a}, \mathrm{H}-6 \mathrm{~b}) .{ }^{13} \mathrm{C}-\mathrm{NMR}(68$ $\mathrm{MHz},\left[\mathrm{D}_{6}\right]$-DMSO): $\delta=165.7,164.6$ (CO-Bz), 134.7, 134.3, 134.1 (Cp-Ar), 129.9, 129.8, 129.6, 129.4 (C-Ar), 129.3 (Ci-Ar), 129.2 (C-Ar), 128.8 (Ci-Ar), 93.2 (C-1), 76.7 (C-5), 74.8 (C-3), 69.9 (C-2), 65.9 (C-4), 60.1 (C-6).

Tribenzoyl- $\beta$-D-galactose $(\mathbf{1 2} \beta-\mathbf{1})(6.88 \mathrm{~g}, 14.0 \mathrm{mmol}, 1.0 \mathrm{eq})$ and $N$-iodosuccinimide $(9.53 \mathrm{~g}, 42.3 \mathrm{mmol}$, $3.0 \mathrm{eq})$ were dissolved in absolute $\mathrm{N}, \mathrm{N}$-dimethylformamide $(140 \mathrm{~mL})$ and cooled to $0^{\circ} \mathrm{C}$. Triphenylphosphine $(11.0 \mathrm{~g}, 41.9 \mathrm{mmol}, 3.0 \mathrm{eq})$ was added over 20 minutes and the reaction mixture was heated to $65^{\circ} \mathrm{C}$. After stirring for $18 \mathrm{~h}$ the reaction was stopped by adding methanol $(15 \mathrm{~mL})$. The organic layer was concentrated in vacuo $\left(60^{\circ} \mathrm{C}\right)$, the residue diluted with dichloromethane, washed with water, dried $\left(\mathrm{MgSO}_{4}\right)$ and filtered. The filtrate was concentrated in vacuo and the residue purified by flash chromatography $(7.5 \times 18 \mathrm{~cm}$, eluent: cyclohexane/ethyl acetate 9:1 $\rightarrow 3: 1$ ). Yield of $13 \beta: 7.12 \mathrm{~g}(85 \%)$. $R_{f}=0.50$ (cyclohexane/ethyl acetate $3: 1$ ). $[\alpha]_{D}{ }^{28}=-238.3^{\circ}$ (c = 0.50, dichloromethane). ${ }^{1} \mathrm{H}-\mathrm{NMR}\left(270 \mathrm{MHz},\left[\mathrm{D}_{6}\right]-\mathrm{DMSO}\right): \delta=7.93-7.80(\mathrm{~m}, 6 \mathrm{H}, \mathrm{H}-\mathrm{Ar}), 7.70-$ $7.39(\mathrm{~m}, 9 \mathrm{H}, \mathrm{H}-\mathrm{Ar}), 6.31$ (d, $\left.1 \mathrm{H}, J_{1,2}=8.2 \mathrm{~Hz}, \mathrm{H}-1\right), 5.88\left(\mathrm{~d}, 1 \mathrm{H}, \mathrm{J}_{\mathrm{OH}-4,4}=5.5 \mathrm{~Hz}, \mathrm{OH}-4\right), 5.81\left(\mathrm{dd}, 1 \mathrm{H}, J_{1,2}=8.2 \mathrm{~Hz}\right.$, $\left.J_{2,3}=10.3 \mathrm{~Hz}, \mathrm{H}-2\right), 5.57$ (dd, $\left.1 \mathrm{H}, J_{2,3}=10.3 \mathrm{~Hz}, J_{3,4}=2.8 \mathrm{~Hz}, \mathrm{H}-3\right), 4.41$ (ddd, $1 \mathrm{H}, J_{3,4}=2.8 \mathrm{~Hz}, J_{\mathrm{OH}-4,4}=J_{4,5}=5.5$ $\mathrm{Hz}, \mathrm{H}-4), 4.35(\mathrm{~m}, 1 \mathrm{H}, \mathrm{H}-5), 3.47$ (dd, $\left.1 \mathrm{H}, J_{5,6 a}=6.6 \mathrm{~Hz}, J_{6 a, 6 b}=10.1 \mathrm{~Hz}, \mathrm{H}-6 \mathrm{a}\right), 3.30\left(\mathrm{dd}, 1 \mathrm{H}, J_{5,6 b}=7.3 \mathrm{~Hz}, J_{6 a, 6 b}=\right.$ $10.1 \mathrm{~Hz}, \mathrm{H}-6 \mathrm{~b}) .{ }^{13} \mathrm{C}-\mathrm{NMR}$ (68 MHz, [D $]$-DMSO): $\delta=165.7,164.5$ (CO-Bz), 134.8, 134.3, $134.1\left(\mathrm{C}_{p}-\mathrm{Ar}\right), 129.9$, 129.8 (C-Ar), 129.7 ( $\left.C_{i}-A r\right), 129.6,129.4,129.2$ (C-Ar), 129.2 (Ci-Ar), 92.9 (C-1), 76.4 (C-5), 74.5 (C-3), 69.5 (C-2), 66.8 (C-4), 3.5 (C-6).

1,2,3-Tri-O-benzoyl-6-deoxy- $\alpha$-D-galactopyranose (14 $\alpha)$. Pd/C (10\% Pd, $8.73 \mathrm{~g}, 8.22 \mathrm{mmol})$ was suspended in absolute methanol $(150 \mathrm{~mL})$ containing acetic acid $(98 \%, 17 \mathrm{~mL})$ and stirred for $2 \mathrm{~h}$ under a hydrogen atmosphere. lodide $13 \alpha(3.42 \mathrm{~g}, 5.68 \mathrm{mmol})$ dissolved in absolute methanol $(20 \mathrm{~mL})$ was added and the 
reaction mixture was stirred for $2 \mathrm{~d}$ under a hydrogen atmosphere. Subsequently, the catalyst was removed by filtration over Celite and washed extensively with dichloromethane. The combined organic layers were extracted with saturated potassium hydrogen carbonate and water, dried $\left(\mathrm{MgSO}_{4}\right)$ and filtered. The filtrate was concentrated in vacuo and the residue purified by flash chromatography $(5 \times 18 \mathrm{~cm}$, eluent: cyclohexane/ethyl acetate $8: 1 \rightarrow 7: 1 \rightarrow 6: 1 \rightarrow 4: 1)$. $1.02 \mathrm{~g}(29.8 \%)$ of the starting material $13 \alpha$ were recovered. Yield: $1.51 \mathrm{~g}(55.9 \%) . R_{f}=0.22$ (cyclohexane/ethyl acetate $\left.4: 1\right) .[\alpha]_{D}{ }^{24}=+155.0^{\circ}(c=0.50$, dichloromethane). ESI/MS: $m / z$ 477.12 $[\mathrm{M}+\mathrm{H}]^{+}$(calcd for $\left.\mathrm{C}_{27} \mathrm{H}_{25} \mathrm{O}_{8}, 477.15\right)$. ${ }^{1} \mathrm{H}-\mathrm{NMR}\left(360 \mathrm{MHz},\left[\mathrm{D}_{6}\right]-\right.$ DMSO): $\delta=8.07$ (d, 2H, J = 7.5 Hz, H-Ar), 7.93 (d, 2H, J= 7.5 Hz, H-Ar), 7.75-7.70 (m, 3H, H-Ar), 7.65-7.57 (m, $3 \mathrm{H}, \mathrm{H}-\mathrm{Ar}$ ), 7.53 (dd, 2H, J=7.5 Hz, H-Ar), 7.47 (dd, 2H, J= $7.9 \mathrm{~Hz}, \mathrm{H}-\mathrm{Ar}$ ), 7.36 (dd, 2H, J = 7.9 Hz, H-Ar), 6.58 (d, $\left.1 \mathrm{H}, J_{1,2}=3.2 \mathrm{~Hz}, \mathrm{H}-1\right), 5.78-5.70(\mathrm{~m}, 3 \mathrm{H}, \mathrm{H}-2, \mathrm{H}-3, \mathrm{OH}-4), 4.43\left(\mathrm{dq}, 1 \mathrm{H}, J_{4,5}<1.0 \mathrm{~Hz}, J_{5,6}=6.2 \mathrm{~Hz}, \mathrm{H}-5\right), 4.15-4.12$ (m, 1H, H-4), 1.22 (d, 3H, J5,6 = $6.2 \mathrm{~Hz}, \mathrm{H}-6) .{ }^{13} \mathrm{C}-\mathrm{NMR}$ (90 MHz, [D $\left.]-\mathrm{DMSO}\right): \delta=165.3,165.0,164.4$ (CO-Bz), 134.0, 133.7, 133.5 ( $\left.C_{p}-A r\right), 129.5$ (C-Ar), 129.3 ( $\left.C_{i}-A r\right), 129.0$ (C-Ar), 129.0 ( $\left.C_{i}-A r\right), 128.7$ (C-Ar), 128.6 (Ci-Ar), 90.7 (C-1), 71.2 (C-3), 69.5 (C-5), 68.7 (C-4), 67.7 (C-2), 16.2 (C-6).

1,2,3-Tri-O-benzoyl-6-deoxy- $\beta$-D-galactopyranose (14ß). Pd/C-catalyst (10\% Pd, $11.2 \mathrm{~g}, 10.6 \mathrm{mmol})$ was suspended in absolute methanol $(155 \mathrm{~mL})$ and acetic acid $(98 \%, 16 \mathrm{~mL})$ and stirred for $2 \mathrm{~h}$ under a hydrogen atmosphere. A solution of iodide $13 \beta(3.1 \mathrm{~g}, 5.15 \mathrm{mmol})$ in absolute methanol $(20 \mathrm{~mL})$ was added. After $2 \mathrm{~d}$ the catalyst was removed by filtration over Celite and repeatedly washed with dichloromethane. The combined organic layer was extracted with saturated potassium hydrogen carbonate and water, dried $\left(\mathrm{MgSO}_{4}\right)$ and concentrated in vacuo. The residue was purified by flash chromatography $(5 \times 20 \mathrm{~cm}$, eluent: cyclohexane/ethyl acetate $8: 1 \rightarrow 5: 1) .1 .9 \mathrm{~g}(61.3 \%)$ of the starting material $13 \beta$ were recovered. Yield: $867 \mathrm{mg}$ (35.3\%). $R_{f}=0.34$ (cyclohexane/ethyl acetate 4:1). ${ }^{1} \mathrm{H}-\mathrm{NMR}\left(270 \mathrm{MHz},\left[\mathrm{D}_{6}\right]-\mathrm{DMSO}\right): \delta=7.91-7.82(\mathrm{~m}, 5 \mathrm{H}, \mathrm{H}-\mathrm{Ar})$, 7.70-7.35 (m, 10H, H-Ar), 6.22 (d, $\left.1 \mathrm{H}, J_{1,2}=8.1 \mathrm{~Hz}, \mathrm{H}-1\right), 5.79\left(\mathrm{dd}, 1 \mathrm{H}, J_{1,2}=8.1 \mathrm{~Hz}, J_{2,3}=10.3 \mathrm{~Hz}, \mathrm{H}-2\right), 5.62(\mathrm{~d}$, $\left.1 \mathrm{H}, J_{\mathrm{OH}-4,4}=5.8 \mathrm{~Hz}, \mathrm{OH}-4\right), 5.53\left(\mathrm{dd}, 1 \mathrm{H}, J_{2,3}=10.3 \mathrm{~Hz}, J_{3,4}=3.0 \mathrm{~Hz}, \mathrm{H}-3\right), 4.21\left(\mathrm{dq}, 1 \mathrm{H}, J_{4,5}<1.0 \mathrm{~Hz}, 5,6=6.2 \mathrm{~Hz}\right.$, $\mathrm{H}-5)$, 4.03-4.00 (m, 1H, H-4), 1.25 (d, 3H, $\left.J_{5,6}=6.2 \mathrm{~Hz}, \mathrm{H}-6\right) .{ }^{13} \mathrm{C}-\mathrm{NMR}$ (68 MHz, [D6]-DMSO): $\delta=165.7,165.6$, 164.7 (CO-Bz), 134.7, 134.3, 134.1 ( $C_{p}-\mathrm{Ar}$ ), 129.9, 129.8 (C-Ar), 129.6 (Ci-Ar), 129.4 (C-Ar), 129.0 (Ci-Ar), 93.2 (C1), 74.8 (C-3), 71.8 (C-5), 69.6 (C-2), 68.8 (C-4), 16.7 (C-6).

4-Azido-1,2,3-tri-O-benzoyl-4,6-dideoxy- $\alpha$-D-glucopyranose (15 $\alpha)$. 6-Deoxysugar $14 \alpha \quad(7.3 \mathrm{~g}, 15.3 \mathrm{mmol}$, $1.0 \mathrm{eq})$ was dissolved in absolute dichloromethane $(112 \mathrm{~mL})$ and absolute pyridine $(19 \mathrm{~mL}, 236 \mathrm{mmol})$ and cooled to $-18{ }^{\circ} \mathrm{C}$. Trifluoromethanesulfonic anhydride $(6.2 \mathrm{~mL}, 37.6 \mathrm{mmol}, 2.4 \mathrm{eq})$ was added. After stirring for 110 minutes the solution was extracted with water, saturated potassium hydrogen carbonate $(2 \mathrm{x})$ and $2 \mathrm{~N}$ hydrochloric acid. The organic layer was dried $\left(\mathrm{MgSO}_{4}\right)$ and filtered. The filtrate was concentrated and dried in high vacuum. Subsequently, sodium azide $(9.0 \mathrm{~g}, 138 \mathrm{mmol}, 8.5 \mathrm{eq})$ was added to the crude triflate. The mixture was suspended in absolute $N, N$-dimethylformamide $(240 \mathrm{~mL})$ and stirred for 30 minutes at room temperature. The organic layer was concentrated in vacuo $\left(60{ }^{\circ} \mathrm{C}\right)$ and the residue was diluted with dichloromethane $(300 \mathrm{~mL})$. The solution was extracted with water, dried over $\mathrm{MgSO}_{4}$ and filtered. The filtrate was concentrated in vacuo and the residue purified by flash chromatography $(7.5 \times 17 \mathrm{~cm}$, eluent: cyclohexane/ethyl acetate $12: 1 \rightarrow 9: 1)$. Yield: $7.05 \mathrm{~g}$ (92\% over 2 steps). $\mathrm{R}_{\mathrm{f}}=0.47$ (cyclohexane/acetone 3:1). $[\alpha]_{D}{ }^{25}=+213.2^{\circ}$ (c = 0.55, dichloromethane). ESI-MS m/z $523.73[\mathrm{M}+\mathrm{Na}]^{+}$(calcd for $\left.\mathrm{C}_{27} \mathrm{H}_{23} \mathrm{~N}_{3} \mathrm{O}{ }_{7} \mathrm{Na}, 524.14\right) .{ }^{1} \mathrm{H}-$ NMR (270 MHz, [D $]$-DMSO): $\delta=8.06(\mathrm{~d}, 2 \mathrm{H}, J=7.1 \mathrm{~Hz}, \mathrm{H}-\mathrm{Ar}), 7.97$ (d, 2H, J= 7.3 Hz, H-Ar), 7.79-7.49 (m, 9H, $\mathrm{H}-\mathrm{Ar}$ ), 7.39 (dd, $2 \mathrm{H}, \mathrm{J}=7.7 \mathrm{~Hz}, \mathrm{H}-\mathrm{Ar}), 6.64\left(\mathrm{~d}, 1 \mathrm{H}, J_{1,2}=3.6 \mathrm{~Hz}, \mathrm{H}-1\right), 5.87$ (dd, $\left.1 \mathrm{H}, J_{2,3}=J_{3,4}=10.1 \mathrm{~Hz}, \mathrm{H}-3\right), 5.54$ $\left(\mathrm{dd}, 1 \mathrm{H}, J_{1,2}=3.6 \mathrm{~Hz}, J_{2,3}=10.1 \mathrm{~Hz}, \mathrm{H}-2\right), 4.27\left(\mathrm{dd}, 1 \mathrm{H}, J_{3,4}=J_{4,5}=10.1 \mathrm{~Hz}, \mathrm{H}-4\right), 4.14-4.06(\mathrm{~m}, 1 \mathrm{H}, \mathrm{H}-5), 1.38(\mathrm{~d}$, $\left.3 \mathrm{H}, J_{5,6}=6.0 \mathrm{~Hz}, \mathrm{H}-6\right) .{ }^{13} \mathrm{C}-\mathrm{NMR}\left(68 \mathrm{MHz},\left[\mathrm{D}_{6}\right]-\mathrm{DMSO}\right): \delta=165.7,165.2,164.2$ (CO-Bz), 134.8, 134.6, $134.5\left(\mathrm{C}_{p^{-}}\right.$ Ar), 130.1, 129.9, 129.7, 129.6, 129.5, 129.4 (C-Ar), 129.1, 129.0, 128.7 (Ci-Ar), 90.3 (C-1), 71.5 (C-3), 71.0 (C-2), 69.5 (C-5), 64.4 (C-4), 18.5 (C-6). 
4-Azido-1,2,3-tri-O-benzoyl-4,6-dideoxy- $\beta$-D-glucopyranose (15 $\beta$ ). Under an inert gas 6-deoxysugar $\mathbf{1 4} \boldsymbol{\beta}$ $(3.86 \mathrm{~g}, 8.1 \mathrm{mmol}, 1.0 \mathrm{eq})$ was dissolved in absolute dichloromethane $(60 \mathrm{~mL})$ and absolute pyridine $(10 \mathrm{~mL}$, $124 \mathrm{mmol}$ ) and cooled to $-18{ }^{\circ} \mathrm{C}$. Trifluoromethanesulfonic anhydride $(3.0 \mathrm{~mL}, 24.3 \mathrm{mmol}, 2.2 \mathrm{eq})$ was added. After stirring for 60 minutes the solution was extracted with water, saturated potassium hydrogen carbonate $(2 \mathrm{x})$ and hydrochloric acid $(2 \mathrm{~N})$. The organic layer was dried $\left(\mathrm{MgSO}_{4}\right)$ and filtered. The filtrate was concentrated and dried in high vacuum. Subsequently, sodium azide $(5.26 \mathrm{~g}, 81 \mathrm{mmol}, 10 \mathrm{eq})$ was added to the crude triflate. The mixture was suspended in absolute $N, N$-dimethylformamide $(116 \mathrm{~mL})$ and stirred for 30 minutes at room temperature. The organic layer was concentrated in vacuo $\left(60^{\circ} \mathrm{C}\right)$ and the residue was diluted with $300 \mathrm{~mL}$ of dichloromethane. The solution was extracted with water, dried over $\mathrm{MgSO}_{4}$ and filtered. The filtrate was concentrated in vacuo and the residue purified by flash chromatography $(5 \times 16 \mathrm{~cm}$, eluent: cyclohexane/ethyl acetate $12: 1 \rightarrow 9: 1)$. Yield: $3.41 \mathrm{~g}$ (84\% over 2 steps). $\mathrm{R}_{\mathrm{f}}=0.34$ (cyclohexane/acetone 3:1). $[\alpha]_{D}{ }^{26}=-27.4^{\circ}$ (c = 0.48, dichloromethane). ${ }^{1} \mathrm{H}-\mathrm{NMR}\left(270 \mathrm{MHz},\left[\mathrm{D}_{6}\right]-\right.$ DMSO): $\delta=7.96-7.87$ (m, 4H, H-Ar), 7.79 (d, 2H, J = 7.3 Hz, H-Ar), 7.70-7.40 (m, 9H, H-Ar), 6.37 (d, $1 \mathrm{H}, J_{1,2}=8.1$ $\mathrm{Hz}, \mathrm{H}-1), 5.94\left(\mathrm{dd}, 1 \mathrm{H}, J_{2,3}=J_{3,4}=9.5 \mathrm{~Hz}, \mathrm{H}-3\right), 5.54\left(\mathrm{dd}, 1 \mathrm{H}, J_{2,3}=9.5 \mathrm{~Hz}, J_{1,2}=8.1 \mathrm{~Hz}, \mathrm{H}-2\right), 4.10-4.05(\mathrm{~m}, 2 \mathrm{H}, \mathrm{H}-$ 4, H-5), 1.36 (d, 3H, J5,6 = 5.1 Hz, H-6). ${ }^{13} \mathrm{C}-\mathrm{NMR}\left(68 \mathrm{MHz},\left[\mathrm{D}_{6}\right]-\mathrm{DMSO}\right): \delta=165.6,165.4,164.5$ (CO-Bz), 134.5, 134.4, 134.3 (C $c_{p}$-Ar), 129.9, 129.8, 129.6, 129.5, 129.4 (C-Ar), 129.1, 128.9, 128.6 (Ci-Ar), 92.5 (C-1), 73.4 (C-3), 72.0 (C-2), 71.3 (C-5), 64.9 (C-4), 18.4 (C-6).

\section{4-Azido-2,3-di-O-benzoyl-4,6-dideoxy-D-glucopyranose (10a) and 4-azido-2,3-di-O-benzoyl-4,6-dideoxy- $\alpha$-D-} glucopyranosyl-trichloroacetimidate (10) from $15 \alpha$. Azide $15 \alpha(1.32 \mathrm{~g}, 2.63 \mathrm{mmol}, 1.0 \mathrm{eq})$ was dissolved in absolute $\mathrm{N}, \mathrm{N}$-dimethylformamide $(12 \mathrm{~mL})$. Hydrazine acetate $(2.42 \mathrm{~g}, 26.3 \mathrm{mmol}, 10 \mathrm{eq})$ was added and the suspension was stirred at room temperature. After $5.5 \mathrm{~h}$ the reaction was stopped by adding acetone $(10 \mathrm{~mL})$ and stirring was continued until the remaining solid hydrazine acetate was completely dissolved. The solution was diluted with dichloromethane $(200 \mathrm{~mL})$ and extracted with water and saturated potassium hydrogen carbonate. The organic layer was dried $\left(\mathrm{MgSO}_{4}\right)$ and filtered. The filtrate was concentrated in vacuo and purified by flash chromatography $(3.5 \times 28 \mathrm{~cm}$, eluent: cyclohexane/acetone 10:1). Yield of hemiacetal 10a: $886 \mathrm{mg}(84.8 \%, \alpha: \beta=2: 1) . R_{\mathrm{f}}=0.26$ (cyclohexane/acetone $2: 1$ ).

from 15 3 : Azide $15 \beta$ (3.40 g, $6.8 \mathrm{mmol}, 1.0 \mathrm{eq})$ was dissolved in absolute $N, N$-dimethylformamide $(18 \mathrm{~mL})$. Hydrazine acetate $(4.70 \mathrm{~g}, 50.9 \mathrm{mmol}, 7.5 \mathrm{eq})$ was added and the suspension was stirred at room temperature. After $3.5 \mathrm{~h}$ the reaction was stopped by adding acetone $(20 \mathrm{~mL})$ and stirring was continued until the remaining solid hydrazine acetate was completely dissolved. The solution was diluted with dichloromethane $(400 \mathrm{~mL})$ and extracted with water and saturated potassium hydrogen carbonate. The organic layer was dried $\left(\mathrm{MgSO}_{4}\right)$ and filtered. The filtrate was concentrated in vacuo and purified by flash chromatography $(5 \times 20 \mathrm{~cm}$, eluent: cyclohexane/acetone 10:1). Yield of hemiacetal 10a: $2.38 \mathrm{~g}(88.6 \%, \alpha: \beta=2: 1)$. ${ }^{1} \mathrm{H}-\mathrm{NMR}\left(270 \mathrm{MHz},\left[\mathrm{D}_{6}\right]-\right.$ DMSO): $\delta=7.96$ (d, 2H, J= $7.1 \mathrm{~Hz}, \mathrm{H}-\mathrm{Ar}$ ), 7.90 (d, 1H, J= $7.1 \mathrm{~Hz}, \mathrm{H}-\mathrm{Ar}$ ), 7.83 (d, 3H, J = 7.1 Hz, H-Ar), 7.66-7.57 (m, 3H, H-Ar), 7.51-7.43 (m, 6H, H-Ar), $7.28\left(\mathrm{~d}, 1 \mathrm{H}, \mathrm{J}_{\mathrm{OH}-1 \beta, 1}=6.2 \mathrm{~Hz}, \mathrm{OH}-1 \beta\right), 7.24\left(\mathrm{~d}, 1 \mathrm{H}, \mathrm{J}_{\mathrm{OH}-1 \alpha, 1}=3.9 \mathrm{~Hz}, \mathrm{OH}-\right.$ $1 \alpha), 5.75\left(\mathrm{dd}, 1 \mathrm{H}, J_{2 \alpha, 3 \alpha}=J_{3 \alpha, 4 \alpha}=9.6 \mathrm{~Hz}, \mathrm{H}-3 \alpha\right), 5.61\left(\mathrm{dd}, 1 \mathrm{H}, J_{2 \beta, 3 \beta}=J_{3 \beta, 4 \beta}=9.5 \mathrm{~Hz}, \mathrm{H}-3 \beta\right), 5.39\left(\mathrm{dd}, 1 \mathrm{H}, J_{\mathrm{OH}-1 \alpha, 1}=\right.$ $\left.J_{1,2}=3.6 \mathrm{~Hz}, \mathrm{H}-1 \alpha\right), 5.11\left(\mathrm{dd}, 1 \mathrm{H}, J_{1,2}=3.6 \mathrm{~Hz}, J_{2,3}=9.6 \mathrm{~Hz}, \mathrm{H}-2 \alpha\right), 5.12\left(\mathrm{dd}, 1 \mathrm{H}, J_{1,2}=7.6 \mathrm{~Hz}, J_{2,3}=9.6 \mathrm{~Hz}, \mathrm{H}-2 \beta\right)$, $5.02\left(\mathrm{dd}, 1 \mathrm{H}, J_{1,2}=7.6 \mathrm{~Hz}, J_{\mathrm{OH}-1 \beta, 1}=6.2 \mathrm{~Hz}, \mathrm{H}-1 \beta\right), 4.01-3.83(\mathrm{~m}, 3 \mathrm{H}, \mathrm{H}-5 \alpha, \mathrm{H}-4 \alpha, \mathrm{H}-4 \beta), 3.80-3.70(\mathrm{~m}, 1 \mathrm{H}, \mathrm{H}-5 \beta)$, $1.32\left(\mathrm{~d}, 3 \mathrm{H}, J_{5,6}=6.0 \mathrm{~Hz}, \mathrm{H}-6 \beta\right), 1.31\left(\mathrm{~d}, 3 \mathrm{H}, J_{5,6}=5.6 \mathrm{~Hz}, \mathrm{H}-6 \alpha\right) .{ }^{13} \mathrm{C}-\mathrm{NMR}\left(68 \mathrm{MHz},\left[\mathrm{D}_{6}\right]-\mathrm{DMSO}\right): \delta=165.9$, $165.6\left(C_{0}-B_{\alpha}\right), 165.7,165.3\left(C_{-1}-B z_{\beta}\right), 134.3\left(C_{p}-A_{\alpha}\right), 134.1\left(C_{p}-A_{\beta}\right), 129.8,129.7,129.4\left(C-\operatorname{Ar}_{\alpha}\right), 129.6,129.3$,

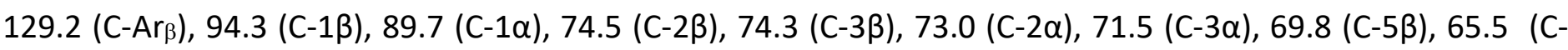

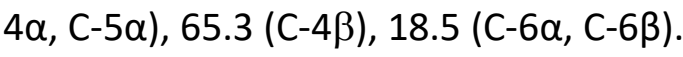

Hemiacetal 10a (1.08 g, $2.7 \mathrm{mmol}, 1.0 \mathrm{eq})$ was dissolved in absolute dichloromethane $(25 \mathrm{~mL})$ and cooled to $0^{\circ} \mathrm{C}$. Trichloroacetonitrile $(2.8 \mathrm{~mL}, 27.9 \mathrm{mmol}, 10.3 \mathrm{eq})$ followed by $\mathrm{DBU}(110 \mu \mathrm{L}, 0.74 \mathrm{mmol}, 0.35 \mathrm{eq})$ was 
added. After $2 \mathrm{~h}$ the solvent was concentrated in vacuo $\left(20{ }^{\circ} \mathrm{C}\right)$ and the residue purified by flash chromatography ( $3 \times 20 \mathrm{~cm}$, eluent: cyclohexane/acetone 15:1). Yield of imidate 10: $1.38 \mathrm{~g}(94.7 \%)$. $R_{f}=0.47$ (cyclohexane/acetone 3:1). $[\alpha]_{D}^{26}=+55.7^{\circ}$ (c=0.52, dichloromethane). ESI-MS $\mathrm{m} / z 562.92[\mathrm{M}+\mathrm{Na}]^{+}$(calcd for $\mathrm{C}_{22} \mathrm{H}_{19} \mathrm{Cl}_{3} \mathrm{~N}_{4} \mathrm{O}_{6} \mathrm{Na}$, (563.03). ${ }^{1} \mathrm{H}-\mathrm{NMR}\left(270 \mathrm{MHz},\left[\mathrm{D}_{6}\right]-\mathrm{DMSO}\right): \delta=9.91$ (s, $1 \mathrm{H}, \mathrm{NH}$ ), $7.96(\mathrm{~d}, 2 \mathrm{H}, \mathrm{J}=7.5 \mathrm{~Hz}, \mathrm{H}-\mathrm{Ar}$ ), $7.82(\mathrm{~d}, 2 \mathrm{H}, J=7.5 \mathrm{~Hz}, \mathrm{H}-\mathrm{Ar}$ ), 7.70-7.57 (m, 2H, H-Ar), 7.55-7.42 (m, 4H, H-Ar), 6.60 (bs, 1H, H-1), 5.81 (dd, 1H, $\left.J_{2,3}=J_{3,4}=10.0 \mathrm{~Hz}, \mathrm{H}-3\right), 5.51\left(\mathrm{dd}, 1 \mathrm{H}, J_{1,2}=3.0 \mathrm{~Hz}, J_{2,3}=10.0 \mathrm{~Hz}, \mathrm{H}-2\right), 4.23\left(\mathrm{dd}, 1 \mathrm{H}, J_{3,4}=J_{4,5}=10.0 \mathrm{~Hz}, \mathrm{H}-4\right)$, $3.94\left(\mathrm{dq}, 1 \mathrm{H}, J_{4,5}=10.0 \mathrm{~Hz}, J_{5,6}=6.2 \mathrm{~Hz}, \mathrm{H}-5\right), 1.38\left(\mathrm{~d}, 3 \mathrm{H}, J_{5,6}=6.2 \mathrm{~Hz}, \mathrm{H}-6\right) .{ }^{13} \mathrm{C}-\mathrm{NMR}\left(68 \mathrm{MHz},\left[\mathrm{D}_{6}\right]-\right.$ DMSO): $\delta=165.6,165.2$ (CO-Bz), 134.5, 134.3 (C $\left.p_{p}-A r\right), 129.9,129.8,129.4$ (C-Ar), 129.0, 128.7 (C $\left.o^{-}-A r\right), 94.3\left(C_{q^{-}}\right.$ $\mathrm{CCl}_{3}$ ), 92.9 (C-1), 71.2 (C-3), 70.9 (C-2), 69.4 (C-5), 64.3 (C-4), 18.2 (C-6).

5-Chloro-2-carboxymethylamino-benzoic acid (17). 4-Chloroanthranilic acid 16 (10.0 g, 58.3 mmol, 1.0 eq) was dissolved in $1 \mathrm{~N}$ sodium carbonate $(50 \mathrm{~mL})$ and the $\mathrm{pH}$ of the solution was adjusted to 8.5 with $1 \mathrm{~N}$ sodium carbonate. Chloroacetic acid $(7.2 \mathrm{~g}, 76.2 \mathrm{mmol}, 1.3 \mathrm{eq})$ was dissolved in $1 \mathrm{~N}$ sodium carbonate $(15 \mathrm{~mL})$ and adjusted to $\mathrm{pH} 3$ with $1 \mathrm{~N}$ sodium carbonate. Both solutions were united and adjusted to $\mathrm{pH} 9$ with $1 \mathrm{~N}$ sodium carbonate. The reaction was stirred under reflux and a $\mathrm{pH}$ of 9 was kept by periodical addition of $1 \mathrm{~N}$ sodium carbonate. After $6 \mathrm{~h}$ the reaction was cooled to room temperature and the solution was acidified to $\mathrm{pH} 4 \mathrm{with}$ $2 \mathrm{~N}$ hydrochloric acid. The resulting precipitate was left for $12 \mathrm{~h}$ at $3^{\circ} \mathrm{C}$ and filtered. The precipitate was briefly washed with a small amount of water and dried by lyophilization. The product was used for the next reaction without any purification. An aliquot was purified by flash chromatography for analytical purposes (eluent: toluene/ethyl acetate containing 1\% HOAc 7:1 $\rightarrow$ 5:1). Yield of 17: $8.77 \mathrm{~g}(65.5 \%)$. $\mathrm{R}_{\mathrm{f}}=0.34$ (cyclohexane/ethyl acetate 1:1 with 1.0\% HOAc (98\%). El/MS (70 eV): $\mathrm{m} / z(\%)=231$ (9) $\left[\left({ }^{37} \mathrm{Cl}\right) \mathrm{M}\right]^{+}, 229(27)\left[\left({ }^{35} \mathrm{Cl}\right) \mathrm{M}^{+}(\mathrm{calcd}\right.$ for $\left.\mathrm{C}_{9} \mathrm{H}_{8} \mathrm{CINO}_{4}, 229.01\right) .{ }^{1} \mathrm{H}-\mathrm{NMR}\left(270 \mathrm{MHz},\left[\mathrm{D}_{6}\right]-\mathrm{DMSO}\right): \delta=13.06$ (bs, $\left.2 \mathrm{H}, \mathrm{COOH}\right), 8.12$ (bs, $\left.1 \mathrm{H}, \mathrm{NH}\right), 7.73(\mathrm{~d}, 1 \mathrm{H}$, $\left.J_{4,6}=2.6 \mathrm{~Hz}, \mathrm{H}-6\right), 7.38\left(\mathrm{dd}, 1 \mathrm{H}, J_{3,4}=9.0 \mathrm{~Hz}, J_{4,6}=2.6 \mathrm{~Hz}, \mathrm{H}-4\right), 6.64\left(\mathrm{~d}, 1 \mathrm{H}, J_{3,4}=9.0 \mathrm{~Hz}, \mathrm{H}-3\right), 4.00(\mathrm{~s}, 2 \mathrm{H}, \mathrm{H}-7)$. ${ }^{13} \mathrm{C}-\mathrm{NMR}\left(68 \mathrm{MHz},\left[\mathrm{D}_{6}\right]-\mathrm{DMSO}\right): \delta=172.0,169.1(\mathrm{COOH}), 149.3\left(\mathrm{C}_{\mathrm{q}}-\mathrm{NH}\right), 134.5(\mathrm{C}-4), 131.0(\mathrm{C}-6), 118.6\left(\mathrm{C}_{\mathrm{q}}-\mathrm{Cl}\right)$, 114.2 (C-3), 112.1 ( $\left.\mathrm{C}_{\mathrm{q}}-\mathrm{COOH}\right), 44.8$ (C-7).

3-Acetoxy-5-chloroindol (18) via 1,3-diacetyl-5-chloroindol (17a). Compound 17 (8.77 g, $38.2 \mathrm{mmol}, 1.0 \mathrm{eq})$ was stirred in a mixture of acetic anhydride $(88 \mathrm{~mL}, 0.9 \mathrm{mmol})$ and sodium acetate $(9.39 \mathrm{~g}, 0.1 \mathrm{mmol})$ at $85^{\circ} \mathrm{C}$. After $3 \mathrm{~h}$ the reaction was cooled to room temperature and the precipitate was removed by filtration. The precipitate was washed with ethyl acetate $(300 \mathrm{~mL})$ and the combined filtrate was extracted with water $(3 \mathrm{x})$ and saturated potassium hydrogen carbonate $(2 \mathrm{x})$. The organic layer was dried $\left(\mathrm{MgSO}_{4}\right)$ and filtered. The filtrate was concentrated in vacuo and the residue purified by flash chromatography $(5 \times 20 \mathrm{~cm}$, eluent: cyclohexane/ethyl acetate 7:1 $\rightarrow$ 5:1). Yield of 1,3-diacetyl-5-chloroindol (17a): $6.09 \mathrm{~g}(63 \%) . \mathrm{R}_{\mathrm{f}}=0.64$ (toluene/ethyl acetate 5:1 with 1\% HOAc (98\%). El/MS (70 eV): $\mathrm{m} / z(\%)=253(7)\left[\left({ }^{37} \mathrm{Cl}\right) \mathrm{M}\right]^{+}, 251(20)\left[\left({ }^{35} \mathrm{Cl}\right) \mathrm{M}\right]^{+}$ (calcd for $\left.\mathrm{C}_{12} \mathrm{H}_{10} \mathrm{ClNO}_{3}, 251.03\right) .{ }^{1} \mathrm{H}-\mathrm{NMR}\left(360 \mathrm{MHz},\left[\mathrm{D}_{6}\right]-\mathrm{DMSO}\right): \delta=8.33\left(\mathrm{~d}, 1 \mathrm{H}, J_{6,7}=8.5 \mathrm{~Hz}, \mathrm{H}-7\right), 7.96(\mathrm{~s}, 1 \mathrm{H}$, $\mathrm{H}-2$ ), $7.63\left(\mathrm{~d}, 1 \mathrm{H}, J_{4,6}=2.1 \mathrm{~Hz}, \mathrm{H}-4\right), 7.40\left(\mathrm{dd}, 1 \mathrm{H}, J_{4,6}=2.1 \mathrm{~Hz}, J_{6,7}=8.5 \mathrm{~Hz}, \mathrm{H}-6\right), 2.60(\mathrm{~s}, 3 \mathrm{H}, \mathrm{NAc}), 2.37(\mathrm{~s}, 3 \mathrm{H}$, OAc). ${ }^{13} \mathrm{C}-N M R\left(90 \mathrm{MHz},\left[\mathrm{D}_{6}\right]-\mathrm{DMSO}\right): \delta=169.5,168.4$ (CO-Ac), 132.6 (C $\left.\mathrm{q}_{\mathrm{q}}-7 \mathrm{a}\right), 131.1\left(\mathrm{C}_{\mathrm{q}}-3\right), 128.0\left(\mathrm{C}_{\mathrm{q}}-5\right), 125.5$ (C-7), 125.2 ( $\left.\mathrm{C}_{\mathrm{q}}-3 \mathrm{a}\right), 117.6,117.5$ (C-2, C-4, C-6), 23.6 (NAc), 20.5 (OAc).

Under an inert gas 1,3-diacetyl-5-chloroindole 17a (1.0 g, $4.0 \mathrm{mmol}, 1.0 \mathrm{eq})$ was dissolved in sodium hydroxide $(50 \mathrm{~mL}, 5 \%)$ and stirred under reflux. After 30 minutes the reaction was cooled to $0^{\circ} \mathrm{C}$ and $7 \mathrm{~mL}(74 \mathrm{mmol}$, $18.5 \mathrm{eq}$ ) of acetic anhydride were added dropwise. The reaction was stirred for 30 minutes. The resulting white-blue precipitate was filtered and washed with water. The precipitate was dissolved in dichloromethane and the insoluble blue material was removed by filtration. The filtrate was concentrated and dried in high vacuo. Yield of 18: $0.73 \mathrm{~g}(87.1 \%) . \mathrm{R}_{\mathrm{f}}=0.40$ (toluene/ethyl acetate 7:1). El/MS (70 eV): $\mathrm{m} / z(\%)=211(7)$ $\left[\left({ }^{37} \mathrm{Cl}\right) \mathrm{M}\right]^{+}, 209(22)\left[\left({ }^{35} \mathrm{Cl}\right) \mathrm{M}\right]^{+}$(calcd for $\left.\mathrm{C}_{10} \mathrm{H}_{8} \mathrm{ClNO}_{2}, 209.02\right) .{ }^{1} \mathrm{H}-\mathrm{NMR}\left(270 \mathrm{MHz},\left[\mathrm{D}_{6}\right]-\mathrm{DMSO}\right): \delta=11.18$ (bs, $1 \mathrm{H}$, 
$\mathrm{NH}), 7.44\left(\mathrm{~d}, 1 \mathrm{H}, \mathrm{J}_{4,6}=1.7 \mathrm{~Hz}, \mathrm{H}-4\right), 7.41(\mathrm{~s}, 1 \mathrm{H}, \mathrm{H}-2), 7.38\left(\mathrm{~d}, 1 \mathrm{H}, J_{6,7}=8.8 \mathrm{~Hz}, \mathrm{H}-7\right), 7.11\left(\mathrm{dd}, 1 \mathrm{H}, J_{4,6}=1.7 \mathrm{~Hz}\right.$, $\left.J_{6,7}=8.8 \mathrm{~Hz}, \mathrm{H}-6\right), 2.31(\mathrm{~s}, 3 \mathrm{H}, \mathrm{Ac}) .{ }^{13} \mathrm{C}-\mathrm{NMR}\left(68 \mathrm{MHz},\left[\mathrm{D}_{6}\right]-\mathrm{DMSO}\right): \delta=169.3$ (CO-Ac), $132.3\left(\mathrm{C}_{\mathrm{q}^{-}}-7 \mathrm{a}\right), 129.1\left(\mathrm{C}_{\mathrm{q}^{-}}\right.$ 3), 124.2 ( $\left.\mathrm{C}_{\mathrm{q}}-5\right), 122.3$ (C-6), 121.3 ( $\left.\mathrm{C}_{\mathrm{q}}-3 \mathrm{a}\right), 117.1,116.9$ (C-2, C-4), 114.1 (C-7), 21.1 (Ac).

5,5'-Dichloroindigo (19). Compound $18(294 \mathrm{mg}, 1.40 \mathrm{mmol}, 0.5 \mathrm{eq})$ was dissolved in methanol (15 mL) and sodium hydroxide $(1 \mathrm{~mL}, 1 \mathrm{~N})$ was added. Immediately, compressed air was bubbled through the solution. After 15 minutes the blue precipitate was filtered, washed with water $(250 \mathrm{~mL})$, methanol $(200 \mathrm{~mL})$ and diethyl ether $(200 \mathrm{~mL})$. The residue was dried in a compartment dryer at $70^{\circ} \mathrm{C}$. Yield: $168 \mathrm{mg}(72.9 \%)$, blue solid. El/MS (70 eV): $\mathrm{m} / z(\%)=332(67)\left[\left({ }^{37} \mathrm{Cl}\right) \mathrm{M}\right]^{+}, 330(100)\left[\left({ }^{35} \mathrm{Cl}\right) \mathrm{M}\right]^{+}$(calcd for $\left.\mathrm{C}_{16} \mathrm{H}_{8} \mathrm{Cl}_{2} \mathrm{~N}_{2} \mathrm{O}_{2}, 330.00\right)$. For solution NMR 5,5'-dichloroindigo 19 was converted to its vat indigo ${ }^{31}$ form $19 \mathrm{v}$ by heating a mixture of 19, sodium dithionite and potassium hydroxide in $\mathrm{D}_{2} \mathrm{O}$. NMR of vat indigo 19v: ${ }^{1} \mathrm{H}-\mathrm{NMR}\left(270 \mathrm{MHz}, \mathrm{D}_{2} \mathrm{O}\right): \delta=7.53$ $\left(\mathrm{d}, 2 \mathrm{H}, J_{4,6}=1.9 \mathrm{~Hz}, \mathrm{H}-4\right), 7.26\left(\mathrm{~d}, 2 \mathrm{H}, J_{6,7}=8.6 \mathrm{~Hz}, \mathrm{H}-7\right), 6.99$ (dd, $\left.2 \mathrm{H}, J_{4,6}=1.9 \mathrm{~Hz}, J_{6,7}=8.6 \mathrm{~Hz}, \mathrm{H}-6\right) .{ }^{13} \mathrm{C}-\mathrm{NMR}$ $\left(68 \mathrm{MHz}, \mathrm{D}_{2} \mathrm{O}\right): \delta=138.9\left(\mathrm{C}_{\mathrm{q}}-7 \mathrm{a}\right), 132.7\left(\mathrm{C}_{\mathrm{q}}-3 \mathrm{a}\right), 124.3\left(\mathrm{C}_{\mathrm{q}}-2\right), 122.8\left(\mathrm{C}_{\mathrm{q}^{-}}-5\right), 120.3(\mathrm{C}-6), 116.5(\mathrm{C}-4), 116.3\left(\mathrm{C}_{\mathrm{q}}-3\right)$, 112.1 (C-7).

N-Benzyl-5,5'-dichloroindigo (11). 5,5'-Dichloroindigo 19 (2.06 g, $6.21 \mathrm{mmol}, 1.0 \mathrm{eq})$ was suspended in absolute $\mathrm{N}, \mathrm{N}$-dimethylformamide $(50 \mathrm{~mL}$ ), $\mathrm{NaH}$ ( $60 \%$ in oil suspension, $273 \mathrm{mg}, 6.83 \mathrm{mmol}, 1.1$ eq) was added and the suspension was stirred at room temperature. After 30 minutes a second portion of $\mathrm{NaH}(60 \%$ in oil suspension, $273 \mathrm{mg}, 6.83 \mathrm{mmol}, 1.1 \mathrm{eq})$ was added. After $2 \mathrm{~h}$ benzylbromide $(0.9 \mathrm{~mL}, 7.57 \mathrm{mmol}, 1.2 \mathrm{eq})$ was added dropwise. The reaction was stirred for $1 \mathrm{~h}$ at room temperature and was subsequently concentrated in vacuo $\left(60^{\circ} \mathrm{C}\right)$. The oily residue was dissolved in dichloromethane $(150 \mathrm{~mL})$ and extracted with water $(3 \mathrm{x})$. The organic layer was dried $\left(\mathrm{MgSO}_{4}\right)$ and filtered. The filtrate was concentrated in vacuo and the residue purified by flash chromatography $\left(6 \times 16 \mathrm{~cm}\right.$, eluent: toluene). Yield: $1.53 \mathrm{~g}(59 \%)$, blue solid. $\mathrm{R}_{\mathrm{f}}=0.68$ (toluene/ethyl acetate 15:1). UV/VIS: $\lambda_{\max }(\lg \varepsilon)=642 \mathrm{~nm}(4.11)$ in dichloromethane. El/MS $(70 \mathrm{eV}): \mathrm{m} / \mathrm{z}(\%)=422(67)$ $\left[\left({ }^{37} \mathrm{Cl}\right) \mathrm{M}\right]^{+}, 420(100)\left[\left({ }^{35} \mathrm{Cl}\right) \mathrm{M}\right]^{+}$(calcd for $\left.\mathrm{C}_{23} \mathrm{H}_{14} \mathrm{Cl}_{2} \mathrm{~N}_{2} \mathrm{O}_{2}, 420.04\right) .{ }^{1} \mathrm{H}-\mathrm{NMR}\left(360 \mathrm{MHz},\left[\mathrm{D}_{6}\right]-\mathrm{DMSO}\right): \delta=7.69$ (d, $\left.1 \mathrm{H}, J_{4,6}=2.0 \mathrm{~Hz}, \mathrm{H}-4\right), 7.62\left(\mathrm{dd}, 1 \mathrm{H}, J_{4,6}=2.0 \mathrm{~Hz}, J_{6,7}=8.7 \mathrm{~Hz}, \mathrm{H}-6\right), 7.55\left(\mathrm{~d}, 1 \mathrm{H}, J_{4^{\prime}, 6^{\prime}}=2.0 \mathrm{~Hz}, \mathrm{H}-4^{\prime}\right), 7.54(\mathrm{~d}, 1 \mathrm{H}$, $\left.J_{4^{\prime}, 6^{\prime}}=2.0 \mathrm{~Hz}, J_{6^{\prime}, 7^{\prime}}=8.7 \mathrm{~Hz}, \mathrm{H}-6^{\prime}\right), 7.39\left(\mathrm{~d}, 2 \mathrm{H}, J=8.7 \mathrm{~Hz}, \mathrm{H}-7, \mathrm{H}-7^{\prime}\right), 7.22-7.18\left(\mathrm{~m}, 3 \mathrm{H}, \mathrm{H}_{p^{-}}-\mathrm{Ar}, \mathrm{H}_{m}-\mathrm{Ar}\right), 7.08(\mathrm{~d}, 2 \mathrm{H}$, $J=7.2 \mathrm{~Hz}, \mathrm{H}_{o^{-}} \mathrm{Ar}$ ), $5.74\left(\mathrm{~s}, 2 \mathrm{H}, \mathrm{CH}_{2}\right) .{ }^{13} \mathrm{C}-\mathrm{NMR}$ (90 MHz, [D $]$-DMSO): $\delta=186.8\left(\mathrm{C}_{\mathrm{q}^{-}}-3\right), 184.4\left(\mathrm{C}_{\mathrm{q}^{-}} 3^{\prime}\right), 151.1\left(\mathrm{C}_{\mathrm{q}^{-}}\right.$ 7a'), 150.4 ( $\left.\mathrm{C}_{\mathrm{q}^{-}}-7 \mathrm{a}\right), 137.3$ ( $\left.\mathrm{C}_{\mathrm{i}}-\mathrm{Ar}\right), 135.6,135.4$ (C-6, C-6'), 128.6 ( $\left.\mathrm{C}_{o^{-}}-\mathrm{Ar}\right), 127.3\left(\mathrm{C}_{p^{-}}-\mathrm{Ar}\right), 126.7\left(\mathrm{C}_{m^{-}}-\mathrm{Ar}\right), 125.6\left(\mathrm{C}_{\mathrm{q}^{-}}\right.$ 5), $124.7\left(C_{q^{-}} 5^{\prime}\right), 124.6\left(C_{q^{-}}-2\right), 123.3\left(C_{q^{-}} 2^{\prime}\right), 123.1$ (C-4'), $122.7(C-4), 121.7\left(C_{q^{-}}-3 a^{\prime}\right), 120.1\left(C_{q}-3 a\right), 115.1(C-7)$, 114.1 (C-7'), $50.1\left(\mathrm{CH}_{2}\right)$.

N-[(4"-Azido-2",3"'-di-O-benzoyl-4",6"-dideoxy- $\beta$-D-glucopyranosyl)-N'-benzyl]-5,5'-dichloroindigo

(20). Imidate 10 (213 mg, $0.39 \mathrm{mmol}, 1.0 \mathrm{eq}), N$-benzyl-5,5'-dichloroindigo 11 (165 mg, $0.39 \mathrm{mmol}, 1.0$ eq) and molecular sieves ( $4 \AA, 400 \mathrm{mg}$ ) were suspended in absolute dichloromethane $(22 \mathrm{~mL})$, stirred for 30 minutes at room temperature followed by cooling to $-18{ }^{\circ} \mathrm{C}$. The reaction was started by adding TMSOTf $(23 \mu \mathrm{L}$, $0.127 \mathrm{mmol}, 0.33 \mathrm{eq})$. The reaction was stirred for $2 \mathrm{~h}$ at $-18^{\circ} \mathrm{C}$ and slowly warmed to room temperature over $12 \mathrm{~h}$. The reaction was stopped by adding saturated potassium hydrogen carbonate $(10 \mathrm{~mL})$. After 10 minutes the suspension was filtered through Celite and washed with dichloromethane. The filtrate was extracted with water, saturated potassium hydrogen carbonate and brine. The organic layer was dried $\left(\mathrm{MgSO}_{4}\right)$ and filtered. The filtrate was concentrated in vacuo and the residue purified by flash chromatography $(5 \times 18 \mathrm{~cm}$, eluent: cyclohexane/ethyl acetate $30: 1 \rightarrow 20: 1 \rightarrow 15: 1 \rightarrow 10: 1) .89$ mg (54\%) of N-benzyl-5,5'-dichloroindigo 11 were recovered. Yield: $139 \mathrm{mg} 20$ (44.6\%), turquoise solid. $R_{f}=0.33$ (cyclohexane/ethyl acetate 4:1). UV/VIS: $\lambda$ max $(\lg \varepsilon)=646 \mathrm{~nm}(4.04)$ in dichloromethane. $[\alpha]_{\mathrm{D}}^{23}=+5378.2^{\circ}(\mathrm{c}=5.8 \mu \mathrm{g} / \mathrm{mL}$, dichloromethane). ESI $/ \mathrm{MS}: \mathrm{m} / z=$ $800.39\left[\left({ }^{35 / 35} \mathrm{Cl}\right) \mathrm{M}+\mathrm{H}\right]^{+}, 802.39\left[\left({ }^{35 / 37} \mathrm{Cl}\right) \mathrm{M}+\mathrm{H}\right]^{+}, 804.39\left[\left({ }^{37 / 37} \mathrm{Cl}\right) \mathrm{M}+\mathrm{H}\right]^{+}$(calcd for $\left.\mathrm{C}_{43} \mathrm{H}_{32} \mathrm{Cl}_{2} \mathrm{~N}_{5} \mathrm{O}_{7}, 800.17\right) .{ }^{1} \mathrm{H}-\mathrm{NMR}$ (360 MHz, [D6]-DMSO): $\delta=7.82-7.78(\mathrm{~m}, 3 \mathrm{H}, \mathrm{H}-7, \mathrm{H}-4, \mathrm{H}-\mathrm{Ar}), 7.73\left(\mathrm{dd}, 1 \mathrm{H}, J_{4,6}=2.1 \mathrm{~Hz}, J_{6,7}=8.7 \mathrm{~Hz}, \mathrm{H}-6\right), 7.57$ (dd, $1 \mathrm{H}, J=7.5 \mathrm{~Hz}, \mathrm{H}-\mathrm{Ar}$ ), $7.52\left(\mathrm{~d}, 1 \mathrm{H}, J_{4^{\prime}, 6^{\prime}}=2.1 \mathrm{~Hz}, \mathrm{H}-4^{\prime}\right), 7.47$ (dd, $\left.1 \mathrm{H}, J_{4^{\prime}, 6^{\prime}}=2.1 \mathrm{~Hz}, J_{6^{\prime}, 7^{\prime}}=8.6 \mathrm{~Hz}, \mathrm{H}-6^{\prime}\right), 7.45-$ 
7.40 (m, 5H, H-Ar), 7.38-7.27 (m, 6H, H-Ar), 6.86 (dd, 2H, J = 7.8 Hz, H-Ar), 6.64 (d, 1H, J6', $\left.7^{\prime}=8.6 \mathrm{~Hz}, \mathrm{H}-7^{\prime}\right), 6.00$ (d, $\left.1 \mathrm{H}, J_{1^{\prime \prime}, 2^{\prime \prime}}=9.1 \mathrm{~Hz}, \mathrm{H}-1^{\prime \prime}\right), 5.89$ (dd, $\left.1 \mathrm{H}, J_{2^{\prime \prime}, 3^{\prime \prime}}=J_{3^{\prime \prime}, 4^{\prime \prime}}=9.3 \mathrm{~Hz}, \mathrm{H}-3^{\prime \prime}\right), 5.76$ (dd, $\left.1 \mathrm{H}, J_{1^{\prime \prime}, 2^{\prime \prime}}=J_{2^{\prime \prime}, 3^{\prime \prime}}=9.3 \mathrm{~Hz}, \mathrm{H}-2^{\prime \prime}\right)$,

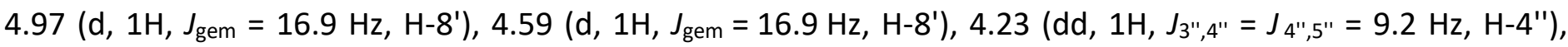
4.05-3.90 (m, 1H, H-5"), 1.59 (d, 3H, J" $\left.5^{\prime \prime} 6^{\prime \prime}=6.0 \mathrm{~Hz}, \mathrm{H}-6^{\prime \prime}\right) .{ }^{13} \mathrm{C}-\mathrm{NMR}$ (90 MHz, [D6]-DMSO): $\delta=185.6$ (Cq-3),

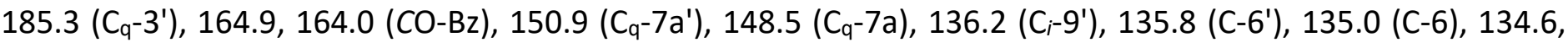
133.7, 133.8 ( $\left.C_{p}-A r\right), 129.5,129.1,128.8$ (C-Ar), 128.6, 128.4 ( $\left.C_{i}-A r\right), 128.3(C-A r), 127.6\left(C_{q}-5\right), 127.1\left(C_{q}-5 '\right)$,

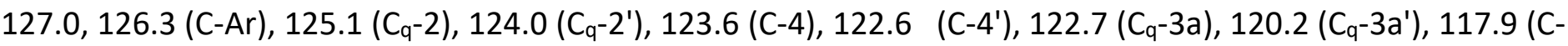
7), 115.2 (C-7'), 85.3 (C-1"), 74.4 (C-3'), 73.4 (C-5"), 69.1 (C-3"), 65.3 (C-4"), 54.5 (CH $\left.2-8^{\prime}\right), 18.2$ (C-6").

$\boldsymbol{N}$-(4"-Azido-2",3"-di-O-benzoyl-4",6"-dideoxy- $\beta$-D-glucopyranosyl)-5,5'-dichloroindigo (21). Glycoside 20 (184 mg, $0.23 \mathrm{mmol}, 1.0 \mathrm{eq})$ was dissolved in toluene/acetic acid (52 mL, 1:1) and stirred in an open flask for $28 \mathrm{~h}$ at $50{ }^{\circ} \mathrm{C}$. The solution was diluted with dichloromethane $(200 \mathrm{~mL})$, extracted with water and saturated potassium hydrogen carbonate. The organic layer was dried $\left(\mathrm{MgSO}_{4}\right)$ and filtered. The filtrate was concentrated in vacuo and the residue purified by flash chromatography $(5 \times 18 \mathrm{~cm}$, eluent: cyclohexane/ethyl acetate $20: 1 \rightarrow 15: 1)$. Yield: $98 \mathrm{mg}(60 \%)$, blue solid. $R_{f}=0.53$ (toluene). UV/VIS: $\lambda_{\max }(\lg \varepsilon)=618 \mathrm{~nm}(3.35) \mathrm{in}$ dichloromethane. $[\alpha]_{D}{ }^{23}=+4929.0^{\circ}\left(c=5.1 \mu \mathrm{g} / \mathrm{mL}\right.$, dichloromethane). ESI/MS: $m / z=709.99\left[\left({ }^{35 / 35} \mathrm{Cl}\right) \mathrm{M}+\mathrm{H}\right]^{+}$, $712.01\left[\left({ }^{35 / 37} \mathrm{Cl}\right) \mathrm{M}+\mathrm{H}\right]^{+}, 713.99\left[\left({ }^{37 / 37} \mathrm{Cl}\right) \mathrm{M}+\mathrm{H}\right]^{+}$(calcd for $\left.\mathrm{C}_{36} \mathrm{H}_{26} \mathrm{Cl}_{2} \mathrm{~N}_{5} \mathrm{O}_{7}, 710.12\right),{ }^{1} \mathrm{H} \mathrm{NMR}\left(270 \mathrm{MHz},\left[\mathrm{D}_{6}\right]-\right.$ DMSO): $\delta=10.91$ (s, 1H, NH), 7.84-7.80 (m, 3H, H-7, $\left.\mathrm{H}_{o}-\mathrm{Ar}\right)$, 7.77-7.71 (m, 2H, H-4, H-6), 7.64-7.56 (m, 3H, H-4', H-6', $\mathrm{H}_{p}-\mathrm{Ar}$ ), 7.53-7.43 (m, 5H, $\mathrm{H}_{o}-\mathrm{Ar}, \mathrm{H}_{p^{-}} \mathrm{Ar}$ ), 7.40 (d, 2H, J=8.6 Hz, H-7'), 7.29 (dd, 2H, J = 7.7 Hz, $\left.\mathrm{H}_{m^{-}}-\mathrm{Ar}\right), 6.38$ $\left(\mathrm{d}, 1 \mathrm{H}, J_{1^{\prime \prime}, 2^{\prime \prime}}=8.6 \mathrm{~Hz}, \mathrm{H}-1^{\prime \prime}\right), 5.75-5.65\left(\mathrm{~m}, 2 \mathrm{H}, \mathrm{H}-2^{\prime \prime}, \mathrm{H}-3^{\prime \prime}\right), 4.28\left(\mathrm{dd}, 1 \mathrm{H}, J_{3^{\prime \prime}, 4^{\prime \prime}}=J_{4^{\prime \prime}, 5^{\prime \prime}}=9.6 \mathrm{~Hz}, \mathrm{H}-4^{\prime \prime}\right), 4.02-3.90$

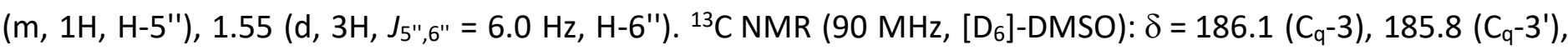

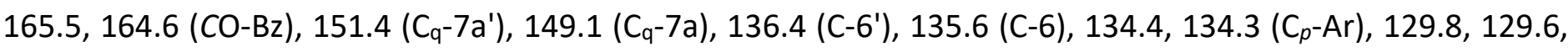
129.5, 129.4, 129.2 (C-Ar), 129.0 ( $\left.C_{i}-A r\right), 128.2\left(C_{q}-5\right), 127.7\left(C_{q^{-}} 5^{\prime}\right), 125.9\left(C_{q}-2\right), 125.6\left(C_{q}-2^{\prime}\right), 124.6\left(C_{q}-3 a\right)$,

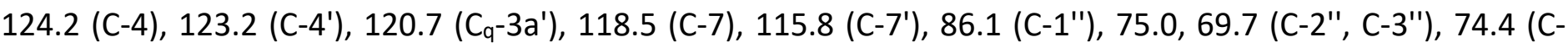
5"), 64.9 (C-4"), 18.7 (C-6").

O'-[(4"-Azido-2",3"-di-O-benzoyl-4",6"'-dideoxy- $\beta$-D-glucopyranosyl)- $N$ '-benzyl]-5,5'-dichloroindigo

(20a) and

O'-[(4"-azido-2",3"-di-O-benzoyl-4",6"-dideoxy- $\beta$-D-glucopyranosyl)- $N$ '-benzyl]-5,5'-dichloroindigo (20b). Imidate 10 (100 mg, $0.185 \mathrm{mmol}, 1.0 \mathrm{eq}$ ), $N$-benzyl-5,5'-dichloroindigo 11 (78 mg, $0.185 \mathrm{mmol}, 1.0 \mathrm{eq})$ and molecular sieves ( $4 \AA, 200 \mathrm{mg}$ ) were suspended in absolute dichloromethane $(11 \mathrm{~mL})$, stirred for 30 minutes at room temperature and subsequently cooled to $-18^{\circ} \mathrm{C}$. The reaction was started by addition of TMSOTf (11 $\mu \mathrm{L}$, $0.062 \mathrm{mmol}, 0.33 \mathrm{eq})$. After stirring for $2 \mathrm{~h}$ at $-18{ }^{\circ} \mathrm{C}$ the reaction was quenched by adding $\mathrm{Et}_{3} \mathrm{~N}$ in dichloromethane (200 $\mu \mathrm{L}, 0.5 \%)$. On TLC in cyclohexane/ethyl acetate 4:1 three colored spots were obtained: $R_{f}=0.64$ (blue), $R_{f}=0.51$ (green), $R_{f}=0.44$ (red). The reaction mixture was filtered over Celite and the filtrate was extracted with saturated sodium hydrogen carbonate solution. The organic layer was dried $\left(\mathrm{MgSO}_{4}\right)$ and filtered. After adding $\mathrm{Et}_{3} \mathrm{~N}$ in dichloromethane $(7 \mathrm{~mL}, 0.5 \%)$ the filtrate was concentrated in vacuo and the residue purified by flash chromatography $(2.5 \times 14 \mathrm{~cm}$, eluent: cyclohexane/ethyl acetate 30:1 containing $0.5 \%$ $\mathrm{Et}_{3} \mathrm{~N}$ ). Four fractions were obtained: fraction 1: indigo 11 (blue) $R_{f}=0.64$; fraction 2: $N$-glycoside 20 (green) $R_{f}$ $=0.51$ ); fraction 3: $\mathrm{N}$-glycoside 20 and $\alpha$-O-glycoside $20 \mathrm{~b}$ (red) $\mathrm{R}_{\mathrm{f}}=0.48$; fraction 4: $\beta$-O-glycoside $20 \mathrm{a}$ (red) $\mathrm{R}_{\mathrm{f}}$ $=0.44\left(R_{f}\right.$ values in cyclohexane/ethyl acetate 4:1).

$\beta-O-G l y c o s i d e ~(20 a)$. Yield: $6.6 \mathrm{mg}$ (4.5\%). UV/VIS: $\lambda_{\max }(\lg \varepsilon)=488 \mathrm{~nm}(2.41)$ in dichloromethane. $[\alpha]_{\mathrm{D}}^{23}=+$ $1579^{\circ}$ (c $=9.5 \mu \mathrm{g} / \mathrm{mL}$, dichloromethane). ESI/MS: $m / z=822.37\left[\left({ }^{35 / 35} \mathrm{Cl}\right) \mathrm{M}+\mathrm{Na}\right]^{+}, 824.36\left[\left({ }^{35 / 37} \mathrm{Cl}\right) \mathrm{M}+\mathrm{Na}\right]^{+}$, $826.34\left[\left({ }^{37 / 37} \mathrm{Cl}\right) \mathrm{M}+\mathrm{Na}\right]^{+}$(calcd for $\mathrm{C}_{43} \mathrm{H}_{31} \mathrm{Cl}_{2} \mathrm{~N}_{5} \mathrm{O}_{7} \mathrm{Na}$, (822.15). ${ }^{1} \mathrm{H}-\mathrm{NMR}\left(360 \mathrm{MHz},\left[\mathrm{D}_{6}\right]-\mathrm{DMSO}\right): \delta=7.86(\mathrm{~d}, 2 \mathrm{H}, \mathrm{J}$

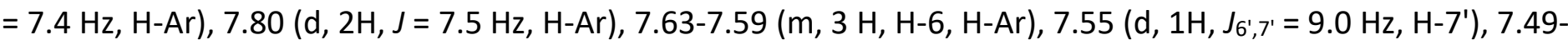
7.42 (m, 4H, H-Ar), 7.41 (bs, $\left.1 \mathrm{H}, \mathrm{H}-4^{\prime}\right), 7.38$ (d, 1H, $\left.J_{6,7}=7.1 \mathrm{~Hz}, \mathrm{H}-7\right), 7.35$ (d, $\left.1 \mathrm{H}, J_{4,6}=1.7 \mathrm{~Hz}, \mathrm{H}-4\right), 7.26$ (dd, 
$\left.1 \mathrm{H}, J_{6^{\prime}, 7^{\prime}}=8.9 \mathrm{~Hz}, J_{4^{\prime}, 6^{\prime}}=1.7 \mathrm{~Hz}, \mathrm{H}-6^{\prime}\right), 7.19-7.11(\mathrm{~m}, 3 \mathrm{H}, \mathrm{H}$-benzyl), 6.93 (d, 2H, J = 7.0 Hz, H-benzyl), $5.71(\mathrm{~d}, 1 \mathrm{H}$, $\left.J_{\text {gem }}=16.2 \mathrm{~Hz}, \mathrm{H}-8^{\prime} \mathrm{a}\right), 5.64$ (d, $\left.1 \mathrm{H}, J_{\text {gem }}=16.2 \mathrm{~Hz}, \mathrm{H}-8^{\prime} \mathrm{b}\right), 5.62$ (dd, $\left.1 \mathrm{H}, J_{2^{\prime \prime}, 3^{\prime \prime}}=J_{3^{\prime \prime}, 4^{\prime \prime}}=9.3 \mathrm{~Hz}, \mathrm{H}-3^{\prime \prime}\right), 5.38$ (dd, $1 \mathrm{H}$, $\left.J_{1^{\prime \prime}, 2^{\prime \prime}}=J_{2^{\prime \prime}, 3^{\prime \prime}}=9.3 \mathrm{~Hz}, \mathrm{H}-2^{\prime \prime}\right), 5.29$ (d, $\left.1 \mathrm{H}, J_{1^{\prime \prime}, 2^{\prime \prime}}=8.0 \mathrm{~Hz}, \mathrm{H}-1^{\prime \prime}\right), 3.96$ (dd, $\left.1 \mathrm{H}, J_{3^{\prime \prime}, 4^{\prime \prime}}=J_{4^{\prime \prime}, 5^{\prime \prime}}=9.3 \mathrm{~Hz}, \mathrm{H}-4^{\prime \prime}\right), 3.67$ (td, $\left.1 \mathrm{H}, J_{4^{\prime \prime}, 5^{\prime \prime}}=9.3, J_{5^{\prime \prime}, 6^{\prime \prime}}=6.1 \mathrm{~Hz} \mathrm{H}-5^{\prime \prime}\right), 0.99$ (d, 3H, $\left.J_{5^{\prime \prime}, 6^{\prime \prime}}=6.1 \mathrm{~Hz}, \mathrm{H}-6^{\prime \prime}\right) .{ }^{13} \mathrm{C}-\mathrm{NMR}\left(90 \mathrm{MHz},\left[\mathrm{D}_{6}\right]-\mathrm{DMSO}\right): \delta=187.8$

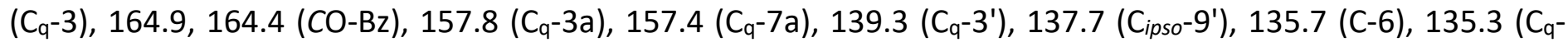
7a'), 133.9, 133.8 ( $\left.C_{p}-A r\right), 133.1$ ( $\left.C_{q}-2\right), 129.3,129.0,128.9$ (C-Ar), 128.5 ( $C_{m}$-benzyl), 128.4, $128.3\left(C_{q}-A r\right), 127.2$ ( $C_{p}$-benzyl), 126.4 ( $C_{o}$-benzyl),125.6 (C-6'), 125.3 ( $\left.C_{q^{-}}-3 a^{\prime}\right), 124.5$ (C-4), 123.3 (C-7), $123.0\left(C_{q^{-}}-5\right), 120.9$ (C $\left.q^{-}-2^{\prime}\right)$, $120.5\left(\mathrm{C}_{q^{-}} 5^{\prime}\right), 117.8$ (C-4'), 113.4 (C-7'), 101.4 (C-1'), 73.4 (C-3'), $72.0\left(\mathrm{C}-2^{\prime \prime}\right), 69.7$ (C-5'), $63.9\left(\mathrm{C}-4^{\prime \prime}\right), 47.2\left(\mathrm{CH}_{2-}\right.$ $\left.8^{\prime}\right), 17.2$ (C-6").

$\alpha$-O-Glycoside (20b). The $\alpha$-O-glycoside $20 \mathrm{~b}$ was isolated from fraction 3 by flash chromatography $(5 \times 17 \mathrm{~cm}$, eluent: cyclohexane/ethyl acetate 30:1+0.5\% NEt $\left.\rightarrow 25: 1+0.5 \% \mathrm{NEt}_{3}\right)$. Yield: $2.4 \mathrm{mg}(1.6 \%)$. ESI/MS: $\mathrm{m} / \mathrm{z}=$ $800.93\left[\left({ }^{35 / 35} \mathrm{Cl}\right) \mathrm{M}+\mathrm{H}\right]^{+}, 802.93\left[\left({ }^{35 / 37} \mathrm{Cl}\right) \mathrm{M}+\mathrm{H}\right]^{+}, 804.93\left[\left({ }^{37 / 37} \mathrm{Cl}\right) \mathrm{M}+\mathrm{H}\right]^{+}$(calcd for $\left.\mathrm{C}_{43} \mathrm{H}_{32} \mathrm{Cl}_{2} \mathrm{~N}_{5} \mathrm{O}_{7}, 800.17\right) .{ }^{1} \mathrm{H} \mathrm{NMR}$ (360 MHz, [D $\mathrm{D}_{6}$-DMSO): $\delta=7.93$ (d, 2H, J = 7.3 Hz, H-Ar), 7.71 (dd, $1 \mathrm{H}, J_{4.6}=1.9 \mathrm{~Hz}, J_{6,7}=8.2 \mathrm{~Hz}, \mathrm{H}-6$ ), 7.67 (d, $2 \mathrm{H}, J=7.4 \mathrm{~Hz}, \mathrm{H}-\mathrm{Ar}), 7.61\left(\mathrm{~d}, 1 \mathrm{H}, J_{4^{\prime}, 6^{\prime}}=2.0 \mathrm{~Hz}, \mathrm{H}-4^{\prime}\right), 7.59\left(\mathrm{~d}, 1 \mathrm{H},{ }^{4} J_{4,6}=1.9 \mathrm{~Hz}, \mathrm{H}-4\right), 7.56-7.46\left(\mathrm{~m}, 4 \mathrm{H}, \mathrm{H}-7^{\prime}, \mathrm{H}-\right.$ Ar), $7.44\left(\mathrm{~d}, 1 \mathrm{H}, \mathrm{dd}, 1 \mathrm{H}, J_{6,7}=8.1 \mathrm{~Hz}, \mathrm{H}-7\right), 7.28\left(\mathrm{dd}, 2 \mathrm{H}, J=7.7 \mathrm{~Hz}, \mathrm{H}-\mathrm{Ar}\right.$ ), 7.25 (dd, $1 \mathrm{H}, J_{6^{\prime}, 7^{\prime}}=2.0 \mathrm{~Hz}, J=6.6 \mathrm{~Hz}$

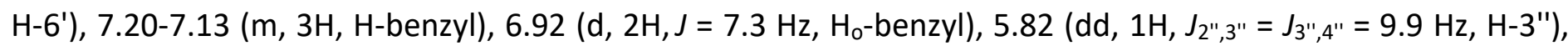
$5.81\left(\mathrm{~d}, 1 \mathrm{H}, J_{1^{\prime \prime}, 2^{\prime \prime}}=3.4 \mathrm{~Hz}, \mathrm{H}-1^{\prime \prime}\right), 5.66\left(\mathrm{~d}, 1 \mathrm{H}, J_{\text {gem }}=16.5 \mathrm{~Hz}, \mathrm{H}-8^{\prime}\right), 5.58\left(\mathrm{~d}, 1 \mathrm{H}, J_{\text {gem }}=16.5 \mathrm{~Hz}, \mathrm{H}-8^{\prime}\right), 5.35$ (dd, $\left.1 \mathrm{H}, J_{1^{\prime \prime}, 2^{\prime \prime}}=3.4 \mathrm{~Hz}, J_{2^{\prime \prime}, 3^{\prime \prime}}=9.9 \mathrm{~Hz}, \mathrm{H}-2^{\prime \prime}\right), 4.13\left(\mathrm{dd}, 1 \mathrm{H}, J_{3^{\prime \prime}, 4^{\prime \prime}}=J_{4^{\prime \prime}, 5^{\prime \prime}}=9.9 \mathrm{~Hz}, \mathrm{H}-4^{\prime \prime}\right), 4.05\left(\mathrm{td}, 1 \mathrm{H}, J_{4^{\prime \prime}, 5^{\prime \prime}}=9.9 \mathrm{~Hz}\right.$, $\left.J_{5^{\prime \prime}, 6^{\prime \prime}}=5.9 \mathrm{~Hz}, \mathrm{H}-5^{\prime \prime}\right), 1.19$ (d, 3H, $\left.J_{5^{\prime \prime}, 6^{\prime \prime}}=5.9 \mathrm{~Hz}, \mathrm{H}-6^{\prime \prime}\right) .{ }^{13} \mathrm{C} \mathrm{NMR}\left(90 \mathrm{MHz},\left[\mathrm{D}_{6}\right]-\mathrm{DMSO}\right): \delta=188.7\left(\mathrm{C}_{\mathrm{q}}-3\right), 165.1$, 164.9 (CO-Bz), 157.5 ( $\left.C_{q^{-}}-3 a\right), 157.0\left(C_{q^{-}}-7 a\right), 140.2$ ( $\left.C_{q^{-}}-3^{\prime}\right), 137.7\left(C_{i}-9 '\right), 136.1(C-6), 135.4\left(C_{q^{-}}-7 a^{\prime}\right), 133.9,133.8$ $\left(C_{p}-A r\right), 133.2\left(C_{q}-2\right), 129.3,129.1,128.9,128.7$ (C-Ar), $128.6\left(C_{q} A r\right), 128.4\left(C_{m}\right.$-benzyl), $128.1\left(C_{q}-A r\right), 127.3\left(C_{p^{-}}\right.$ benzyl), 126.5 ( $\mathrm{C}_{\mathrm{o}}$-benzyl), 125.7 (C-6'), 125.3 ( $\left.\mathrm{C}_{\mathrm{q}} 3 \mathrm{a}^{\prime}\right), 124.9$ (C-4), 123.5 (C-7), $123.1\left(\mathrm{C}_{\mathrm{q}}-5\right), 119.8\left(\mathrm{C}_{\mathrm{q}}-2^{\prime}\right), 119.6$ $\left(C_{q^{-}} 5^{\prime}\right), 117.9\left(C-4^{\prime}\right), 113.6\left(C^{\prime}-7^{\prime}\right), 99.3$ (C-1'), 71.5 (C-2'), $70.7\left(C-3^{\prime \prime}\right), 67.8$ (C-5'), $63.9\left(C-4^{\prime \prime}\right), 47.6\left(C_{2}-8^{\prime}\right)$, 17.6 (C-6").

Akashin A (1). Under an argon atmosphere 21 was dissolved in thoroughly degassed and dried methanol (0.8 $\mathrm{mL} / \mathrm{mg}$ starting material) followed by addition of propanedithiol (30 eq) and diisopropylethylamine (15 eq). Upon addition of the amine the color of the solution changed from blue to pale yellow. After 3-4 $d$ at room temperature under strict exclusion of light the reaction was transferred into dichloromethane/acetic acid (99:1, $2 \mathrm{~mL} / \mathrm{mg}$ starting material) and stirred in an open flask for 60-90 minutes. Within this time the yellowish vat indigo was reoxidised to the desired blue indigo. The organic phase was extracted with hydrochloric acid (25 mM). The blue aqueous phase was submitted to solid phase extraction using SepPak- $\mathrm{C}_{18}$ material. Elution was carried out with acetonitrile in water ( $1 \%$ acetic acid) in steps of $2.5 \%$ from $0-30 \%$ acetonitrile. The product eluted from 17.5-25\% acetonitrile and was lyophilized. For removal of the acetic acid the product was chromatographed by a Sephadex LH 20 column using dichloromethane/methanol (6:4) for elution. Yield: 39$73 \%$ of 1 , blue solid. $R_{f}=0.10$ (dichloromethane/methanol 15:1). UV/VIS: $\lambda_{\max }(\lg \varepsilon)=620 \mathrm{~nm}(4.15) \mathrm{in}$ methanol. $[\alpha]_{D^{24}}=+2591.0^{\circ}\left(\mathrm{c}=6.9 \mu \mathrm{g} / \mathrm{mL}\right.$, methanol). ESI/MS: $m / z=476.12\left[\left({ }^{35 / 35} \mathrm{Cl}\right) \mathrm{M}+\mathrm{H}\right]^{+}, 478.14$ $\left[\left({ }^{35 / 37} \mathrm{Cl}\right) \mathrm{M}+\mathrm{H}\right]^{+}, \quad 480.13 \quad\left[\left({ }^{37 / 37} \mathrm{Cl}\right) \mathrm{M}+\mathrm{H}\right]^{+}$(calcd for $\mathrm{C}_{22} \mathrm{H}_{20} \mathrm{Cl}_{2} \mathrm{~N}_{3} \mathrm{O}_{5}, \quad$ (476.08). ${ }^{1} \mathrm{H}-\mathrm{NMR}$ (360 $\mathrm{MHz},\left[\mathrm{D}_{6}\right]-$ DMSO): $\delta=11.00$ (bs, $1 \mathrm{H}, \mathrm{N}^{\prime} H$ ), $7.71\left(\mathrm{~d}, 1 \mathrm{H}, J_{4,6}=1.4 \mathrm{~Hz}, \mathrm{H}-4\right), 7.64$ (dd, $\left.1 \mathrm{H}, J_{4,6}=1.4 \mathrm{~Hz}, J_{6,7}=9.0 \mathrm{~Hz}, \mathrm{H}-6\right), 7.63$ $\left(\mathrm{d}, 1 \mathrm{H}, J_{4^{\prime}, 6^{\prime}}=1.6 \mathrm{~Hz}, \mathrm{H}-4^{\prime}\right), 7.55\left(\mathrm{dd}, 1 \mathrm{H}, J_{4^{\prime}, 6^{\prime}}=1.6 \mathrm{~Hz}, J_{6^{\prime}, 7^{\prime}}=8.6 \mathrm{~Hz}, \mathrm{H}-6^{\prime}\right), 7.50\left(\mathrm{~d}, 1 \mathrm{H}, J_{6,7}=9.0 \mathrm{~Hz}, \mathrm{H}-7\right), 7.41(\mathrm{~d}$,

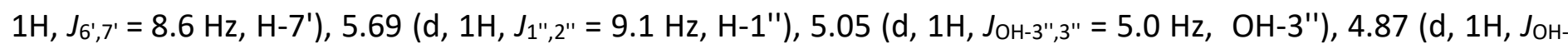

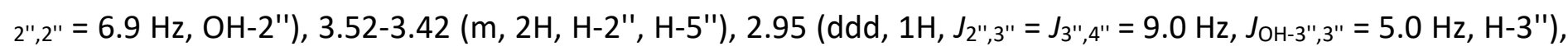
$2.31\left(\mathrm{dd}, 1 \mathrm{H}, J_{3^{\prime \prime}, 4^{\prime \prime}}=J_{4^{\prime \prime}, 5^{\prime \prime}}=9.0 \mathrm{~Hz}, \mathrm{H}-4^{\prime \prime}\right), 1.34\left(\mathrm{~d}, 3 \mathrm{H}, J_{5^{\prime \prime}, 6^{\prime \prime}}=5.8 \mathrm{~Hz}, \mathrm{H}-6^{\prime \prime}\right) .{ }^{13} \mathrm{C}-\mathrm{NMR}\left(90 \mathrm{MHz},\left[\mathrm{D}_{6}\right]-\right.$ DMSO): $\delta=185.2\left(\mathrm{C}_{q^{-}} 3^{\prime}\right), 184.1\left(\mathrm{C}_{\mathrm{q}^{-}}-3\right), 150.7$ ( $\left.\mathrm{C}_{\mathrm{q}^{-}}-7 \mathrm{a}^{\prime}\right), 149.2\left(\mathrm{C}_{\mathrm{q}}-7 \mathrm{a}\right), 135.6\left(\mathrm{C}-6^{\prime}\right), 134.7(\mathrm{C}-6), 126.6\left(\mathrm{C}_{\mathrm{q}}-5\right)$, $124.6\left(C_{q^{-}} 5^{\prime}\right), 124.2\left(C_{q}-2\right), 124.0\left(C_{q^{-}} 2^{\prime}\right), 123.9\left(C_{q^{-}}-3 a\right), 123.2\left(C-4{ }^{\prime}\right), 122.6(C-4), 120.2\left(C_{q^{-3}} a^{\prime}\right), 117.9(C-7)$, 
115.1 (C-7'), 88.0 (C-1"), 77.2 (C-3"), 76.3 (C-5"), 70.0 (C-2'), 59.2 (C-4"), 18.5 (C-6"). ${ }^{1} \mathrm{H}$ NMR (360 MHz, [D6]DMSO acidified with $\mathrm{HCl}$ ): $\delta=8.46-8.38$ (bs, $\left.1 \mathrm{H}, \mathrm{N}^{\prime} \mathrm{H}_{3}{ }^{+}\right), 7.71(\mathrm{bs}, 1 \mathrm{H}, \mathrm{H}-4), 7.61(\mathrm{~m}, 1 \mathrm{H}, \mathrm{H}-6), 7.61\left(\mathrm{~m}, 1 \mathrm{H}, \mathrm{H}-4{ }^{\prime}\right)$, $7.56\left(\mathrm{dd}, 1 \mathrm{H}, J_{4^{\prime}, 6^{\prime}}=1.7 \mathrm{~Hz}, J_{6^{\prime}, 7^{\prime}}=8.1 \mathrm{~Hz}, \mathrm{H}-6^{\prime}\right), 7.57-7.50(\mathrm{~m}, 1 \mathrm{H}, \mathrm{H}-7), 7.39\left(\mathrm{~d}, 1 \mathrm{H}, J_{6^{\prime}, 7^{\prime}}=8.5 \mathrm{~Hz}, \mathrm{H}-7^{\prime}\right), 5.74-5.69$ $\left(\mathrm{m}, 1 \mathrm{H}, \mathrm{H}-1^{\prime \prime}\right), 3.61$ (dd, $\left.1 \mathrm{H}, J_{1^{\prime \prime}, 2^{\prime \prime}}=J_{2^{\prime \prime}, 3^{\prime \prime}}=9.0 \mathrm{~Hz}, \mathrm{H}-2^{\prime \prime}\right), 2.95\left(\mathrm{dd}, 1 \mathrm{H}, J_{2 ", 3 "}=J_{3^{\prime \prime}, 4^{\prime \prime}}=9.0 \mathrm{~Hz}, \mathrm{H}-3^{\prime \prime}\right), 3.00-2.86(\mathrm{~m}$,

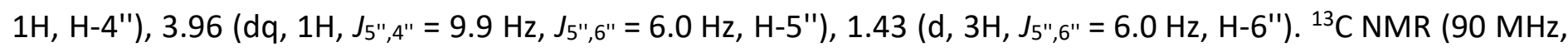
$\left[\mathrm{D}_{6}\right]$-DMSO acidified with $\mathrm{HCl} ;{ }^{13} \mathrm{C}$-signals taken from HMQC-COSY): $\delta=135.5$ (C-6'), 134.0 (C-6), 123.4 (C-4'), 122.1 (C-4), 117.1 (C-7), 114.5 (C-7'), 87.1 (C-1"), 72.6 (C-3"), 71.5 (C-5"), 69.4 (C-2"), 57.0 (C-4"), 18.2 (C-6").

Akashin B (2). Akashin A 1 (9.6 mg, 20 mol, 1.0 eq) was dissolved in methanol (9.6 mL). Acetic anhydride (192 $\mu \mathrm{L}, 2.0 \mathrm{mmol}, 100 \mathrm{eq})$ and water $(288 \mu \mathrm{L}, 16 \mathrm{mmol}, 800 \mathrm{eq})$ were added and mixture was stirred for 30 minutes at room temperature. The crude product was concentrated in vacuo after addition of toluene. The remainder was purified by solid phase extraction over SepPak- $\mathrm{C}_{18}$ material using a step gradient of acetonitrile in water (20-100\% in 10\%-steps). The product eluted from $40-70 \%$ acetonitrile and was lyophilized. Yield: 10.3 mg 2 (99.4\%), blue solid. $R_{f}=0.24$ (dichloromethane/methanol 15:1). UV/VIS: $\lambda_{\max }(\lg \varepsilon)=620 \mathrm{~nm}(4.18)$ in methanol. $[\alpha]_{D^{27}}=+4137.9^{\circ}\left(c=5.8 \mu \mathrm{g} / \mathrm{mL}\right.$, methanol). ESI/MS: $m / z=518.20\left[\left({ }^{35 / 35} \mathrm{Cl}\right) \mathrm{M}+\mathrm{H}\right]^{+}, 520.19$ $\left[\left({ }^{35 / 37} \mathrm{Cl}\right) \mathrm{M}+\mathrm{H}\right]^{+}, \quad 522.19 \quad\left[\left({ }^{37 / 37} \mathrm{Cl}\right) \mathrm{M}+\mathrm{H}\right]^{+}$(calcd for $\left.\mathrm{C}_{24} \mathrm{H}_{22} \mathrm{Cl}_{2} \mathrm{~N}_{3} \mathrm{O}_{6}, \quad 518.09\right) .{ }^{1} \mathrm{H}-\mathrm{NMR} \quad\left(360 \mathrm{MHz},\left[\mathrm{D}_{6}\right]-\right.$ DMSO): $\delta=11.01\left(\mathrm{~s}, 1 \mathrm{H}, \mathrm{N} H^{\prime}\right), 7.77\left(\mathrm{~d}, 1 \mathrm{H}, J_{\mathrm{NH}} \mathrm{H}^{\prime \prime}=8.9 \mathrm{~Hz}, \mathrm{NH} H^{\prime \prime}\right), 7.72(\mathrm{~s}, 1 \mathrm{H}, \mathrm{H}-4), 7.67\left(\mathrm{~d}, 1 \mathrm{H}, J_{6,7}=8.2 \mathrm{~Hz}, \mathrm{H}-\right.$ 6), 7.62 (s, $\left.1 \mathrm{H}, \mathrm{H}-4^{\prime}\right), 7.60-7.54\left(\mathrm{~m}, 2 \mathrm{H}, \mathrm{H}-6^{\prime}, \mathrm{H}-7\right), 7.41$ (d, $\left.1 \mathrm{H}, J_{6^{\prime}, 7^{\prime}}=8.2 \mathrm{~Hz}, \mathrm{H}-7^{\prime}\right), 5.71\left(\mathrm{~d}, 1 \mathrm{H}, J_{1^{\prime \prime}, 2^{\prime \prime}}=9.0 \mathrm{~Hz}, \mathrm{H}-\right.$ $1^{\prime \prime)}$, 5.0-4.95 (m, 2H, OH-2", OH-3"), 3.68-3.59 (m, 1H, H-5"), 3.60-3.49 (m, 2H, H-2", H-4"), 3.24 (ddd, $1 \mathrm{H}, \mathrm{J}^{\prime \prime}, 3^{\prime \prime}$ $\left.=J_{3^{\prime \prime}, 4^{\prime \prime}}=9.1 \mathrm{~Hz}, J_{\mathrm{OH}-3^{\prime \prime}, 3^{\prime \prime}}=5.9 \mathrm{~Hz}, \mathrm{H}-3^{\prime \prime}\right), 1.84$ (s, 3H, NH-Ac), 1.23 (d, 3H, J" J",6" = $\left.5.1 \mathrm{~Hz}, \mathrm{H}-6^{\prime \prime}\right) .{ }^{13} \mathrm{C}-\mathrm{NMR}(90 \mathrm{MHz}$, [D6]-DMSO): $\delta=187.4\left(\mathrm{C}_{\mathrm{q}^{-}}-3\right), 186.0\left(\mathrm{C}_{\mathrm{q}^{-}} 3^{\prime}\right), 170.1$ (CO-Ac), 151.5 ( $\mathrm{C}_{\mathrm{q}^{-}}$-7a'), $149.9\left(\mathrm{C}_{\mathrm{q}}-7 \mathrm{a}\right), 136.4$ (C-6'), 135.6 (C6), $127.1\left(C_{q^{-}}-5\right), 125.4\left(C_{q^{-}} 5^{\prime}\right), 125.0\left(C_{q^{-}} 2^{\prime}\right), 124.8\left(C_{q^{-}}-2\right), 124.7\left(C_{q^{-}}-3 a\right), 120.9\left(C_{q^{-}}-3 a^{\prime}\right), 123.9\left(C-4{ }^{\prime}\right), 123.4(C-4)$, 118.7 (C-7), 115.9 (C-7'), 88.6 (C-1"), 75.1 (C-3", C-5"), 71.0 (C-2"), 59.2 (C-4"), 23.8 (NAc), 19.1 (C-6").

Akashin C (3). Akashin A 1 (4 mg, $8.4 \mu \mathrm{mol}, 1.0 \mathrm{eq})$ was dissolved in absolute methanol (4.3 mL). Diacetyl (4.4 $\mu \mathrm{L}, 50.3 \mu \mathrm{mol}, 6.0 \mathrm{eq})$ in absolute methanol (200 $\mu \mathrm{L})$, trimethylorthoformate $(12 \mu \mathrm{L}, 110 \mu \mathrm{mol}, 13 \mathrm{eq})$ in absolute methanol $(200 \mu \mathrm{L})$ and camphorsulfonic acid $(0.39 \mathrm{mg}, 1.7 \mu \mathrm{mol}, 0.2 \mathrm{eq})$ in absolute methanol (200 $\mu \mathrm{L})$ were added. After stirring for $2-3 \mathrm{~h}$ at room temperature sodium cyanoborhydride (5.3 $\mathrm{mg}, 84 \mu \mathrm{mol}, 10$ eq) in absolute methanol $(200 \mu \mathrm{L})$ was added. After 25 minutes the reaction was concentrated in vacuo and purified by flash chromatography $(2 \times 10 \mathrm{~cm}$, solvent: dichloromethane/methanol 40:1 $\rightarrow$ 30:1 $\rightarrow$ 25:1 containing $0.1 \%$ formic acid). Yield: $2.7 \mathrm{mg} 3$ (59\%), blue solid. $\mathrm{R}_{\mathrm{f}}=0.54$ (dichloromethane/methanol 15:1). UV/VIS: $\lambda_{\max }(\lg \varepsilon)=618 \mathrm{~nm}$ (3.71) in methanol. $[\alpha]_{D}{ }^{27}=+1143^{\circ}(\mathrm{c}=14 \mu \mathrm{g} / \mathrm{mL}$, methanol). ESI $/ \mathrm{MS}: \mathrm{m} / \mathrm{z}=$ $546.75\left[\left({ }^{35 / 35} \mathrm{Cl}\right) \mathrm{M}+\mathrm{H}\right]^{+}, 548.73\left[\left({ }^{35 / 37} \mathrm{Cl}\right) \mathrm{M}+\mathrm{H}\right]^{+}, 550.74\left[\left({ }^{37 / 37} \mathrm{Cl}\right) \mathrm{M}+\mathrm{H}\right]^{+}$(calcd for $\left.\mathrm{C}_{26} \mathrm{H}_{26} \mathrm{Cl}_{2} \mathrm{~N}_{3} \mathrm{O}_{6}, 546.12\right) .{ }^{1} \mathrm{H}-$ $\operatorname{NMR}\left(360 \mathrm{MHz}, \mathrm{CD}_{3} \mathrm{OD}\right): \delta=7.72(\mathrm{~s}, 1 \mathrm{H}, \mathrm{H}-4), 7.62-7.58\left(\mathrm{~m}, 3 \mathrm{H}, \mathrm{H}-4^{\prime}, \mathrm{H}-6, \mathrm{H}-7\right), 7.50\left(\mathrm{~d}, 1 \mathrm{H}, J_{6^{\prime}, 7^{\prime}}=8.7 \mathrm{~Hz}, \mathrm{H}-6^{\prime}\right)$, $7.22\left(\mathrm{~d}, 1 \mathrm{H}, J_{6^{\prime}, 7^{\prime}}=8.7 \mathrm{~Hz}, \mathrm{H}-7^{\prime}\right), 6.05\left(\mathrm{~d}, 1 \mathrm{H}, J_{1^{\prime \prime}, 2^{\prime \prime}}=9.3 \mathrm{~Hz}, \mathrm{H}-1^{\prime \prime}\right), 3.91-3.82\left(\mathrm{~m}, 2 \mathrm{H}, \mathrm{H}-2^{\prime \prime}, \mathrm{H}-5^{\prime \prime}\right), 3.77$ (dd, $1 \mathrm{H}$,

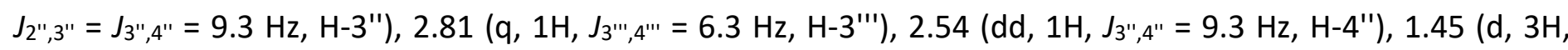

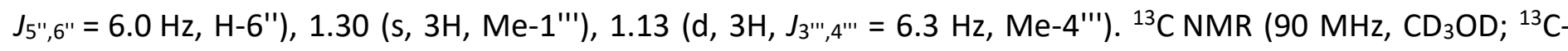
signals taken from HMQC-COSY): $\delta=135.0$ (C-6'), 133.9 (C-6), 122.7 (C-4'), 121.9 (C-4), 116.9 (C-7), 113.3 (C$\left.7^{\prime}\right), 87.7$ (C-1"), 73.7 (C-3", C-5"), 67.3 (C-2'), 61.6 (C-4"), 57.1 (C-3"'), 23.8 (C-1'"), 15.9 (C-6"), 14.7 (C-4'"'). ${ }^{1} \mathrm{H}-$ NMR (360 MHz, [D6]-DMSO): $\delta=11.00(\mathrm{~s}, 1 \mathrm{H}, \mathrm{NH}$ ), $7.72(\mathrm{~s}, 1 \mathrm{H}, \mathrm{H}-4), 7.65-7.60$ (m, 2H, H-4', H-6'), 7.59-7.51 (m, 2H, H-6, H-7), 7.40 (d, $\left.1 \mathrm{H}, J_{6^{\prime}, 7^{\prime}}=8.5 \mathrm{~Hz}, \mathrm{H}-7^{\prime}\right), 5.80$ (d, $\left.1 \mathrm{H}, J_{1^{\prime \prime}, 2^{\prime \prime}}=8.4 \mathrm{~Hz}, \mathrm{H}-1^{\prime \prime}\right), 5.67$ (s, 1H, OH-2'"'), 4.88 (d, $\left.1 \mathrm{H}, J_{\mathrm{OH}-2 ", 2 "}=5.0 \mathrm{~Hz}, \mathrm{OH}-2^{\prime \prime}\right), 3.73-3.46$ (m, 3H, H-2", H-4", H-5"), 2.65-2.57 (m, 1H, H-3"'), 2.45-2.40 (m, 1H, H-

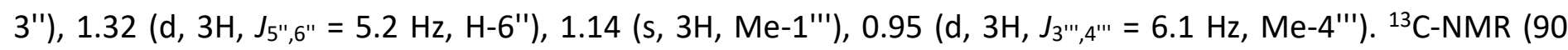
$\mathrm{MHz},\left[\mathrm{D}_{6}\right]-\mathrm{DMSO} ;{ }^{13} \mathrm{C}$-signals taken from HMQC-COSY): $\delta=135.5$ (C-6'), 134.5 (C-6), 122.9 (C-4'), 122.5 (C-4), 117.5 (C-7), 114.9 (C-7'), 88.1 (C-1"), 74.1 (C-2'), 73.8 (C-5"), 67.1 (C-4"), 61.9 (C-3"), 57.5 (C-3'"'), 25.3 (C-1"'), 17.8 (C-6"), 16.6 (C-4"'). 


\section{Acknowledgements}

We thank Prof. Hartmut Laatsch for providing refence spectra of akashin A-C.

\section{Supplementary Material}

LC-MS and ${ }^{1} \mathrm{H}$ NMR Spectra of compounds 4, 20, 20a, 20b, 1, 2, $\mathbf{3}$ are given in the supplementary material file associated with this paper.

\section{References}

1. Gribble, G. W.; Berthel, S. J. Studies in Natural Products Chemistry, 1993, 12 (Stereoselective Synthesis (Pt. H)), pp 365-409. https://doi.org/10.1016/B978-0-444-89366-6.70015-0

2. Faul, M. M.; Winneroski, L. L.; Krumrich, C. A. J. Org. Chem. 1998, 63 (17), 6053-6058. https://doi.org/10.1016/B978-0-444-89366-6.70015-0

3. Zhang, G.; Shen, J.; Cheng, H.; Zhu, L.; Fang, L.; Luo, S.; Muller, M. T.; Lee, G. E.; Wei, L.; Du, Y.; Sun, D.; Wang, P. G. J. Med. Chem. 2005, 48 (7), 2600-11. https://doi.org/10.1021/jm0493764

4. Moon, M. J.; Lee, S. K.; Lee, J. W.; Song, W. K.; Kim, S. W.; Kim, J. I.; Cho, C.; Choi, S. J.; Kim, Y. C. Bioorg. Med. Chem. 2006, 14 (1), 237-46. https://doi.org/10.1016/j.bmc.2005.08.008

5. Fuse, E.; Kuwabara, T.; Sparreboom, A.; Sausville, E. A.; Figg, W. D. J. Clin. Pharmacol. 2005, 45 (4), $394-$ 403. https://doi.org/10.1177/0091270005274549

6. Prudhomme, M. Anticancer Agents from Natural Products 2005, 499-517, 1 plate.

7. Wang, L.; Liu, X.; Chen, R. Natura and other derivatives of isoindigo, indigo, and indirubin for the treatment of cancer. 2001-21589 6566341, 20011213., 2003.

8. Wang, L.; Liu, X.; Chen, R. Derivatives of isoindigo, indigo and indirubin and use in treating cancer. 2002US39866 2003051900, 20021213., 2003.

9. Libnow, S.; Hein, M.; Michalik, D.; Langer, P. Tetrahedron Lett. 2006, 47 (38), 6907-6909. https://doi.org/10.1016/j.tetlet.2006.07.024

10. Libnow, S.; Methling, K.; Hein, M.; Michalik, D.; Harms, M.; Wende, K.; Flemming, A.; Koeckerling, M.; Reinke, H.; Bednarski, P. J.; Lalk, M.; Langer, P. Bioorg. Med. Chem. 2008, 16 (10), 5570-5583. https://doi.org/10.1016/j.bmc.2008.04.003

11. Maskey, R. P.; Grun-Wollny, I.; Fiebig, H. H.; Laatsch, H. Angew. Chem. Int. Ed. 2002, 41 (4), 597-599. https://doi.org/10.1002/1521-3773(20020215)41:4<597::AID-ANIE597>3.0.CO;2-Z

12. Maskey, R. P.; Grun-Wollny, I.; Laatsch, H. Nat. Prod. Res. 2005, 19 (2), 137-42. https://doi.org/10.1080/14786410410001704741

13. Braesel, J.; Clark, C. M.; Kunstman, K. J.; Green, S. J.; Maienschein-Cline, M.; Murphy, B. T.; Eustaquio, A. S. Microbiol. Resour. Announc. 2019, 8 (19). 
14. Stevens, C. L.; Blumbergs, P.; Daniher, F. A.; Otterbach, D. H.; Taylor, K. G. J. Org. Chem. 1966, 31 (9), $2822-$ 9. https://doi.org/10.1021/jo01347a020

15. Daubenspeck, J. M.; Zeng, H.; Chen, P.; Dong, S.; Steichen, C. T.; Krishna, N. R.; Pritchard, D. G.; Turnbough, C. L., Jr. J. Biol. Chem. 2004, 279 (30), 30945-53.

https://doi.org/10.1074/jbc.M401613200

16. Arai, T.; Takamizawa, Y. J. Antibiot. (Tokyo) 1954, 7 (5), 165-8.

17. Evans, J. R.; Weare, G. J. Antibiot. (Tokyo) 1977, 30 (7), 604-6. https://doi.org/10.7164/antibiotics.30.604

18. Miyaki, T.; Numata, K.; Nishiyama, Y.; Tenmyo, O.; Hatori, M.; Imanishi, H.; Konishi, M.; Kawaguchi, H. J. Antibiot. (Tokyo) 1981, 34 (6), 665-74. https://doi.org/10.7164/antibiotics.34.665

19. Lee, M. D.; Manning, J. K.; Williams, D. R.; Kuck, N. A.; Testa, R. T.; Borders, D. B. J. Antibiot. (Tokyo) 1989, 42 (7), 1070-87.

https://doi.org/10.7164/antibiotics.42.1070

20. Hein, M.; Michalik, D.; Langer, P. Synthesis 2005, (20), 3531-3534.

https://doi.org/10.1055/s-2005-918489

21. Hein, M.; Phuong, N. T. B.; Michalik, D.; Goerls, H.; Lalk, M.; Langer, P. Tetrahedron Lett. 2006, 47 (32), 5741-5745.

https://doi.org/10.1016/j.tetlet.2006.06.021

22. Kunz, H.; Harreus, A. Liebigs Ann. Chem. 1982, (1), 41-8.

https://doi.org/10.1002/jlac.198219820105

23. Schnabel, M.; Römpp, B.; Ruckdeschel, D.; Unverzagt, C. Tetrahedron Lett. 2004, 45 (2), 295-297. https://doi.org/10.1016/i.tetlet.2003.10.190

24. Schmidt, R. R. Angew. Chem. 1986, 98 (3), 213-36. https://doi.org/10.1002/ange.19860980305

25. Helferich, B.; Zirner, J. Ber. 1962, 95, 2604-11. https://doi.org/10.1002/cber.19620951103

26. Gros, E. G.; Deulofeu, V. J. Org. Chem. 1964, 29 (12), 3647-54. https://doi.org/10.1021/jo01035a054

27. Adak, A.; Mukhopadhyay, B. Carbohydr. Res. 2019, 476, 1-7.

28. Sadler, P. W.; Warren, R. L. J. Am. Chem. Soc. 1956, 78, 1251-5. https://doi.org/10.1021/ja01587a047

29. Russell, G. A.; Kaupp, G. J. Amer. Chem. Soc. 1969, 91 (14), 3851-9. https://doi.org/10.1021/ja01042a028

30. Holt, S. J.; Sadler, P. W. Proceedings of the Royal Society of London, Series B: Biological Sciences 1958, 148, 481-94. https://doi.org/10.1098/rspb.1958.0040

31. Voss, G.; Schramm, W. Helvetica Chimica Acta 2000, 83 (11), 2884-2892. https://doi.org/10.1002/1522-2675(20001108)83:11<2884::AID-HLCA2884>3.0.CO;2-C

32. Kessler, H.; Gehrke, M.; Griesinger, C. Angew. Chem. Int. Ed. Engl. 1988, 27 (4), 490-536. https://doi.org/10.1002/anie.198804901

33. Bayley, H.; Standring, D. N.; Knowles, J. R. Tetrahedron Lett. 1978, (39), 3633-4. https://doi.org/10.1016/S0040-4039(01)95015-4 
34. Baker, B. R.; Schaub, R. E. J. Org. Chem. 1954, 19, 646-60. https://doi.org/10.1021/jo01369a026

35. Ley, S. V.; Owen, D. R.; Wesson, K. E. J. Chem. Soc., Perkin Trans. 1 1997, (19), 2805-2806. https://doi.org/10.1039/a705360f

This paper is an open access article distributed under the terms of the Creative Commons Attribution (CC BY) license (http://creativecommons.org/licenses/by/4.0/) 